\title{
Infants with asthma : towards a multidisciplinary education protocol for primary care
}

Citation for published version (APA):

Mesters, E. P. E. (1993). Infants with asthma : towards a multidisciplinary education protocol for primary care. [Doctoral Thesis, Maastricht University]. Datawyse / Universitaire Pers Maastricht. https://doi.org/10.26481/dis.19930625em

Document status and date:

Published: 01/01/1993

DOI:

10.26481/dis.19930625em

Document Version:

Publisher's PDF, also known as Version of record

\section{Please check the document version of this publication:}

- A submitted manuscript is the version of the article upon submission and before peer-review. There can be important differences between the submitted version and the official published version of record.

People interested in the research are advised to contact the author for the final version of the publication, or visit the DOI to the publisher's website.

- The final author version and the galley proof are versions of the publication after peer review.

- The final published version features the final layout of the paper including the volume, issue and page numbers.

Link to publication

\footnotetext{
General rights rights.

- You may freely distribute the URL identifying the publication in the public portal. please follow below link for the End User Agreement:

www.umlib.nl/taverne-license

Take down policy

If you believe that this document breaches copyright please contact us at:

repository@maastrichtuniversity.nl

providing details and we will investigate your claim.
}

Copyright and moral rights for the publications made accessible in the public portal are retained by the authors and/or other copyright owners and it is a condition of accessing publications that users recognise and abide by the legal requirements associated with these

- Users may download and print one copy of any publication from the public portal for the purpose of private study or research.

- You may not further distribute the material or use it for any profit-making activity or commercial gain

If the publication is distributed under the terms of Article $25 \mathrm{fa}$ of the Dutch Copyright Act, indicated by the "Taverne" license above, 

Infants with asthma 
Financial support by the Netherlands Asthma Foundation: grants AF-86.25, AF-88.43 and AF-90.31.

CIP-GEGEVENS KONINKLIJKE BIBLIOTHEEK, DEN HAAG

Mesters, Elisabeth Pierre Eveline

Infants with asthma : towards a multicisciplinary

education protecol for primary care / Elisabeth Pierre

Eveline Mesters. - [S.I. : s.n.]. - III.

Thesis Maastricht. - With ref, - With summary in Dutch.

ISBN 90-9006145-2

Trefw.: astma ; kinderen.

Produktie: Datawyse Maastricht, Ruud Leliveld

Druk: Krips Repro Meppel

Ontwerp omslag: Buro Indruk 


\title{
INFANTS WITH ASTHMA
}

\section{TOWARDS A MULTIDISCIPLINARY EDUCATION PROTOCOL FOR PRIMARY CARE}

\begin{abstract}
PROEFSCHRIFT
ter verkrijging van de graad van doctor aan de Rijksuniversiteit Limburg te Maastricht, op gezag van de Rector Magnificus, Prof. Mr. M.J. Cohen, volgens het besluit van het College van Dekanen, in het openbaar te verdedigen op vrijdag 25 juni 1993 om 16.00 uur

door
\end{abstract}

\section{Ellsabeth Plerre Evellne Mesters}

geboren te Den Haag op 6 mei 1964

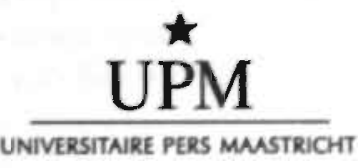




\section{Promotores:}

Prof. Dr. G.J. Kok

Prof. Dr. G.S. Parcel

Prof. Dr. H.F.J.M. Crebolder

\section{Co-promotor:}

Dr. R.M. Meertens

\section{Beoordelingscommissie:}

Prof. Dr. H. Huijer Abu-Saad (voorzitter)

Prof. Dr. Th,L. Creer (Ohio University, Athens)

Dr. A.A. Kaptein (Rijksuniversiteit Leiden)

Prof. Dr. J.A. Knottnerus

Prof. Dr. H. Philipsen 


\section{Acknowledgements}

"Nothing ever becomes a success unless exuberant spirits have helped to create it."

Friedrich Nietzsche

There are many people who have contributed to the different studies. First, I would like to gratefully thank the general practitioners, the community nurses, the asthma nurses, the doctors of child health care centers and, of course, the parents of the children with asthma for their participation in the various studies. Without their positive contributions my research would simply have been impossible.

I am greatiy indebted to my co-promotor Ree Meertens for her valuable advice on the design of the studies. She also supervised me while I did the several studies and helped me to write numerous annual reports, grant propesals, continuation grant proposals, abstracts, articles, and last but not least, the underlying thesis. Dear Ree, I have immensely appreciated your critical comments through the years.

I would like to thank my promotors, Gerjo Kok, Guy Parcel and Harry Crebolder, for their helpfulness in advising me on various research problems.

I am most grateful to the Netherlands Asthma Foundation for the financial support for the studies as described in this thesis.

To the reviewers of this thesis, the anonymous reviewers of the published articles and the anonymous reviewers of the Dutch Asthma Foundation, I would like to express my gratitude for al! the suggestions they made; they changed this thesis for the better.

Ger Houben, !ka Ploumen and Marcel Pieterse are credited for their practical assistance at several important moments.

I would also like to thank Marjo Franssen, Bram de Wit and Huib Franssen for their willingness to guide the group sessions during the pilot study.

Paul Hőppener and Martijn van Nunen, your spontaneous readiness to help me 
to collect medical consumption data from your network has made this part of my research very agreeable.

The following people have contributed in their own way on specific parts of the project (in alphabetic order): Buro Indruk, Jan van Dale, Gregor Franssen, Han Hendriks, Syb van der Meer, Elleke Knapen, Prof.dr. K. Knol, Neeltje Mosterd, Jean Muris, Thomas Plaut, the students that conducted the telephone interviews (especially Monique van Mierlo), Thomas Siwaak and Bob Wilkinson.

And finally, I would like to thank my colleagues, especially Margo, Aart, Maria, Marlein, Marja, and Manon, for making work such a pleasant thing to pass the time.

Maastricht, March 1993 


\section{Contents}

1 Overview $r 11$

$\begin{array}{lll}1.1 & \text { Introduction } & 11\end{array}$

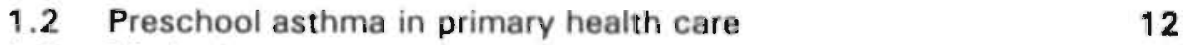

$\begin{array}{lll}1.3 & \text { Methods } & 14\end{array}$

1.3.1 Organization: eliciting and devising education tasks $\quad 14$

1.3.2 Content: tailoring education to the target population $\quad 17$

$\begin{array}{ll}\text { 1.3.3 Transfer: protocol design } & 18\end{array}$

$\begin{array}{ll}\text { 1.3.4 Pilot study on program effectiveness } & 19\end{array}$

1.3.5 Impiementation and evaluation in primary care 20

1.4 Summary of results $\quad 22$

1.4.1 Study on organization $\quad 22$

$\begin{array}{ll}1.4 .2 \text { Study on content } & 23\end{array}$

$\begin{array}{ll}\text { 1.4.3 Pilot study } & 25\end{array}$

1.4.4 Evaluation study $\quad 25$

1.5 General discussion 28

1.5.1 The protocol's development 28

1.5.2 The protocol's implementation 31

1.5.3 Implications for education protocol development $\quad 32$

1.5.4 Significance of education protocols 34.

$\begin{array}{ll}\text { 1.5.5 Final remarks } & 35\end{array}$

2 Multidisciplinary cooperation in primary care for asthmatic

children

2.1 Introduction 38

2.2 A systematic way to go about the question "who does
what"

2.3 Technique applied to asthma $\quad \mathbf{4 1}$

2.3.1 Asthma in young children $\quad 41$ 
$\begin{array}{ll}2.3 .2 \text { Method } & 41\end{array}$

$\begin{array}{ll}2.3 .3 \text { Sample } & 42\end{array}$

2.3.4 Data analysis $\quad 43$

2.4 Results $\quad 44$

2.4.1 Demographic data 44

2.4.2 Task conceptions and knowledge $\quad 45$

2.5 Conclusion 48

3 Pediatric asthma, a qualitative and quantitative approach to needs assessment $\quad 51$

Abstract 51

3.1 Introduction 52

3.2 Childhood asthma $\quad 52$

3.3 Focus group interview

3.4 Methods 53

3.4.1 The questionnaire 53

3.4.1.1 Instrument 53

3.4.1.2 Data collection $\quad 54$

3.4.1.3 Data analysis $\quad 54$

3.4.2 The focus group interview $\quad 54$

3.4.2.1 Instrument $\quad 54$

3.4.2.2 Data collection $\quad 55$

3.4.2.3 Data analysis $\quad 55$

3.5 Results $\quad 56$

3.5.1 Subjects $\quad 56$

3.5.2 Prevention $\quad 56$

$\begin{array}{ll}3.5 .3 \text { Medication } & 57\end{array}$

3.5.4 General aspects of asthma 60

3.5.5 Perceived experiences and satisfaction with the

3.6 Discussion 63

4 Development of a health education program for parents of

$\begin{array}{ll}\text { preschool children with asthma } & 67\end{array}$

$\begin{array}{ll}\text { Abstract } & 67\end{array}$

4.1 Introduction 68

4.2 Needs assessment studies $\quad 70$

4.3 Method 72

4.3.1 Program development $\quad 72$

$\begin{array}{ll}4.3 .2 \text { Pilot study } & 73\end{array}$

4.3.2.1 Population $\quad 74$

4.3.2.2 Instrument development $\quad 75$

4.3.2.3 Data collection $\quad 76$

4.3.2.4 Data analysis $\quad 76$

$\begin{array}{lll}4.4 & \text { Results } & 76\end{array}$

4.5 Discussion $\quad 77$

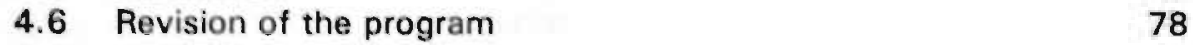

viii 
5 The effectiveness of a multidisciplinary education protocol for

children with asthma (0-4 years) in primary health care

Abstract

81

5.1 Introduction

5.2 Methods

5.2.1 Recruitment and randomization 84

5.2.2 Data collection procedures 86

5.2.3 Measurement instruments 86

$\begin{array}{ll}\text { 5.2.4 Analysis procedures } & 88\end{array}$

$\begin{array}{lll}5.3 & \text { Results } & 88\end{array}$

5.3.1 Sample characteristics 88

$\begin{array}{ll}\text { 5.3.2 Process evaluation } & 89\end{array}$

5.3.2.1 Transfer of education $\quad 89$

5.3.2.2 Cooperation between disciplines $\quad 91$

5.3.3 Impact and outcome evaluation 92

5.3.3.1 Impact evaluation: Educational and behavioral variables 94

5.3.3.2 Sociodemographic variables $\quad 97$

5.3.3.3 Outcome evaluation: Health status indicators 98

$\begin{array}{ll}5.4 \text { Discussion } & 100\end{array}$

$\begin{array}{ll}5.5 & \text { Implications for practice } \\ \end{array}$

$\begin{array}{ll}\text { References } & 105\end{array}$

$\begin{array}{ll}\text { Summary } & 115\end{array}$

$\begin{array}{ll}\text { Samenvatting } & 119\end{array}$

$\begin{array}{lr}\text { Appendix } 1 & 123\end{array}$

$\begin{array}{lr}\text { Appendix } 2 & 131\end{array}$

$\begin{array}{lr}\text { Appendix } 3 & 133\end{array}$

Appendix 4

$\begin{array}{lr}\text { Appendix } 5 & 141\end{array}$

$\begin{array}{lr}\text { Curriculum Vitae } & 143\end{array}$ 


\section{Overview}

\subsection{Introduction}

This thesis describes the development, implementation and evaluation of an education protocol for parents of children (0-4 years) with asthma. The education protocol is intended to enable health care providers to provide systematic and coordinated education to asthma patients and their parents. Today the relevance of adequate education for patients with asthma will hardly be under discussion. For children with asthma, research has shown that adequate education has a positive impact on their self-management of asthma; favorable clinical effects have been revealed as well (Wigal, Creer, Kotses \&! Lewis, 1990). It is accepted that patient education should be integrated in the overall care for patients, but how this should be achieved remains rather unclear. Education protocols have been suggested as a promising way to achieve a systematic and coordinated approach to patient education in routine care. An education protocol has several functions IDekkers, 1981; Kanters \& Jonkers, 1988). The present education protocol arranges the organization of the patient education, the content of the patient information, as well as the transfer of information from care providers to patients. The organizational function of the protocol is to give directions about who will provide what education. It might also indicate how activities can be coordinated so that cooperation between disciplines and continuity of education can be achieved. Directions about the content of the patient information can be restricted to the synopsis of the subject matter of the information. This means that the protocol suggests what information has to be provided to people having a certain disease. Finally, the third function of the protocol addresses ways of transferring patient education to the target population, such as techniques to enhance communication.

Even though we know of what elements an education protocol should consist, there is limited experience with the development of such protocols. This thesis is 
one of the first examples that shows the systematic steps that are to be undertaken to develop an education protocol. Within this scope children with asthma $(0-4$ years of age) in primary health care serve as an example.

This chapter will only discuss the most essential points of the steps undertaken and the main results obtained, followed by the most important methodological and practical implications that result from the studies conducted. Those readers interested in more detailed information are referred to the respective chapters. For an education protocol to be effective, its development, implementation and evaluation require a planned approach (PRECEDE ${ }^{1}$ model: Green, Kreuter, Deeds \& Partridge, 1980; Kok, 1988; PRECEDE-PROCEED ${ }^{1}$ model: Green \& Kreuter, 1991). The planning process was started with a diagnosis of the health problem asthma among young children in primary care. Thus, the beginning of this overview provides background information on childhood asthma and the motives for the focus on preschool children and on primary health care. The remainder of this overview describes the studies conciucted to develop the education protocol. After the identification of health care providers, relevant for the delivery of asthma information to parents of young children, the first study was conducted. This study, which is described in more detail in Chapter 2, focussed on the division of education tasks among the health care providers. who provide care for young children with asthma. Then, the next study concentrated on eliciting opinions, interests „misconceptions and needs of parents of children with asthma (Chapter 3). On the basis of the first two studies the protocol could be developed. After a description of the initial pretest of the protocol (Chapter 4), its implementation into primary health care, and the assessment of the effects of the protocol at posttest and one year follow-up, are reported (Chapter 5). Process, impact and health outcomes are evaluated. Process evaluation questions whether the implementation has been successful. Impact evaluation concentrates on the question whether the behavioral variables have changed. Health outcome evaluation concentrates on changes in the variables relevant for the prevalence of asthma among preschool children.

\subsection{Preschool asthma in primary health care}

Asthma is defined as recurrent episodes of wheezing or dyspnea characterized by a significant increase in resistance to airflow. Spontaneously or following treatment, episodes of complete or almost complete freedom of symptoms occur accompanied by a substantial decrease in resistance in airflow (Busse \& Reed, 1988). Asthma is the most prevalent chronic disease among children. Asthma can not be cured. This makes management over time by health care providers in collaboration with patients (and parents) necessary. For asthma, it is expected that early detection and

\footnotetext{
'PRECEDE stands for 'predisposing, reinforcing, and enabling causes in the educational diagnosis and evaluation'; PROCEED stands for 'policy, regulatory, and organizational constructs in educational and evironmental development'.
} 
adequate treatment might prevent or reduce future symptoms (Kerrebijn, Hoogeveen-Schroot \& Van der Wal, 1976). Health care providers can make an important contribution to the detection of this disease at an early stage and to an adequate treatment. On the other hand, patients can make a difference by cooperating with their care providers and by adherence to the advised regimens. Yet, adequate patient education from health care. providers to patients is vital in the patient's management of a disease. How, for instance, can patients use prescribed medicines correctly if the appropriate procedures to follow are not explained to them or not understood well enough.

For patients, self-management means having, or being able to obtain, the skills and resources necessary to best accommodate the chronic disease and its consequences (Holroyd \& Creer, 1986). In the past decade a variety of education programs have been developed to teach self-management skills to elementary school-aged children (and their parents) (Rachelefsky, 1987). However, programs addressed to the very young asthma patient (0-4 years old) were not found in literature, whereas it is known that most children experience asthma symptoms before they reach the age of four (Gerritsen, 1989a). Colland (1990) concluded in a Dutch study that parents expressed that their need for asthma information had been especially high when their child was between 0-6 years old and/or in the period that they were told that their child's symptoms could be ascribed to asthma. Furthermore, trend analysis of age specific hospitalization figures in the Netherlands has shown a decrease in hospital admissions due to asthma in the period 1980 1988, except for 0-4 year olds: (Wever \& Wever-Hess, 1991). In this age group a considerable increase in hospitalizations was found, which could, according to these authors, not only be attributed to changes in labels for asthma, increased incidence of asthma, increased prevalence of asthma or increased asthma severity and/or changes in treatment. Starting from the assumption that education and selfmanagement is needed when asthma is diagnosed by a physician, these findings seem to indicate a strong need for a self-management program for this age group. Because our target group, 0-4 year olds, is too young to assume responsibility for the self-management of their asthma, the construct self-management will refer in this thesis to parentsi management of their child's disease. Similarly, patient education refers to the education of the parents.

The best setting for which to develop a protocol is the one in which most children can be reached. Asthma self-management programs have been developed for several settings: hospitals, outpatient clinics, schools, camps and primary health care (Rachelefsky, 1987; Klingelhofer \& Gershwin, 1988). Self-administrative programs also exist (Weiss, 1981). In clinical settings one might expect to find more severely affected asthmatic children, but this represents only a small proportion of the target population. Other settings, such as schools and camps, provide the opportunity to reach more children and also less severely affected children. However, these programs are only conducted occasionally and they often do not involve systematically the patients' health care providers. The latter point is considered important for self-management programs to have beneficial effects on 
morbidity, health behavior outcomes and quality of life in asthmatic patients (Schlösser, 1992). Health care services provided on an ambulatory basis offer several opportunities for implementing education programs. In the Netherlands, as in many other countries, most asthma patients $(80 \%)$ can be reached for providing systematic patient education in primary care physician's offices (Voorn, 1983; Gregg, 1985; Schipper, 1988). Asthmatic patients consult their general practitioner (GP) frequently (Centraal Bureau voor Statistiek, 1986). In the Netherlands both mild, moderate and severely affected children are looked after by the GPs, although as might be expected, more severely affected children are more likely to be referred to the pediatrician or pulmonologist. Furthermore, in Dutch primary care, local health departments (child health centers) provide community-based services. These services include regular check-ups to an average $75 \%$ of the $0-4$ year old young children (Geradts, 1987). Finally, primary health care is the setting in which most children with asthma can be reached.

\subsection{Methods}

\subsubsection{Organization: eliciting and devising education tasks}

We already mentioned that the first study focussed on the organization of the patient information. The set-up of this study, which is described in further detail in Chapter 2, was as follows. First, a literature study was carried out to reveal an outline of asthma information, based on existing programs, that might be relevant for parents of young children with asthma. Then, the quality of these programs was judged on their effectiveness. Wigal et al. (1990) concluded after reviewing the development and evaluation of 19 self-management programs for childhood asthma that despite some shortcomings, overall, the programs produced positive changes in the lives of asthmatic children and their parents. Thus, with some confidence in its adequacy, the content of the protocol could be based on the already available programs. The next step was aimed at selecting the health care providers who are involved in the care of preschool children with asthma. Besides GPs, community nurses, asthma nurses and doctors of child health centers were considered important because they were most likely to see these children.

GPs in the Netherlands may be seen as gate-keepers of the health system; as a rule patients consult GPS first for their symptoms, and are referred to the specialist as needed. Community nurses, asthma nurses and the doctors of local child health centers are employed by arganizations for home care. These are regional organizations that provide home care for patients. One of the functions of these organizations is to provide preventive care for the 0 -4 year-old children through loca! child health centers. The community nurses and the doctors in these centers are the professionals who provide direct care to people from their local community. The asthma nurse is a staff member in the organizations for home care who is responsible for helping and advising health care providers and the management of 
the organization on asthma related problems.

At this point of the study we summarized the basic information parents of children with asthma need to. Furthermore, the most important disciplines were identified. Next, we needed to determine which discipline was going to provide what part of the information. A search of literature did not reveal a clear and detailed description of education tasks on asthma for the disciplines involved. Also, no systematic way to divide tasks among disciplines was found. Therefore, the following method was developed. From the total picture of relevant asthma information we developed a questionnaire on task conceptions about asthma education. For easy reference we consolidated the tasks we distinguished in several umbrella categories: (1) "noticing asthma symptoms", (2) "other asthma related problems", (3) "adaptation of the home", (4) "giving information on medication", (5) "providing basic asthma knowledge", (6) "general advice on asthma", (7) "referral of patients to other disciplines", (8) "coordination of care" and (9) "taking a refresher course". The first six categories dealt with the provision of patient education. The last three categories contained tasks that were beneficial to the provision of education. Our aim was to develop an education protocol which would be widely applicable. The written survey on task conceptions was therefore conducted nationwide on representative samples of 100 care providers per discipline, except for the asthma nurses. For this category all 58 nurses who worked in the cooperating organizations in the Netherlands were approached.

The information gathered in this survey was used as a basis for the division of education tasks across the disciplines involved. If care providers are expected to use the protocol in a cooperative way, it is evident that they should (at least to some extent) agree with the task assigned to them and to others. Furthermore, to make the cooperation between disciplines a success, care providers should have the essential knowledge to adequately perform assigned education tasks. Care providers could indicate in the questionnaire which tasks they considered relevant for their discipline. Furthermore, they could indicate what tasks they would assign to the remaining disciplines. Finally, they had to complete a knowledge test. The knowledge measure consisted of a series of subsets, which each referred to the knowledge needed for a specific task. In this way it would be possible to find out if a certain discipline had enough knowledge to perform a certain education task. The knowledge questionnaire was reviewed by an expert in childhood lung diseases for medical relevance and accuracy before administration to the disciplines. Tasks were considered relevant for a discipline if they were seen relevant by about two thirds of the respondents of one discipline. Next, agreement with opinions of other disciplines was checked. Again, at least two thirds of the other disciplines combined should assign this task to the discipline in question. Finally, available knowledge was studied to estimate the likelihood that a discipline could appropriately perform an assigned task. For this purpose at least $60 \%$ of relevant knowledge questions needed to be answered correctly. Based on these three criteria eight outcomecombinations were distinguished, each combination indicating different implication for the protocol. These combinations are depicted in Table 1. As can be derived 
from this table, assignment was merely based on the claim of tasks. Knowledge was not considered a major barrier for assignment, because deficits could usually be solved by training.

Table 1

Possible outcome-combinations based on three criteria

\begin{tabular}{|c|c|c|c|c|}
\hline Combination & $\begin{array}{l}\text { Task claimed } \\
\text { by one discipli- } \\
\text { ne in question }\end{array}$ & $\begin{array}{l}\text { Agreement of } \\
\text { other discipline }\end{array}$ & $\begin{array}{l}\text { Knowledge of } \\
\text { the discipline } \\
\text { in question }\end{array}$ & $\begin{array}{l}\text { Implications for the } \\
\text { protocol }\end{array}$ \\
\hline A & yes & yes & yes & Consider assignment \\
\hline B & yes & yes & no & $\begin{array}{l}\text { Consider assignment and } \\
\text { augment knowledge } \\
\text { through training by giving } \\
\text { information in the protocol }\end{array}$ \\
\hline C & ves & no & ves & $\begin{array}{l}\text { Consider assignment when } \\
\text { cooperation will not cause } \\
\text { problems }\end{array}$ \\
\hline D. & yes & no & no & $\begin{array}{l}\text { Consider assignment when } \\
\text { cooperation will not cause } \\
\text { problems, and knowledge } \\
\text { can be augmented by } \\
\text { giving information in the } \\
\text { protocol or through training }\end{array}$ \\
\hline$E$ & no & no & no & $\begin{array}{l}\text { Assignment not recom- } \\
\text { mended }\end{array}$ \\
\hline$F$ & no & no & yes & $\begin{array}{l}\text { Assignment not recom- } \\
\text { mended }\end{array}$ \\
\hline G & no & yes & no & $\begin{array}{l}\text { Assignment only recom- } \\
\text { mended after consulta- } \\
\text { tion with, and acceptance } \\
\text { by the disciplines in ques- } \\
\text { tion }\end{array}$ \\
\hline H & no & yes & yes & $\begin{array}{l}\text { Assignment only recom- } \\
\text { mended after consulta- } \\
\text { tion with, and acceptance } \\
\text { by the disciplines in ques- } \\
\text { tion }\end{array}$ \\
\hline
\end{tabular}




\subsubsection{Content: tailoring education to the target population}

Patient education is usually regarded as an important element of programs to manage a chronic disease, such as asthma. In general, information is provided to asthma patients about the disease's symptoms, pathophysiology, course, outcome, and treatment. Patients who have more knowledge about their disease are expected to be in a better position to participate in the management of the disease (Mazzuca, 1982). Literature suggests that knowledge about asthma among parents of asthma patients is not optimal (Spykerboer, Donnelly \& Thong, 1986; Donnelly, Donnelly $\&$ Thong, 1987). Thus, providing knowledge about asthma to parents who are confronted with this disease seems logical. However, attitude development and skill acquisition is needed in addition to gain in knowledge. As Donnelly et al. (1987) stated, it is difficult to say whether or not negative attitudes about asthma would lead to poor management strategies on the part of the parents. Nevertheless, positive health beliefs and attitudes have been shown to correlate positively with medication compliance (Radius et al., 1978; Smith, Seale, Ley, Shaw \& Bracs, 1986). Of course, an optimal medication regimen is a cornerstone in the treatment of asthma. Research has shown that parents' attitude towards asthma and their attitude towards treatment should be improved (Donnelly et al., 1987). An important treatment goal of our protocol is therefore to enhance those parents' beliefs about asthma and its treatment that promote effective self-management. Selfmanagement refers to the individual assuming preventive or therapeutic health care activities, often in collaboration with health care professionals (Holroyd \& Creer, 1986). One can distinguish between the actual performance of the self-management behaviors to prevent and manage attacks, the self-management skills, and the parents' perception of their capability to perform the behaviors required. With respect to skill acquisition, techniques have been recommended to providers of the education to increase the impact of education programs. These techniques will be described in more detail in the next paragraph ( $\$ 1.3 .3$ ). The moment when someone tries to perform the actions learned within a patient education program, it is important that the person believes that he can successfully perform the activities required. It is suggested that such beliefs may predict which persons may be more successful in managing a physical disorder, such as asthma (Clark \& Zimmerman, 1990; Creer \& Kotses, 1990). Therefore it may not come as a surprise that the underlying asthma protocol also focuses on increasing the perceived self-efficacy of parents with respect to the management of asthma. In conclusion, a gain in psychological factors such as knowledge, attitude and self-efficacy can only be called successful if parents also change their self-management behaviors in the desirable way. Ultimately, education should lead to maintenance of the desired behavior.

As mentioned before, the content of the education protocol was based on established asthma programs (mostly for children 8-12 years in the USA). The content. of these programs needed to be tailored to a younger target group living in the Netherland's. The aim of the third chapter is to describe how components of existing 
asthma education programs were adapted to the needs, ideas and (mis-) concep-tions of parents of children aged 0-4 years in the Netherlands. Such information on needs, ideas and misconceptions may be gathered by a written questionnaire. However, a common handicap of gathering data with such an instrument is that important issues such as misconceptions cannot always be anticipated and consequently are not included in the questionnaire. Therefore, techniques that allow a more open response mode, such as focus group interviews, might serve as a complementary method in assessing educational needs of the target population. The focus group method, which is a qualitative research method, enables the researcher to learn from the participants in the study; the respondents are regarded as experts (Krueger, 1988). Thus both methods, a written questionnaire and six focus groups interviews, were used to conduct the needs assessment among 42 parents representing 26 children with asthma.

\subsubsection{Transfer: protocol design}

A partnership between the 'patient' and the health care provider is regarded essential for appropriate self-management of asthma (Holman \& Lorig, 1992). Models of patient-provider relationships might be useful in understanding and subsequently improving the communication between parents and their care providers. Joos \& Hickam (1990) have summarized several empirically effective behaviors to enhance communication. These behaviors were derived from social-psychologica! perspec-tives on patients-provider interaction. Several of these behaviors were incorporated in the design of the protocal, which will be described next.

The transference of information from care providers to parents in the protocol was organized in a way that was easy for both care providers and patients; the education protocol, for example, consisted of written materials ${ }^{2}$ for both groups. The materials:

1) Manuals. A sppecific manụal was developed foi every disccipline involved. In these manuals the educational and organizational tasks of each discipline were described. For instance, whenever a GP should involve a nurse in the asthma care, this was indicated in the manual. To help health care providers notice these tasks, a specially designed symbol was printed in the margins of the manuais. A referra! was indicated by a symbol of an index finger pointing at the referral task. The manuals also focussed on critical components of providing patient education, such as the necessity of problem clarification, the importance of checking patient understanding and the necessity of targeting priorities per contact. Furthermore, it was stressed that formulating concrete aims was essential. The importance of involving parents in developing the management plan by eliciting their perspectives so that desired

\footnotetext{
2 The education materials, which are written in Dutch only, are available free of charge through the Netherlands Asthma Foundation, Department of Patient Education, PO Box 5, 3830 AA Leusden. the Netherlands.
} 
goals would be mutually acceptable, was emphasized. Also, it was suggested in the manuals to evaluate with parents the actions they have performed. In addition, the physician's manuals contained an overview of medication prescribed for young children with asthma and a diagnostic protocol.

2) Checklists. In the manual the patient information content was summarized by means of keywords. These keywords were printed on separate cards, which functioned as checklists. These checklists were intended for the health care provider who had some experience with the protocol. The checklists were used during contact with the parents.

3) Parent booklet. The parent booklet contained basic asthma information. Because it was expected that a considerable proportion of patients would be unable to recall (all of) what they were told, the protocol paid special attention to the use of written information for parents. The parent booklet consisted of 16 education modules and could be handed out in a flexible way. The GP could select the amount and kind of information by extracting relevant pages (modules) from the parent booklet at the time of contact: with a parent, and subsequently provide the extracted modules to the parents. Parents could read the information at home. In a next contact parents could discuss questions they still had with the relevant care provider. The care giver in question might give parents tailored information (through discussion of the modules) to complement the standard written material. Care providers had to check parents" comprehension of the asthma information to make sure that parents really understood the information they read. An overview of what discipline was going to discuss which modules was indicated in the manuals. After the consultation the modules were to serve as a reminder for parents.

Instructions were written down as specifically as possible. For instance, the booklet would state 'clean the floor once or twice a week', instead of 'clean the floor regularly'. To stimulate parents to express their ideas, questions and concerns about asthma, space was reserved in the written materials in which parents could write the questions they wanted to discuss in the next contact with the care provider.

Every topic in the parent booklet was rephrased at the end in terms of action to ensure that parents would know what actions they could take and to encourage the feeling that 'they could do it'. Establishment of the conviction among parents that prevention, early detection and treatment of asthma symptoms were necessary, was a major treatment goal. Finally, health care providers could use the parent booklet for themselves to get an impression of what information they could provide to parents.

\subsubsection{Pilot study on program effectiveness}

Chapter 4 describes the design and results of a pilot study of the education protocol in a group setting. Orlandi, Landers, Weston \& Haley (1990) identified barriers which have the potential, if left unaddressed, to undermine the objectives of either 
the protocol-development or the protocol diffusion process. From a broad perspective the protocol could fail at four points. First, the content may be unsuitable to bring about its intended effects. For instance, the parents do not understand the textual or graphical information, or the information is not convincing enough. Illustrations and information that do not fit to target population's ideas will diminish the effects of the protocol. Second, a program that has the potential to achieve its intended effects, can fail to do so because its developers are ineffective in communicating its applicability to the users. Third, despite the fact that the care providers are well informed about the applicability of the protocol it may be implemented inadequately by the health care providers or not used at all. This means for instance that they do not refer patients to other disciplines when necessary, they omit the discussion of the information with parents or they provide the information inadequately. The last critical point is program maintenance over time; initial users of the program may stop using the program after some time. It was decided to conduct a pilot study to ensure that the implementation would not result in a failure related to the content of the protocol. How the remaining potential failures were addressed is described in \$1.3.5.

The pilot study was conducted among a limited part of the target population. Parents of 31 children with asthma participated in the study. The content of the protocol was checked in three ways for the existence of failures or shortcomings. First, representatives of each discipline and an expert in pulmonary diseases were asked to review the protocol. Second, the content of the protocol was provided to parents whose children were diagnosed as having asthma. Educators, especially recruited for this job, provided information during three (weekly) group sessions. In this situation the amount and the way the information was provided could be controlled. The program was evaluated with a one group post- and pretest design. Data were collected among parents by means of a written questionnaire. This questionnaire is enclosed in Appendix 3. It measured outcomes with respect to knowledge, attitude, self-efficacy, self-management behaviors and state-anxiety. Third, the trained educators were interviewed to reveal their experiences with the protocol.

\subsubsection{Implementation and evaluation in primary care}

Chapter 5 focusses on the implementation and the process, impact and outcome evaluation of the protocol at 6 months posttest and one year follow-up.

In the implementation phase the protocol was used by the health care providers in primary health care. When preparing the dissemination and implementation of the protocol, consideration was given to the organizational structure in which the disciplines involved were employed. A distinction was made between disciplines which were self-empioyed and worked in an established practice and disciplines that were employed by a (hierarchical) organization. GPs fell within the first mentioned category, the others fell in the second category. First, the GPs were recruited. Since 
GPs can decide for themselves in what studies they want to be involved, they were recruited in a direct way (regiona! newsletter, personal mail, telephone calls and personal visits). GPs who were interested in participation enrolled parents for the study by providing them with an entry form. This form could be returned by the parent to the research team. On the entry form parents were asked to indicate the child health center which they visited for regular check-ups. Because organizations for home care in which these disciplines are employed is hierarchically structured, a coordinator was sought within each organization. The asthma nurse was most suited to the criteria for program coordinator (Steckler \& Goodman, 1989). The research team approached the asthma nurses to discuss ways of recruiting community nurses and doctors within the cross association. Next, the asthma nurses contacted the executive officials of both disciplines, who in turn contacted the relevant community nurses and doctors at the child health care centers. In all, 108 patients and their GPs ( $N=67$ ) participated in the study. For the treatment group 40 community nurses, 12 asthma nurses and 26 doctors of child health centers were recruited. The. GPs (and with them all related patients and remaining care providers) were randomly assigned to either treatment $(N=35)$ or waiting list control group $(N=32)$.

During the enrollment phase both parents and GPs were informed on paper about the fact that they would either be assigned to a group that would get the intervention first or to a group that would receive the intervention after the data collection periods were over. Thus, the parents in the control group knew that they had to fill out the pretest and posttest questionnaire before they would receive the intervention.

The following introduction strategies, based on diffusion theory (Rogers, 1983), were used during the introduction of the program in the experimental group. Rogers identified several features of an innovation that will influence the speed and the degree in which it will reach its target population: compatibility, relative advantage, complexity, trialability and observability. Respectively, the higher the compatibility of the product with values, needs or norms of the target population, the higher the perceived relative advantage of the protocol and also the more easily the protocol can be used, the more easily it will be implemented. New ideas that can be tried on a limited basis or scale are more likely to be adopted. And finally, the more visible the positive results are to others, the more communication about the product is stimulated, which will promote greater adoption and diffusion of an innovation. Care providers received the education materials and written instructions two weeks before the official introduction of the protocol, which was carried out by a member of the research team. The aim of the personal visit was to ensure that the care providers had adequate information on how to use the protocol and the protocol's potentials. The message, addressed to the care providers by word of mouth and in writing, referred to the participation of their occupational group in the development of the protocol. The advantages, like favorable behavioral effects, more satisfied patients and automatic administration of the information provided, were stressed. The uncomplicated use of the protocol was demonstrated; care providers 
could already start using the protocol with only one patient and the care providers were told to look for positive signs such as better self-management by parents. Finally, it was emphasized that the assignment of tasks indicated the main responsible person, which did not mean that a care provider who wanted to discuss non-assigned information with parents, was not allowed to do so.

After our grant for collecting long term data (behavioral data, process data and medical consumption data) had been honoured by the Netherlands Asthma Foundation we approached the experimental group (care providers and patients) by letter and telephone to ask if they were willing to participate once more in our study. Because the control group had had the intervention as well after the data collection periods were over, they could no longer function as control group for the medical consumption data. Therefore, the control group was only asked to fill out a short questionnaire for the purpose of collecting process data. These questions were the same as the ones the experimental group filled out at the posttest. With respect to the medical consumption data a new control group of GPs was sought and found in a part of a group of GPs who already were linked to a regional registration network of family practices (Metsemakers, Höppener, Knottnerus, Kocken \& Limonard, 1992). After permission was obtained from the coordinator of this network, GPs were informed in writing about our intention to collect medical consumption data on some of their asthma patient. The GPs in question were contacted by telephone to see if they were willing to participate.

\subsection{Summary of results}

\subsubsection{Study on organization}

In the development of the education protocol on preschool asthma, educational tasks had to be divided among four disciplines. A technique for doing this was based on three criteria: whether the discipline in question claimed a certain task, whether the other disciplines agreed with this claim, and whether the discipline in question had enough knowledge to perform the claimed task. The results of the survey on the organization of the patient education are described in this thesis as follows. First, an account is given of the division of tasks by means of a more detailed description of the method of assigning tasks among several disciplines in Chapter 2. The data on community nurses will serve as an illustration of the method in question. It needs to be noted that although the community nurses were used to illustrate the technique applied, this does not imply that the community nurse is more important in asthma care than the other disciplines involved. The data of the other three disciplines are included in Appendix 1. Furthermore, an overview of the final division of the tasks among disciplines is provided in Appendix 2. Response rates for the questionnaire used varied among disciplines from $51 \%$ to $82 \%$. The general conclusion of the survey was that with the method employed most tasks could be divided among the disciplines involved. With some tasks; 
besides the criteria mentioned earlier, task overload of a discipline and match of tasks were considered as well. As deficits in care providers' knowledge about asthma were revealed, it was decided to address these knowledge deficits in the protocol by providing information about asthma for care providers. The key person in the asthma care for $0-4$ year olds proved to be the GP. Tasks concerning involvement of other disciplines in the care, diagnosis, medication and basic information about causes of asthma were assigned to this discipline. The results of the study furthermore showed that community nurses should have an important role in adapting the home and practicing with patients in the use of their asthma medication. However, as the relevant knowledge of the community nurses seemed to be lacking, community nurses are advised to ask the asthma nurse to join them in their first home visit. Doctors in local child health centers had the least tasks assigned to them, their most important function was to detect asthma symptoms and to refer patients to other disciplines in case of suspicion of asthma or asthma related problems.

\subsubsection{Study on content}

The intention of the next study was to carry out an educational needs assessment for parents of 0-4 year-old children with asthma because no specific information on their level of knowledge, needs concerning training in the self-management of asthma and (mis-) conceptions about asthma were available. The subsequent focus group interviews revealed interesting misconceptions about asthma and the questionnaire provided additional quantitative information on parental knowledge and misconceptions. Some interesting differences between data obtained by questionnaire and the focus group interview were found. For instance, in the questionnaire fewer parents reported a negative evaluation of the quantity and quality of the information received in routine primary care than during the interviews. Talking about experiences appeared to stir up a commotion among parents about negative incidents that were almost forgotten, while giving an answer in writing created more distance to the matters in question. Such differences indicated the typical value of both methods in assessing educational needs of the target population.

To guarantee some objectivity regarding what should be labelled as misconceptions and other interpretations from the qualitative data, the interview data were transcribed and reviewed separately by two researchers. Furthermore, the misconceptions were reviewed by a pediatrician specialized in asthma. The present findings indicated that parents' knowledge seemed in need of improvement. Furthermore, parents appeared to have an inadequate understanding about how to apply the information they had received. Additionally, they felt that they lacked objective indicators, for instance they wanted to know how to monitor the severity of an asthma attack. It was intriguing that a lot of misconceptions and negative beliefs dealt with medicines for asthma. Moreover, it was revealed that parents felt that they were left to their own devices in learning about asthma self-management. An 
interactive relationship between care provider and parent was viewed as lacking. An example of parents' thoughts is that some parents believed that their child was getting addicted or used. to the medication prescribed. Further questioning revealed that the main reason why they thought so was that the GP regularly increased the dosage of the medication. However, what parents did not realize was that an increase mostly meant that the doctor adjusted the dosage to the increased weight of the child because it had grown. More detailed results are reported in Chapter 3.

Social learning theory (Bandura, 1986) can be used to name the variables that are likely to influence health behavior change and health education programs can be planned with these variables in mind. In short, social learning theory considers learning as a reciprocal interaction between the individual's environment, cognitive processes and behavior. The likelihood that people adopt a valued health behavior (such as asthma self-management behaviors) may rely on three cognitions: the expectancy that a life situation is threatening (asthma attacks), the expectancy that behavioral change will reduce the threat, and the expectancy that one is sufficiently skilled to adopt a beneficial behavior (such as giving medication) or quit a harmful behavior (such as smoking). Although social learning theory consists of several key constructs (Perry, Baranowski \& Parcel, 1990), the encouragement of perceived competence to perform a specific behavior (self-efficacy) is of particular interest. Several longitudinal studies have shown that self-efficacy is an important predictor of future behavior change (De Vries, 1989; Mudde, Kok \& Strecher, 1989). Furthermore, the many constructs of social learning theory provide ample clues for intervention to influence behavior.

Several social learning theory-constructs were applied to represent the focus group findings. The fact that parents lacked the knowledge and skills to perform asthma self-management behaviors, which is called behavioral capability in social learning theory, demonstrated a need for systematic information from health care providers. However, adequate behavioral capability does not automatically guarantee adequate performance. According to social learning theory, outcome and efficacy expectations might influence the actual behavior of parents as well. Outcome expectations are a persons' beliefs concerning the effects of his or her action. Such beliefs, for instance about effectiveness of medicines, might interfere with an adequate management of asthma by parents. Therefore, such beliefs were addressed in the protocol in such a way that misconceptions were solved. Selfefficacy expectations are a person's beliefs about his or her capability to perform specific behaviors, for instance controlling asthma attacks. Such beliefs are considered to be related to whether or not a person will attempt a task and also to whether or not the performance will be attained. People with high self-efficacy are considered to be at an advantage in self-management in chronic diseases (Holman \& Lorig, 1992). To enhance a parent's confidence in performing management behaviors, the behaviors were presented in the written material in a step-by-step format. Guidelines on what to know and what to do were clearly distinguished in the information. Furthermore, in order to help the parent to monitor the severity of an asthma episode, indicated in social learning theory as the construct self-control, 
a scheme was included in the protocol that helped parents to check for signs of asthma trouble (for example number of respirations per minute) whether an asthma attack was mild, moderate or severe, as well as the progress of an attack.

For asthma patients the construct of self-efficacy might be interesting for additional reasons. Self-efficacy makes a difference in how people feel, think and act. People who have a low self-efficacy are prone to (state-)anxiety when confronted with critical achievement demands such as controlling an attack. Of course asthma attacks are not always completely under personal control, but it might be expected that a sense of control diminishes situation-specific appraisals of anxiety. Studies on the role of anxiety in asthma indicate that people with a too high and too low level of state-anxiety showed a higher medical consumption pattern than people with a moderate level of state-anxiety (Kaptein, 1982). The findings of this needs assessment study were used to refine the content of the protocol to address the needs and misconceptions of parents of young children with asthma. For the later implementation of the protocol this means that the possibility that care providers cease using the protocol because the information (and the design) is not effective and/or does not appeal or apply to the parents, is reduced as much as possible.

\subsubsection{Pilot study}

A first check: on the materials developed was carried out by asking an expert in pulmonary disease among children as well as the representatives of each discipline involved to carefully judge the materials (parent booklet and materials for the disciplines) on practicability and feasibility. This was done before the materials were piloted in a group setting. No important barriers or failures were revealed. After the intervention the experiences of the trained educators with the protocol were elicited. As the educators were positive about the protocol, only some minor adaptions were made as a result of the educators' suggestions. The parents involved in the pilot study showed an encouraging improvement on the variables measured (knowledge, attitude, self-efficacy, self-management behaviors and state-anxiety), except for state-anxiety. Furthermore, the pilot survey was used to identify needed changes in the text or illustrations of the asthma education protocol. Comments of parents lead to some minor adaptations of the content of the parent booklet. The positive results of the pilot study provided sufficient legitimation for the implementation of the protocol in primary health care. In short, the results of the pilot study looked promising and are described more detailed in the fourth chapter.

\subsubsection{Evaluation study}

The next step is a description of the protocol's effects in primary health care. Process evaluation in the experimental group concentrated on revealing how many 
patients were reached, to what extent, how the information was provided and how referral between disciplines took place. Nearly all GPs also provided the parent booklet to the parents enrolled in the study. GPs handed out a satisfactory amount of information; the least distributed module (the medication chart) was still received by $54 \%$ of the parents. The data collected indicate that GPs largely complied with the protocol's directions concerning the transfer of the information. This means that they distributed the modules over several contacts. Nonetheless, a few physicians handed out the entire booklet at one time. This did not conform to the guidelines in the protocol. Of the GPs who gave (parts of) the parent booklet to the parents, the majority did discuss the information with the parents. This discussion nearly always occurred and was also scheduled for a contact that followed the consults in which the module(s) were distributed. Findings revealed that the majority needed about 4 to 5 consultations to hand out and discuss all relevant information.

The use of the manuals and checklists was investigated by asking health care providers whether they had used the manuals. Almost all health care providers went over the manuals. The workers within the cross organization were not: confronted very often with study patients. Therefore they did not have to use their manual and the checklists. Almost every GP used his manual and somewhat more than half the GPs used the checklists. Care providers who had used the educational materials stated that the communication with patients was improved by the use of the protocol. Overall, the health care providers rated the content and form aspects of the materials as very positive. This finding also applies to those providers who had not been able to use the materials. Another indication for health care providers' high satisfaction with the protocol was their positive intention to start using or to keep on using the protocol.

Long term evaluation on the degree and way of use of the protocol by care providers and parents in the experimental group revealed that the protocol was still in active use to a satisfying degree by parents (92\%) and GPs (71\%). Among the nurses and the doctors of the child health centers, use was dependent on whether they did meet with a parent who possessed an information booklet. Until now this situation has not occurred very often. Long term evaluation showed that more GPs handed out the complete parent booklet at one visit to parents of new patients, compared to the findings at the posttest. But, still the information was discussed with most of the parents. Again, most health care providers and parents interviewed expressed their appreciation of the asthma protocol and indicated their positive intention to start or to continue its use.

Health care providers' and parents' reports about cooperation with other disciplines revealed that joint care was supplied for only a fraction $(20 \%)$ of the children. However, this did not necessarily imply that the parents in this study received inadequate or incomplete patient education. Most modules were distributed and discussed. Furthermore, some actions such as referral to a community nurse for a check of the environmental factors in the home, were optional and may be more relevant for children with severe asthma and features of allergy than for mildly affected children. 
The impact evaluation tested the effectiveness of the education protocol in a randomized trial with a pre-and posttest of the two study groups mentioned earlier. Analysis of the behavioral variables revealed that the education protocol appeared to be an effective curriculum to favorably alter parents' knowledge, attitudes, perceptions of self-efficacy and self-management behavior. Level of state-anxiety minimally changed in both conditions. With respect to knowledge the greater improvement in the experimental group could be attributed mainly to modules dealing with how to apply asthma knowledge. The greater attitude change could be largely ascribed to items dealing with parents' beliefs about medication, indicating that parents changed towards a more adequate attitude to the benefits of using medication. The greater improvement in self-efficacy beliefs can be traced to a higher estimation of one's competence to take preventive actions and to treat an asthma attack. The improvement in self-management behavior occurred mainly for items on actions that could be taken to prevent an attack or to monitor the progress of an attack. Besides, this latter increase in the experimental group was more a result of a higher reported frequency of performance than of an increase in the number of different kinds of reported behaviors. Analysis showed that an increase in knowledge was the best predictor of gain in reported self-management behavior; gain in self-efficacy was the second best. The other variables did not contribute significantly to the prediction.

Twelve months after the posttest a follow-up study was conducted on the experimental group with respect to the same behavioral variables as measured at posttest. Because the (waiting list) control group received the educational materials after the posttest of the intervention, this group could no longer serve the purpose of a control group. Analyses of the data of the experimental group (28 GPs representing 46 patients) showed that the improvements, which were achieved at the posttest measurement on the behavioral variables knowledge, attitude, self-efficacy, and self-management behavior, were maintained at one year follow-up. Furthermore, it was revealed that the level of state-anxiety which parents experienced during their children's asthma attacks decreased in the experimental group.

Although the results achieved so far with the education protocol were encouraging, we still had to consider an important methodological issue. The conclusion of whether actual behavior changes occurred was limited because we had to rely on self-reports of parents. Especially treatment subjects may have felt more compelled to report 'appropriate' management behaviors than control subjects who did not receive an education intervention. Therefore, benefits in health outcomes needed to be and were studied as well.

To replace the waiting list control group (which could no longer serve as controls after the receipt of the parent booklets), a new control group was composed of asthmatic children who were similar to the children in the experimental group with regard to time of diagnosis and age. Medical care data from this new control group (18 GPs and 38 patients) were used to compare with findings of the experimental group. Data were collected from medical records on the period of one year preced- 
ing the pretest of the intervention and one year after the posttest of the intervention. Furthermore, several questions were posed to the GPs to reveal their impressions about the severity of the child's asthma symptoms before and after the intervention period. Medical care use data were collected on the number of emergency and non-emergency contacts with the GP, the number of telephone contacts, number of trips to the emergency room and/or trips to the specialist, hospital stays, perceived asthma severity and perceived duration of asthma symptoms. Analysis of the data showed that the number of visits to the physician's office or home visits of GPs (whether or not with urgence) and the number of hospital admissions decreased more over time in the experimental group compared to the new control group. Severity of asthma symptoms as judged by the GP, was reduced more over time in the experimental group, compared to the control group. Also, the perceived duration of attacks decreased more in the experimental group than in the control group.

\subsection{General discussion}

The main goal of the studies described in this thesis was to acquire insight in how an education protocol could be developed that addresses both the organization, the content, and the transfer concerning the asthma education in primary care. Three studies were conducted to develop the education protocol: a study to divide. educational tasks among disciplines involved, a study to reveal parents' needs and misconceptions about (the management of their child's) asthma and a study in which the protocol was pretested and subsequently revised. Another three studies were carried out to test whether the education protocol was effective when implemented in primary health care: a randomized trial to study the most important. behavioral effects, a follow-up study to research maintenance of the behavioral effects in the experimental group and a study in which changes in clinical outcomes were examined. This section focusses on general discussion of the studies' results with respect to the development and the implementation of the protocol, including a discussion of the methodology of the studies conducted. Furthermore, the implications from these studies for the development of education protocol are presented.

\subsubsection{The protocol's development}

Most children with asthma experience symptoms before the age of four. Furthermore, the majority of the children with asthma are treated in primary health care. A planned and systematic approach toward the asthma education by primary care providers seems necessary to teach parents of preschooi children with asthma how to manage this disease. This means that primary care providers need to know what part of the asthma education will be a disciplines' responsibility (organization), what information they should provide to parents of preschool children with asthma 
(content) and how they should provide education (transfer) to these parents. Education protocols have been suggested as an aid for care providers to optimize their educational practices.

The first study established the division of tasks among the disciplines involved. It was found that, although consensus about tasks is considered a prerequisite if different disciplines are expected to cooperate, methods on how to establish such an agreement were lacking. Therefore, a method to gather information on task conceptions and a technique to divide tasks were developed. The written questionnaire yielded information about care providers' task conceptions and knowledge about asthma. Subsequently, using the technique that was based on a discipline's claim, consensus with the other disciplines and the level of knowledge with respect to the task in question, we were able to divide all the tasks between the different disciplines. Furthermore, the revealed impression of the disciplines' moderate level of knowledge made us conclude that asthma information should be provided to the care providers in a detailed format. During the actual implementation of the protocol in primary care no difficulties were experienced that were related to the division of tasks. Therefore, we feel confident enough to conclude that our approach to the division of educational task has revealed a division that is manageable when implemented in a real life situation.

The study among parents was conducted because parents' interpretation of facts that relate to the management of their child's disease was considered crucial for the development of the education protocol. The study indeed revealed fundamental andinexpected bottlenecks and shortcomings in the provision of regular education in primary care. A very important finding was that parents experienced problems translating information into practice. The research findings were helpful in writing the content of the parent booklet in such a way that it closely related to the needs of parents. Although the focus group interviews and the written questionnaire appeared to be valuable research tools, they too have their limitations. The nature of focus group interviews significantly limits generalization to a larger population because results may be strongly dependent upon specific intra group interactions, and because respondents are not representative of the target population. Furthermore, the small number of respondents that participated in the focus groups and questionnaire limit generalization of results as well. However, with regard to the discovery of misconceptions and explanation of why and how parents manage asthma, the focus groups and the questionnaire do represent valuable research techniques. Based on the study findings a more general theory was used to link concepts as found in the focus group data to one another. Social Learning Theory appeared to be suitable for the situation of the management of asthma by parents.

The main purpose of the pilot study was to pretest the education protocol. In general, the pilot study showed positive changes among parents (from pre- to posttest) in knowledge, attitude, self-efficacy and self-management behavior. While the pilot study was clear in showing that changes in the desired direction occurred, the lack of a control group made results difficult to interpret. However, as a first step, this study was useful and provided the necessary legitimacy for implemen- 
tation in primary care and for further evaluation.

An important question is whether the instruments used to measure behavioral variables in the pilot study and the implementation study (which will be discussed hereafter), provided an accurate estimate of the education protocol's effects. A measure is considered reliable to the extent that in a given situation, it produces the same results repeatedly (Rossi \& Freeman, 1985). Repeated use of the same behavioral questionnaires (pilot study, randomized trial and follow-up study) revealed comparable scores. Another important issue deals with the validity of the behavioral questionnaires. Rossi and Freeman (1985) have formulated four criteria that are helpful in considering measurement validity. First, a concept to be measured, such as self-efficacy, should be used consistently with past work that has used that concept. It can be noted that the knowledge, the attitude, the self-efficacy, the selfmanagement and the state-anxiety questionnaires were derived from already existing instruments (respectively Parcel, Nader \& Tiernan, 1980; Creer, Wigal, Tobin, Snyder \& Winder, 1986; Kok, De Vries, Mudde \& Strecher, 1991; Kok et al., 1992, Barthelomew et al., 1991; Van der Ploeg, Defares \& Spielberger, 1980). Second, a valid measure should be internally consistent. With the exception of the attitude questionnaire, the internal consistencies of the measures used were satisfactory. Third, for measures such as knowledge, self-efficacy and attitude to be valid they should in fact predict self-management behavior. Of the behavioral variables, the knowledge and self-efficacy questionnaires clearly implied predictability in this respect. Fourth, a valid measure should be consistent with alternative measures that have been proposed. A positive indication for this type of validity is that the different measures used in our studies all led to the same conclusion, namely that the education protocol produced positive changes in the lives of asthmatic children and their families. Finally, it needs to be noted that all questionnaires were pretested for readability and uniform interpretation.

Some remarks should be made on the selection of subjects for both the pilot: study and the later intervention study. Participation in the both studies was based on voluntary cooperation of health care providers and parents. Interested GiPs mainly asked those parents to participate who had visited their office during the recruitment period. Because physicians' medical records were rarely categorized by disease and/or age so that 0-4 year-old asthma patients could be easily retrieved, there was no other option than to wait for an asthma patient to visit the physician's office. Thus, the opportunity to participate was offered by GPs to some parents but not to others (Rossi \& Freeman, 1985).

Whether the GPs have had a biased influence on the selection of patients is hard to say. All we know is that it was not easy for GPs to find a patient for the study because mostly they remembered an asthma patient only when this patient had visited their office. An indication for this idea came from the observation that most GPs registered patients in the autumn and winter months, a time in which more asthma patients have trouble with asthma. Our impression is that GPs did approach virtually all patients who met the criteria and who did vișit the physician's office because of asthma during the enrolment period. Yet, we cannot be sure about this. 


\subsubsection{The protocol's implementation}

During the development of the education protocol, the randomized trial functioned as the study in which the protocol's effectiveness should be demonstrated. In this trial, short-term behavioral effects of the protocol were evaluated. Random assignment of participants was applied to promote the internal validity in such a way that the only systematic difference between the two study groups was assumed to be the fact that they got education by means of the protocol or not. Another strong indicator for the protocol's responsibility for the reported behavioral changes is that both the pilot study and the randomized control experiment revealed similar posttest scores for the experimental group.

When evaluating program effects, it is important to study data on the process of implementation as well. The implementation study revealed that the degree and ways of the protocol's use by both GPs and parents (according to the guidelines in the protocol) were satisfactory. Use by care providers seems dependent on actual contact with patients, which was less for the employees of the organizations for home care. This finding indicates that for a broader study of 'referrals' more general practices in an organization's district should participate in the study to increase the chance of potential encounters of employees of organizations for home care with asthma patients.

When a program appears to be effective in changing health behavior, it becomes interesting to study whether the effects revealed will be maintained over time. In the study about the protocol's long-term effects on the behavioral variables, the lack of a control group might not be a serious shortcoming since the effect variables of the experimental and control group have been shown to differ significantly at posttest.

Finally, clinical findings became important in the evaluation of our asthma education protocol after it had produced significant behavioral effects and these effects were maintained over time. No significant clinical findings could have indicated that there was still a major flaw in either the behavioral skills being performed by the parents or in the regimen by which the child with asthma was treated (Creer, Wigal, Kotses \& Lewis, 1990). Furthermore, the more independently collected medical data have the added advantage that they are less suspect to invalidating influences from self-reported data. The clinical significance of the protocol was studied with a nonequivalent control group design. However, although we did not find differences between the two study groups on the variables measured at pretest, this design provides no guarantee that no other alternative explanations for the findings might exist. The evaluation of the results on the clinical outcome data indicated that the protocol had a favorable impact on medical consumption patterns of patients whose parents who had received the protocol on asthma.

As with most studies, generalization of the behavioral and clinical findings to populations outside the study population require careful consideration. It is difficult to estimate how adequately our samples represents the population of primary care 
providers and parents of children with asthma to which the results are to be generalized. But, because the study results were based on 'real' health care providers and parents in their natural setting and because we did not discover subgroups in our sample for whom the protocol worked better or worse, we have confidence in the appropriateness of the generalization of findings to asthma patients (0-4 years) and care providers in Dutch primary care. Another important consideration that should be addressed in assessing the external validity of an evaluation is whether the protocol is delivered in a way that can be replicated on a large scale. Whether the dissemination of the protocol can be continued on a larger scale by people other than the researcher remains to be examined.

The protocol, evaluated in the reported studies, is one of the few education programs that are designed for use in regular primary care and that has demonstrated long-term effects. It is the only existing program with a special focus on the problems of children with asthma in a very young age group. With the education protocol 'Asthma among children' quality of education is optimized by standardizing the education to a certain extent. Furthermore, continuity of education is addressed by providing and discussing information during several contacts. Continuity of education is also stimulated by regulating referral among disciplines. However, to establish continuity of care and education for the population of preschool children with asthma, the asthma education protocol needs to be implemented in primary care on a larger scale.

\subsubsection{Implications for education protocol development}

What guidelines can be derived for this thesis for others who want to develop an education protocol? Figure 1 provides a strategy for the development of an education protocol. In this thesis the development was started with a comparison of existing self-management programs on asthma. In the case that (effective) education programs do not exist an alternate scenario to determine the content of the protocol would have been to involve, in small scale research, a panel of experts (in our case for instance GPs, health educators, members of organization for home care, pulmonologists or pediatricians) and key people representing patients. Before the relevant health care providers for delivery of the protocol are identified, we must find out through which channel most patients can be reached and contacted on a. regular basis. in our study genera! practice and the regional cross association appeared to be the most important intervention channels. Next, educational tasks need to be divided among the identified disciplines responsible for providing information. The technique to divide tasks among disciplines "which is described in this thesis, appears helpful when an adequate division of tasks does not exist or when one has no insight in the knowledge the relevant disciplines have. The informational content of an education protocol must be based on an understanding of the target population. Involvement of the target population in defining the content is considered crucial to the protocol's effectiveness. Therefore, step four in the development 
Figure 1

A protocol to develop an education program

\author{
1
}

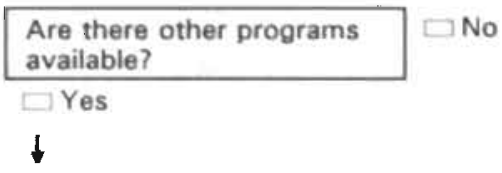

Are appropriately evaluated process, impact and health outcome data available?

$\square$ Yess

\section{$\downarrow$}

9

Is large scale dissemination

$\square$ No
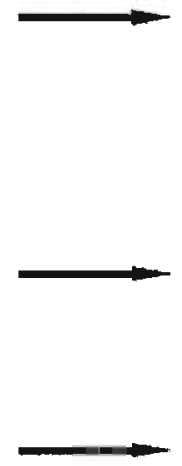

Determine best channel; identify relevant disciplines by representatives of patients: patient organizations, documentation, literature.

Determine task conceptions of disciplines involved; use technique to assign tasks.

Tailor content of the information to the needs, ideas and misconceptions by focus group interview.

Design the content and organization based on step 1-4; incorporate communication strategies found in literature.

Conduct pilot study:

- elfect evaluation

- judgement by representatives. of care providers involved

- pretest of content.

Check organizational structure in the disciplines work, look for opinion leaders, program coordinators.

Randomized controlled trial: collect behavioral data; clinical and health data; process data (use: how, extent, barriers).

Reveal failures. 
process focussed on tailoring the content with help of the target population. At step five the protocol can be designed. At this point a decision needs to be made with respect to the way the information will be provided. The next step concentrates on a small demonstration research study to test the protocol's efficacy. The protocol can then be modified (if necessary) and implemented in the setting for which it had been developed. Step 7 emphasized the importance of implementing a protocol in a way that links. up with the structure of the adopting organization (or person). Subsequently, process, impact and health outcome data should be assessed to estimate the effects of the protocol developed. Finally, protocols that have proven to be effective should be delivered to all of the intended target populations.

\subsubsection{Significance of education protocols}

Looking back at the development and implementation of the asthma education protocol it might be clear that using an education protocol assumes more from care providers than only handing out pages and/or discussing the pages in the parent booklet. Education protocols contribute to a process in which care providers become aware of their own ways of educating people and if necessary to change their routine for the better. The latter will contribute to the improvement of the quality of care.

At this moment, working with education protocois is not common practice among health care providers. We know that changing one's behavior is not easy. Therefore, it might not be expected that the education protocol will find easy reception with the relevant disciplines. For an effective implementation of the education protocol, a stable infrastructure is considered essential. In this respect it seems important to introduce existing and tested education protocols in formal training, in continuingeducation courses and in refresher courses intended for care providers who play a role in a particular protocol. For the asthma education protocol at this stage, the primary focus is the continuing education courses for GPs, community nurses, doctors in child health centers and the asthma nurses.

The development of education protocols follows naturally from the development of protocols for medical-technical care. To some people in the field, it may have appeared impossible to design a protocol for education about a chronic disease like asthma because of the numerous differences between patients. However, the research in this thesis shows that a general protocol is possible to some extent. On the other hand, an education protocol is simply an aid that gives health care providers something to hold on to; an aid that will standardize the information provided to some extent; and a guideline that can reduce inter-and intra discipline variation in the information provided. Nonetheless, checking the specific expectations, needs and concerns of patients remains, as before, indispensable for high quality education and treatment.

An education protocol is an operational description of the available knowledge at a certain moment in time. This implies that the asthma education protocol in 
future might need to be updated and adapted to changing times; recent medical and educational views should be incorporated when necessary.

\subsubsection{Final remarks}

This thesis described the development, the implementation and evaluation of a patient education protocol for the self-management of preschool asthma in primary care. In total four main studies were presented. One major conclusion of this thesis is that now an education protocol is available that is effective in helping care providers in enabling parents of preschool children to manage their child's asthma. Another major conclusion is that now guidelines are available on how to develop an education protocol that helps care providers to provide systematic and coordinated patient education.

The results described indicated that the national dissemination of the educational materials seems to be justified. This dissemination was initiated by the Dutch Asthma Foundation in January 1992 and is going to be evaluated by the Department of Health Education of the University of Limburg.

The actual dissemination will be the responsibility of the Asthma Foundation in cooperation with the asthma (staff) nurses (working in the organizations for home care). The asthma: (staff) nurses will receive a special training in the dissemination of the education protocol. For this purpose a conference was held in September 1992. It is not our aim to mail the education protocol to the relevant primary health care providers, but to introduce the protocol in person. Often these primary care providers will be the asthma staff nurses. Because a lot of primary care providers need to be approached, group meetings are preferred for this purpose. Per region of an organization for home care it will be checked whether the introduction of the protocol can take place during an already scheduled meeting, for instance a refresher course for GPs or community nurses, or whether such a meeting needs to be initiated. Decisions on the local level will determine which people are most suitable to initiate group meetings for GPs, community nurses and doctors of child health centers.

Two important events happened in the Netherlands that might make the nationa! dissemination of the protocol possible. Firstly, a new change was implemented recently in the organizations for home care that might favorably affect the implementation of the asthma education protocol. The fact is that organizations for home care have decided to increase the expertise of community nurses by establishing areas for special attention and subsequently assign specifically trained community nurses to these areas. Asthma is one of the first selected areas for special attention. Concretely, this means that some of the community nurses will be trained especially with respect to asthma care. The advantage of a specific asthma community nurse is that quality of care might increase. Furthermore, asthma patients who are referred to the organizations for home care will be handled by the (limited number of) trained community nurses, which means that the number of con- 
tacts with asthma patients during nurses' daily work will increase. It is expected that community nurses, who have chosen to work for the asthma care as their area for special attention, will be more motivated to use the asthma education protocol 1) because they meet these patients more often; and 2) they might therefore be more interested in asthma education materials than more general working nurses. An additional advantage is that the asthma staff nurse, who is responsible for the implementation of the asthma education protocol in the organizations for home care, only needs to introduce the education protocol to the community nurses who specialize in asthma. For instance, in an organization of average size, instead of an average of 80 community nurses, for instance only 7 asthma community nurses need to get familiarized with the protocol in a particular organization.

Secondly, in 1992 a medical protocol for children with asthma (0-4 years) was published in an authoritative journal for GPs in the Netherlands (Dirksen et al. 1992). The development of medical protocols was initiated by the Dutch College of General Practitioners. Together with some medical protocols, this organization also develops refresher course materials that they supply with help of the National Organization for General Practitioners to the latter organizations' regional coordinators. Subsequently, a regional coordinator organizes refresher courses for GPs. What does this mean for the asthma education protocol? In the publication mentioned earlier, a reference was made to the. Asthma Foundation as the address to request patient education materials. But, even more important, the parent booklet of the asthma education protocol will be included in the materials that are going to be used in the refresher course.

In future the pediatricians in hospitals will be informed about the education protocol. Although they see only $20 \%$ of the asthma patients it is helpful for them to know about the existence of the protocol as well, because they initiate most referrals to the community nurses for an adaptation of the patient's home.

Finally, besides these national events that will affect the dissemination of the education protocol beneficially, it is still necessary to check in each region in the Netherlands what the most appropriate channels for distribution of the asthma education protocol are. This will be the focus of a new study. 


\section{Multidisciplinary cooperation in primary care for asthmatic children}

Published, with some alterations, as: Mesters, I., Meertens, R., Mosterd, N. (1991). Multidisciplinary co-operation in primary care for asthmatic children. Social Science \& Medicine, 32, 65-70.

\section{Abstract}

In the development of education protocols for the treatment/education of chronic diseases in primary care the involvement of several disciplines of care providers must often be taken into account. This implies that tasks will have to be divided across several disciplines. An orderly way to do this does not seem to have been suggested in the literature. In this paper a systematic technique is presented to divide educational tasks across disciplines. The basic idea of this method is that one should link up as much as possible with existing task conceptions and knowledge of involved disciplines of care providers. The general description of the technique, will be followed by a piece of research in which the technique is applied to protocol development for parents of asthmatic children (0-4 years). The protocol aims at allocating specific asthma education tasks to community nurses, GPs, asthma nurses and doctors of child health centers in the Netherlands. This paper focusses mainly on results for community nurses.

Three criteria were considered in assigning tasks to the different care providers: whether the discipline in question claimed a certain task; whether the other three disciplines agreed with the claim, and whether the discipline in question had appropriate and sufficient knowledge about asthma to perform the task correctly. Community nurses did claim several tasks in asthma care, but knowledge did not always seem to be appropriate. Finally, some additional results are discussed. It appeared that nurses who had taken a refresher course did have a 
higher knowledge level than nurses who had not taken such a course. Regular contact with asthmatic children tended to promote nurses' knowledge on asthma too. The implications of these findings for the asthma education protocol are discussed.

\subsection{Introduction}

In view of the problems associated with the management of chronic diseases, an increasing number of patient education programs have been developed. The majority of these programs provide disease-related information through group education during a restricted period of time. These programs have had positive results, such as increasing patients' knowledge, decreasing medical consumption, ameliorating attitudes and behavior of patients (Clark et al., 1981; Lewis, Rachelefsky, Lewis, De la Sota, \& Kaplan, 1984; Creer \& Winder; 1986, Kaplan, Chadwick \& Schimmel, 1985; Paulozzi, Norman, McMahon \& Connell, 1984; Korhonen et al., 1983). However, a major disadvantage of group-oriented programs is that continuity of care/education, which is necessary for a long-term maintenance of effects, cannot be established through this approach. Education in individual patient-care giver contacts might not have this disadvantage, although a systematic approach of the education will be necessary to accomplish results which are comparable to group education programs. Therefore, more attention has recently been paid to the development of protocols for planned and systematic patient education programs to improve quality of care in patientcare giver contacts.

A protocol may be presented as a manual, a checklist, or a scenario. An education protocol often contains guidelines summarizing the educational approaches appropriate to a certain disease, promoting complete information and at the same time preventing provision of unnecessary and/or harmful information. In ali, a protocol is likely to identify the content of the patient information, the way in which the information should be provided to patients, and the disciplines which should provide which specific information. The content of the protocol and the way of transferring the information might often be deduced from existing gioup education programs. However, deciding who has to provide what part of the information is often a more problematic question to answer, because in primary care one must frequently take into account the involvement of several disciplines in the primary care of chronic patients. An analysis of the literature failed to reveal techniques for division of tasks across several disciplines in the protocol.

The purpose of this chapter is to present a systematic method for dividing tasks among disciplines of care providers. This division of tasks is supposed to be primarily a division of responsibilities, which should not be applied rigidly. $A$ general description of the technique is provided and illustrated by a study which was designed to develop a protocol for parents of asthmatic children, from birth 
to 4 years of age. In the study GPs, doctors at child health centers, asthma nurses and community nurses are regarded as relevant care providers in the primary asthma care. The focus will be on opinions of community nurses and the opinions of the remaining disciplines on the community nurses" tasks in the care for asthmatic children and their parents.

\subsection{A systematic way to go about the question "who does what"}

When care providers are expected to use the protocol, it is evident that they should (at least to some extent) agree with tasks assigned to them and to others. This is perhaps even more important when cooperation is required in the protocol. Research (Berg, Eckhoff-Biagi, Hebert, Rodell \& Sprafkin, 1987) has already shown the necessity of eliciting care providers' task conceptions to accomplish an efficient use of a protocol. Therefore, the protocol aims at relating the division of tasks among different disciplines as closely as possible to already existing task conceptions of the disciplines involved. Further, insight needs to be gained about care providers' knowledge with regard to rasks in the treatment/education of the chronic disease in question. When somebody claims a task, he/she should have the knowledge necessary to perform the task. Hence, information has to be collected about which tasks different disciplines claim with regard to a specific disease, the extent to which care providers agree with each others claims, and whether they have enough knowledge about the different tasks to perform them properly.

Table 1 provides an overview of possible alternatives in dividing tasks considering the above mentioned three criteria. In total, eight combinations can be distinguished. The consequences for the protocol, as described in Table 1, necessarily remain tentative because claims and knowledge of other disciplines involved must be taken into account too. The table shows a fairly simple method to assign tasks. As can be derived from this table, knowledge is not considered to be a major barrier for assignment, because a deficiency may usually be removed by training. However, the actual asșignment of tasks is mare complex. Combination $A$, for example, recommends assignment to the discipline in question because all the criteria are met. This does not exclude the possibility; though, that other disciplines might consider themselves equally responsible for the task, and that for them the criteria are likewise met. In such cases cooperation between both "competing" disciplines might be considered. Another important point might be whether a task corresponds to the other tasks of a discipline. If not, another discipline might be a more plausible candidate for this task (despite all criteria appearing to be met). Finally, task overload of one discipline must be avoided when assigning tasks.

The above described technique was used in the development of an education protocol for parents of asthmatic children (0-4 years). This protocol should regulate asthma education by community nurses, asthma nurses (staff nurses who 
have asthma as area for special attention), GPs and doctors of child health centers. As mentioned in the introduction, the description of the results will concentrate on community nurses. Before going into the results, however, the role community nurses play in Dutch primary health care requires some explanation. In the Netherlands community nurses perform tasks of both district nurses (curative work) and health visitors (preventive work). The nurses, working at local health centers, provide services for chronic patients in cooperation with other disciplines, for example with regard to chronically ill children with doctors

Table 1

Possible outcome-combinations based on three criteria

\begin{tabular}{|c|c|c|c|c|}
\hline Combination & $\begin{array}{l}\text { Task claimed } \\
\text { by one discipline } \\
\text { in question }\end{array}$ & $\begin{array}{l}\text { Agreement of } \\
\text { other discipline }\end{array}$ & $\begin{array}{l}\text { Knowledge of } \\
\text { the discipline } \\
\text { in question }\end{array}$ & $\begin{array}{l}\text { Implications for the } \\
\text { protocol }\end{array}$ \\
\hline A & yes & yes & yes & Consider assignment \\
\hline B & yes & yes & no & $\begin{array}{l}\text { Consider assignment and } \\
\text { augment knowledge } \\
\text { throught training and } \\
\text { giving information in the } \\
\text { protocol }\end{array}$ \\
\hline $\mathrm{C}$ & yes & no & yes & $\begin{array}{l}\text { Consider assignment } \\
\text { when cooperation will } \\
\text { not cause problems }\end{array}$ \\
\hline $\mathrm{D}$ & ves & no & no & $\begin{array}{l}\text { Consider assignment } \\
\text { when cooperation will } \\
\text { not cause problems, and } \\
\text { knowledge can be aug- } \\
\text { mented by giving infor- } \\
\text { mation in the protocol or } \\
\text { through training }\end{array}$ \\
\hline E & no & no & no & $\begin{array}{l}\text { Assignment not recom- } \\
\text { mended }\end{array}$ \\
\hline $\mathbf{F}$ & no & no & yes & $\begin{array}{l}\text { Assignment not recom-- } \\
\text { mended }\end{array}$ \\
\hline G & no & yes & no & $\begin{array}{l}\text { Assignment only recom- } \\
\text { mended after consulta- } \\
\text { tion with, and accept- } \\
\text { ance by the disciplines } \\
\text { in question }\end{array}$ \\
\hline$H$ & no & yes & yes & $\begin{array}{l}\text { Assignment only recom- } \\
\text { mended after consul- } \\
\text { tation with, and accept- } \\
\text { ance by the disciplines } \\
\text { in question }\end{array}$ \\
\hline
\end{tabular}


of child health centers.

\subsection{Technique applied to asthma}

\subsubsection{Asthma in young children}

In young children asthma appears to be the most important chronic disease (Lee, Winslow, Speight \& Hey, 1983; Newacheck, Budetti \& Halfon, 1986). Furthermore, it is known that the majority of asthmatic children show asthma symptoms before the age of four (Speight, Lee \& Hey, 1983). Early recognition and adequate treatment might prevent or reduce future asthma complaints (Kerrebijn et al., 1976). However, although nowadays adequate medication is readily available, a fairly large proportion of asthmatics are still not symptom free. So, it might be concluded that the management of asthma still poses many problems, which appropriate patient education might solve. Hence, the past few years have shown an increase in the number of health education programs for childhood asthma, especially in the United States (Maiman, Green, Gibson \& Mackenzie, 1979). However, these programs rarely involved several disciplines at once, as in this study.

\subsubsection{Method}

A questionnaire on task conceptions about asthma was developed in order to obtain the necessary data. At first an inventory, based on other asthma programs, was made of the information parents of asthmatic children need to manage their child's asthma. Thereupon, several tasks to provide parents with (asthma-) information were designed. In order to check validity, a draft of the questionnaire was evaluated by representatives of each participating discipline. Finally, 42 tasks in the care for asthmatics could be identified and clustered in nine major categories: (1) "the cooperation with other disciplines", (2) "noticing asthma symptoms" and (3) "other related problems", (4) "home adaptation", (5) "coordination of care", (6) "giving information on medication", (7) "providing asthma knowledge", (8) "general advises on asthma" and finally (8) "following updating courses".

In the questionnaire the community nurses were expected to indicate their conceptions of tasks in asthma care and treatment. Each question concerning task conceptions was followed by five response possibilities, of which four represented the disciplines earlier mentioned and a fifth represented any possible remaining discipline. Because more than one discipline might be considered responsible, the respondents were allowed to indicate more than one discipline on each single task. An example of the questions concerning task conceptions is 
given below:

Providing information to parents about triggers of an asthma attack.

This is the task of

the general practitioner

the community nurse

the doctor of child health centers

the asthma nurse

others, namely

Questions on knowledge (39 items) were formulated for several tasks to obtain an indication of care providers' ability to perform a certain task qua knowledge. As with the questions, on task conceptions the knowledge questions were evaluated by representatives of the different disciplines. SubsequentIy a pediatrition, specialized in lung diseases, was asked to judge the knowledge questions on medical relevance and accuracy. An example of these knowledge items is given below. A single answer had to be chosen out of five options, (including an "I don't know" option):

A person's peak-flow is:

$\square$ the same at every moment during the period of twenty-four hours

$\square$ lower in the morning

$\square$ lowest at noon

$\square$ mostly higher at night

$\square$ I don't know

\subsubsection{Sample}

Representatives of the four disciplines were selected as follows. A hundred GPS were randomly selected from the address file of the Netherlands Institute of Primary Health Care (NIVEL). This file contains data on virtually all GPs in the Netherlands. In order to represent the Dutch population of GPs as much as possible, the sample selection was stratified on type of practice (solo practice, paired practice, group practice and 'health center practices'). GPs in each category were selected in proportion to the distribution of type of practice in the overall population of GPs. The selected sample of GPs further resembled the overall sample with respect to age and degree of urbanization. Throughout the Netherlands (provincial), organizations for home care vere approached to act as mediator in approaching asthma nurses, community nurses and doctors in the child health centers for participation the survey. A contact person was assigned by every (provincial) organization to assist us in selecting the employees for study and in distributing the questionnaires among the picked employees. All available asthma nurses were requested by the contact person to fill oult a questionnaire. This was done because the absolute number of these nurses in the 
whole country was less than 100. Furthermore, the contact person selected the requested number of the two remaining disciplines from the employee address file by using an interval. For each organization the interval was obtained by dividing the total number of persons of one discipline by the number of respondents needed. This was done separately for the community nurses and the doctors of the child health centers. Finally, it needs to be noted that provinces with larger populations were represented by a proportionally larger number of community nurses and doctors in child health centers.

With each questionnaire, an instruction, introduction and postage paid return envelope were enclosed. The completed questionnaires could be returned anonymously. The time needed for filling in the questionnaire was estimated at 35 minutes. The data were gathered in the period June-September 1988.

\subsubsection{Data analysis}

Cronbach's alpha $(a)$ was used to measure the internal consistency of the items in the knowledge questionnaire. For every task, frequencies of the four disciplines' opinions were calculated. This means that four different tables could be obtained, representing the views of both asthma nurses, community nurses, doctors of child health centers and GPs on either the asthma nurses' tasks or the community nurses' tasks or the doctors of child health centers" tasks or the GP's' tasks (Appendix 3, Tables, 7-10). With tasks for which less than all four disciplines either scored above $66 \%$ or below $66 \%$, an additional statistical analysis was carried out to check whether this deviation among disciplines was significant. For this purpose the binomial test of homogeneity was employed (Van den Brink \& Koele, 1987). Finally, in Table 3 and Tables 4-6 (Appendix 3) the available frequencies per discipline are presented in a slightly different way than in Tables 7-10. Per table the opinion of a discipline in question on tasks: they consider relevant for their own discipline is presented together with the weighted opinions to this respect of the three remaining disciplines combined. Furthermore, it was indicated per task whether the knowledge of the discipline. in question was sufficient. Pearson correlations were calculated between leve! of knowledge and respectively the variables age, date of graduation, frequency of contact with asthmatic children and number of years occupied. Univariate analysis of variance (ANOVA) was performed to analyze differences between subgroups per discipline (taking a refresher course or nor) with respect to the knowledge score. 


\subsection{Results}

\subsubsection{Demographic data}

Table 2 shows the response rates per discipline. The mean age of community nurses in this study was 34 . The youngest nurse was 23 years of age and the oldest 59. Most respondents $(96 \%)$ were women. They all qualified between 1958 and 1987 (median $=1980$ ) and had been working as community nurses for at least 1 but not more than 24 years ( $M=6$ years). A fairly large number of the community nurses reported that they regularly encountered asthmatic children during their daily work. Only the community nurses were asked to answer this question. Doctors of child health centers see almost all 0-4 year olds in the Netherlands. Asthma nurses only meet asthma patients, and GPs will evidently encounter a child if it has asthma, but the community nurses might meet a child with asthma. The mean age of the GPs was 42 years (range 31 64 years). Most respondents were males (92.5\%). They all qualified between 1954 and 1983 (median = 1973) and had been working as GPs for at least 2 years, but no more than 34 years $(M=12.6$ years). GPs were working in solo practices $(51.5 \%)$, paired practices $(33.3 \%)$, group practices $(9.1 \%)$ or health center practices $(6.1 \%)$. The average size of a practice was $2001-3000$ clients. The mean age of the doctors of child heaith centers was 40 years (range $27-61$ years). Most respondents were women $(80.4 \%$ ). They all qualifled between 1952 and 1986 (median = 1974.). On average, they had worked 11 years as a doctor in the child health centers (range 1 - 32 years). Most asthma nurses were women $(87.5 \%)$. On average they were 44 years old (range 24 - 59 years). They had been working as an asthma nurse for 7 years on average (range 1 - 20). They all qualified between 1953 and 1988 (median 1973).

Table 2

Overview of response of disciplines involved

\begin{tabular}{lccc}
\hline Disciplines & Target number & Response & Selection \\
\hline Community nurses & 100 & $82 \%(N=82)$ & Via intermediaries \\
General practitioners & 100 & $66 \%(N=66)$ & Personally addressed \\
$\begin{array}{l}\text { Doctors of child health } \\
\text { centers }\end{array}$ & 100 & $51 \%(N=51)$ & Via intermediaries \\
Asthma nurses; & $58^{\circ}$ & $69 \%(\mathbb{N}=40)$ & Via intermediaries \\
\hline
\end{tabular}

Note. Maximum number of asthma nursesi available in the cooperating provinces in the Netherlands. 


\subsubsection{Task conceptions and knowledge}

Table 3 summarizes responsibilities of nurses as seen from the nurses' and other disciplines' point of view. In Table 3 the weighted opinions of GPs, asthma nurses and doctors of child health centers about community nurses' tasks are combined $(N=157)$. For several tasks no questions on knowledge were formulated, which is indicated in Table 3 by the term "not applicable" "Table 3 also gives information about the appropriateness of nurses ${ }^{*}$ knowledge. The figures in the second and third columns of the table indicate the number $(\%)$ of care providers which believe that the task in question is a community nurses' task. Tasks are regarded as claimed by and/or intended for community nurses (*) when about 2/3 of the respondents in both columns considered a task as belonging to the responsibility of community nurses. The discussion of the results will be limited to tasks claimed.

With regard to referring asthmatic children to other health care workers, community nurses believe it to be their task to bring in an asthma nurse in the care of a patient. Furthermore, the other disciplines agree with the nurses' claim. Although, doctors of child health centers and GPs consider themselves likewise. responsible, in the protocol this task is assigned to community nurses because this task corresponds closely to their other tasks.

Pointing out asthma problems at patients' homes and at the health center are considered to be tasks of community nurses. Because the first mentioned task is also claimed by GPs, cooperation between these disciplines might be considered. In like manner, the second task is equally claimed by community nurses and by doctors of child health centers. Because knowledge of both disciplines seems to be insufficient, training will be necessary for all the disciplines in. volved.

Pointing out other problems to parents, for instance with inadequate self care or the upbringing of children, is viewed as the responsibility of community nurses, a responsibility which may be shared with GPs and/or doctors of child health centers.

Examining children's homes to exclude any unfavorable circumstances with regard to asthma was considered by all disciplines to be a community nurse's task, if necessary assisted by an asthma nurse. However, community nurses' knowledge was inappropriate unlike that of the asthma nurses. Because the. asthma nurses' function is meant to be supportive of community nurses, community nurses will be well advised to ask an asthma nurse to come along the first time they are going to suggest adaptations of patient's homes. The community nurse and asthma nurse also claim to be important in providing parents with information concerning the management of adapted homes and the inevitable financial costs related with the adaptation of the home. Assignment of these tasks seems advisable though, since training of nurses will be necessary, especially for the first mentioned task. Although most nurses regarded themselves as contact person in the care for asthmatics and as intermediary 
Table 3

Community nurses 'and other disciplines' view on community nurses' conceptions, and related level of nurses knowledge

\begin{tabular}{lccc}
\hline & Nurses' & $\begin{array}{c}\text { Other } \\
\text { disciplines' } \\
\text { view } \\
\text { view }\end{array}$ & $\begin{array}{c}\text { Nurses' } \\
\text { knowledge }\end{array}$ \\
Area & Task & $(N=82)$ & $(N=157)$
\end{tabular}

Pointing out asthma related problems

Pointing out other problems

Adapting homes

Providing information about medication

Providing knowledge about

Providing genera! advice about

Referring an asthmatic patient to $a(n)$

Coordination of care

Refresher course during home visits

-at the child health center

-diagnosing asthma

-with regard to self care

- with regard to upbringing

-executing home visits

-advice on keeping the

home adapted

financial cost

-decision

-medication in general

-practice in the use of

medication

-side effects

-appliances

-appropriate use

-peak flow recordings

-monitoring therapy

-follow-up appointment

-during asthma attacks

-cause of asthma

-prognosis of asthma

-history of asthma

-social consequences

-asthma signals

-asthma triggers

-diagnosis

-consequences

-physical exercise.

-breathing exercise

-behavioral change

-via conservation groups

-(medical) services

-school selection

-tools (books etc.)

-specialist

-asthma nurses

-community nurses

-general practitioners

-pedagogue

-physiotherapist

-playing the central role

-being the contact person

-take a course $98.8^{\circ}$

$95.1^{\circ}$

0.0

$96.3^{*}$

$100.0^{\circ}$

$97.6^{*}$

$97.6^{\bullet}$

$72.0^{*}$

53.1

$62.2^{*}$

$90.2^{\bullet}$

28.8

55.0

34.0

12.5

30.9

28.4

3.7

$71.6^{\circ}$

54.3

53.1

$92.5^{\circ}$

$71.6^{*}$

$86.4^{\circ}$

1.2

$90.1^{*}$

$77.8^{*}$

32.5

$93.8^{*}$

$86.4^{*}$

$98.8^{\circ}$

44.0

$98.8^{\circ}$

4.9

$97.6^{*}$

24.4

52.4

37.0

14.6

$63.4^{*}$

$87.7^{*}$

$85.0^{\circ}$
96.5 - Insufficient

77.6. Insufficient

2.2' Insufficient

87.6." Insufficient

92.3" Insufficient

89.8" Insufficient

92.1 * Insufficient

$67.8^{\circ}$ Insufficient

38.1 Not applicable

45.9 Insufficient

78. $7^{\circ}$ Insufficient

18.3 Insufficient

50.0 Insufficient

37.2 Insufficient

15.1 Insufficient

26.3i Insufficient

21.2 Not applicable

2.7 Not applicable

65.8 - Sufficient:

52.3 Sufficient.

41.2. Sufficient

70.3 ${ }^{*}$ Insufficient

54.0. Insutficient

73.2. Insufficient

2.7 Not: appliçable

70.2 - Not applicable

$66.4^{*}$ Sufficient:

37.1 Sufficient

84.5 Insufficient

67.2 * Not applicable

84.5* Not applicab!e

37.6 Not applicable

93.6 - Not applicable

2.9 Not applicable

79.2. Not applicable

14.4 Not applicable

35.8 Not applicable

20.9 Not applicable

3.5 Sufficient:

$29.3 \quad$ Not applicable

53.9 not applicable

82.2 * Not applicable

Note. - Claim by approximately $2 / 3$ of the respondents of disciplines involved. 
between the disciplines and the patient, the other disciplines did not quite agree. Since, overall, GPs are considered to play the central role in the care for asthmatics and because these tasks are closely linked, assignment to the GP might be preferred.

With respect to tasks concerning practicing the use of medication, community nurses indicated they have an important role. The other disciplines agreed with this claim, but nurses will need training in this respect. Some nurses also considered it their task to provide parents with general information on medication, for example about the aim or order of intake. Assignment to nurses seems not advisable though, because their knowledge is inappropriate. Besides, a claim by another discipline is more evident and convenient, namely that by GPs.

Providing information about the cause of asthma to parents is considered by nurses as their task. The other disciplines agreed; moreover nurses' knowledge is appropriate. As this task corresponds to the tasks of GPs as well, it could be assigned to community nurses in cooperation with GPs. The same data pattern holds for assigning the provision of information on symptoms that indicate an asthma attack, although here training seems to be necessary, because appropriate knowledge is lacking.

Providing information about potential asthma triggers is considered to be a task for community nurses, but also for doctors of child health centers and GPs. Although nurses' knowledge is insufficient, assignment is recommended because it corresponds closely to the adaptation of homes. However, extra training will be necessary. Furthermore, cooperation with other disciplines is a possibility.

Informing parents about the possible consequences of asthma for the family (adapted vacations, removing pets from the home) is considered to be a task for nurses, asthma nurses and GPs. Each of these three disciplines could provide a part of this information on consequences of asthma. The community nurse and the GP claim to be equally responsible for providing parents with information on the social consequences of asthma (misconceptions and so on) though their knowledge is inappropriate. Both disciplines could cooperate in performing the task, but at first training seems necessary.

Furthermore, community nurses believe it to be part of their task to organize asthma conversation groups, and to advise parents on the use of tools and services. The other disciplines agreed with the community nurses ${ }^{\prime}$ claim. Therefore assignment of these tasks to the nurses seems to be appropriate. With regard to other aspects of asthma management, such as behavioral change (of the child) and physical exercise, nurses share responsibility with other disciplines. Especially with respect to 'behavioral change' further training of nurses seems to be necessary.

Finally, community nurses are in agreement with other disciplines in believing that they should take regular asthma refresher courses. However, of the responding community nurses, $40 \%$ had not taken a refresher course yet, whereas most of the others indicated that they had followed a course one or two years 
ago.

Besides making an inventory of task conceptions about asthma care the aim of this study was to gain some insight into which nurses have better knowledge on asthma. The reliability of the knowledge items appeared to be sufficiently high $(\alpha=82)$. Correlation of the variables age, date of graduation, taking a refresher course or not, and finally the number of years occupied as community nurse with actual knowledge level, as measured by the knowledge items in the questionnaire, yielded one significant result. As might be expected, community nurses who had taken a course scored higher on the knowledge items than community nurses who had not $(F(1,76)=7.03, p<.01)$. Furthermore, nurses in frequent contact with asthmatic children during their daily work scored somewhat better, but not significantly so, compared to nurses who were not $(F(1$, $76)=7.03, p<.06$ ). From a study of Maiman et al. (1979) it appeared that nurses who had experience with asthma themselves were better prepared as patient educators. Thus, experience with asthma by care providers (themselves or through patients contacts) might contribute to an improved quality of care. With respect to the (possible) relationships between demographic variables and the knowledge level of the remaining disciplines, the following can be reported. No significant findings were revealed for the doctors of the child health centers. As might be expected, asthma nurses who had taken a refresher course on asthma scored 'higher on the knowiedge items than asthma nurses who had not $(F(1,37)=5.31, p<.03)$. For the GPs, it was found that the younger the GP, the higher his score on the knowledge questionnaire $(r=-.37 ; p<.01)$; the fewer years the GP had worked or the more recent the GP was graduated (these variables are related), the higher the knowledge score of the GPs, respectively $r=-.31 ; \mathrm{p}<.02$ and $r=.33 ; p<.01$. Also GPs who had taken an refresher course on asthma had a higher knowledge score than those GPs who had not $(F(1,63)=5.20 ; p<.03)$.

\subsection{Conclusion}

The approach described here seems to be a fairly useful method of gathering information on health care workers' task conceptions and knowledge about asthma in order to establish minimal, but essential conditions for cooperation and thus for an integrated educational provision.

From this study one further might conclude that nurses play a relevant role in asthma care. Nurses claimed a considerable number of tasks, and there appeared to be considerable agreement among the other disciplines in this respect. These results suggest that other disciplines might leave these specific tasks completely to community nurses and as a result refer patients to them for these parts of the education.

In all, lacunae seem to exist in the community nurses' knowledge about asthma, but this finding also applies to a lesser extent to the other disciplines. In 
general, disciplines who had taken a refresher course on asthma performed better on the knowledge questionnaire. Overall, more training on asthma would therefore seem to be profitable. The existing gaps in knowledge make it clear that the protocol cannot be limited to checklists. At firsî, a more detailed manual is necessary to equip the disciplines with the essential knowledge to provide appropriate education. Only when the disciplines have mastered the asthma education, might checklists be used. The developed manual could then further serve as a reference book. 



\title{
3 Pediatric asthma, a qualitative and quantitative approach to needs assessment
}

Published, with some alterations, as: Mesters, I., Pieterse, M. \& Meertens, R. (1991). Pediatric asthma, a qualitative and quantitative approach to needs as: sessment. Patient Education \& Counseling, 17, 23-34.

\begin{abstract}
This article describes an approach to needs assessment in a specific location using both focus group interviews and a written questionnaire. In this case study, the target population consists of parents of asthmatic children ( $0-4$ years of age). Six focus group interviews were conducted with the purpose of discussing parents' experiences with health care for asthma, their beliefs and knowledge of general aspects of́ asthma, medication and preventive actions. As a supplement, parents completecj a written questionnaire providing additional quantitative information on parental knowledge and misconceptions. Results indicated that parental knowledge of asthma in general was not adequate, especially with respect to medication, preceding signs and preventive activities. Important misconceptions, which might contribute to noncompliance with self-management behaviors, were revealed during discussions with the parents. Several differences were found between results of the intervievvs and the questionnaire for instance regarding parental knowledge about preventive measures, medication and perceived parental satisfaction with health care for asthma. These differences indicate the value of using both a survey and a focus group.
\end{abstract}




\subsection{Introduction}

When designing asthma education programs or protocols it is clear in general what knowledge and skills are needed to manage the disease adequately. Such programs or protocols should be fitted closely to the already existing knowledge, concerns, needs and perhaps misconceptions of the target population. Information about such aspects can be gathered by means of a simple questionnaire. However, a common disadvantage of gathering data with a structured questionnaire is that important issues (like misconceptions) cannot always be foreseen, and consequently are not included in the questionnaire. Thus, techniques that allow a freer response mode, like group interviews, might serve as a complementary method in assessing educational needs for the target population.

This article describes an approach to needs assessment in a specific location using both focus group interviews and a structured questionnaire. The procedure of using combined qualitative and quantitative methods is illustrated via a case study aimed at gaining insight in the knowledge and, even more important, the lack. of knowledge and misconceptions of parents of young children with asthma. Attention was specifically paid to parents ${ }^{*}$ knowledge of essential aspects of asthma management, such as signs preceding asthma attacks, preventive actions, and the use of medicines. A second objective was to gain insights into the experiences and satisfaction parents have had with the health care provided by general practitioners. A further objective was to compare information obtained from both methods and to determine the potential advantage of the combined use of focus group and questionnaire methods.

\subsection{Childhood asthma}

Childhood asthma is a common respiratory disease characterized by intermittent or chronic, usually reversible airway obstruction. Studies in the Netherlands report that in the $0-4$ year age group approximately 8 per 1000 people have a diagnosis of asthma (Lamberts, 1984; Van den Hoogen, Huygen, Schellekens, Straat \& Van der Velden, 1985), though there are difficulties over diagnosis (Huygen et al., 1977; Kaptein, Dekker, Gill \& Van der Waart, 1987). The use of health care services as a consequence of asthma symptoms is considered extensive. It is estimated that 5 to $10 \%$ of children in the Netherlands need medica! treatment for their asthma symptoms (Van Mölken, Doorslaer \& Rutten, 1989). In 1987 asthma accounted for $1.7 \%$ of all hospitalizations for children aged 0.4 years (Centraal Bureau voor Statistiek, 1989a).

As with other chronic diseases, patient education is a critical factor in management of asthma. Studies to date have shown interventions to be successful, demonstrating improved self-management (Parcel et al., 1980; Lewis et al., 1984; Clark, Feldman, Evans, Duzey et al., 1986), reduced wheezing (Creer, Renne \& Christian, 1976; Evans, Clark et al., 1987), improved school attendance/adjust- 
ment (Creer et: al., 1976; Hindi-Alexander \& Cropp. 1984; Evans, Clark et al., 1987), improved family ability to cope (Parcel et al., 1980; Lewis et al., 1984; Clark, Feldman, Evans, Duzey et al., 1986), and finally changes in the use of health services, such as decreased emergency room visits and hospitalizations (Lewis et al., 1984; Hindi-Alexander \& Cropp, 1984; Clark, Feldman, Evans, Levison et al., 1986).

\subsection{Focus group interview}

The focus group interview, a qualitative approach originating from marketing research, seems to be a suitable method of gaining insight into the parents' views on asthma. A focus group interview is a discussion under the guidance of a moderator, in which a small number of respondents (6 to 12), chosen from some specific target group, talk about topics that are believed to be of special importance to the investigation (Folch-Lyon \& Trost, 1981).

Furthermore, focus groups are composed of people wha do not know one another and who are similar to each other on aspects determined by the purpose of the study (Krueger, 1988).

Traditionally, health education practice has used small group discussions as a method for program delivery or for skills training (Basch, 1987). However, more recently focus group interviews have proven to be a useful research technique (Heinmann-Ratain, Hanson \& Peregoy, 1985; Keller, Sliepcevich, Vitello, Lacey \& Wright, 1987). The major advantages of focus group interviews are the relatively low costs and the high speed with which data can be obtained, when compared to individual interviews (Fern, 1982).

However, some limitations can be distinguished as well. Data collected through focus group interviews are not necessarily generalizable: to a larger population, duei to possible selection bias as a result of setting, topic or interview method. Furthermore the data should be interpreted with great care, considering the inevitable personal biases of the moderator.

\subsection{Methods}

\subsubsection{The questionnaire}

\subsubsection{Instrument}

The questionnaire consisted of three major parts, the first part containing demographic items concerning parents' level of education, age and sex, and medical characteristics of the children, such as the type and severity of the symptoms. The second part focussed on parents' knowledge and possible 
misconceptions regarding prevention and treatment. Parents were asked to indicate. whether they agreed with statements, concerning basic facts about asthma, signs, preceding an attack, prevention of asthma symptoms and finally the use of medicines. All these statements could be answered with yes or no, yes indicating that they were known or performed by the parents. In each list of statements a few unnecessary or even adverse actions (as judged by a pediatrician) were included, to reveal misconceptions held by parents. The questions used were comparable to the questions asked in a study of Spykerboer et al. (1986). In the third part of the questionnaire the participants were asked to indicate to what extent they were satisfied with the care provided by their general practitioner. For this purpose four multiple choice questions were formulated, asking parents' satisfaction with the overall care, the time spent by the care giver, the information given on the causes of asthma and the explanation of treatment and prevention. procedures.

\section{4. 1.2 Data collection}

The questionnaire was completed by the parents at the start of the meeting to avoid any confounding effects by the group discussion. Immediately after the introduction by the moderator, the parents were asked to fill in the questionnaire, which took 20 to 30 minutes. It was designed to add some quantitative data to the results of the interviews.

\subsection{3 Data analysis}

Simple frequencies were calculated for the response of each question. Furthermore, the occurrences of correctly answered questions per topic were counted per parent for items that were related to asthma and for the unrelated (false) ones separately.

\subsubsection{The focus group interview}

Six focus group interviews were conducted, the smallest attended by 4 parents and the largest by 11 , resulting in a total of 42 participants. The interviews were located in three cities in the Netherlands. The participants were recruited through advertisements in local newspapers $(n=38)$ and an office of a local pediatrician ( $n=4)$.

\subsubsection{Instrument}

The group discussion was directed by using a semi-structured interview guide containing a list of anchor questions (Glik, Gordon, Ward, Kouame \& Guessan, 1988). These anchor questions were used to introduce new topics during the interview, after which more detailed sub-topics could come up. To obtain a list of 
anchor questions, literature was studied and problems or topics that could be associated with having a child with asthma were discussed with experts and parents of children with asthma. A range of possible questions and variations in phrasing were gathered as exhaustive as possible. When the list was complete and no further suggestions could be made, the critical questions were highlighted. These were the questions that captured the intent of the study (Krueger, 1988).

The structure of the results section is based on the written questionnaire, starting with a short description of the characteristics of the parents and their children. The results of the questionnaire and the focus group interviews are then combined for each separate aspect mentioned above.

\subsubsection{Data collection}

After the initial acquaintance of the participants, the aim and procedure of the meeting were explained by the moderator, an experienced group discussion leacier, who was well informed about the objectives of the research and about childhood asthma. Subsequently the interview was conducted, lasting approximately 90 minutes. In the last 30 minutes of the meeting the participants had the opportunity to pose questions to an asthma expert, present at each meeting.

\subsubsection{Data analysis}

The sessions were tape recorded with the consent of the participants. Subsequently the tape recordings were transcribed for the analysis. The tapes and transcripts have been studied separately by two researchers. The framework of the analysis (and report) of the focus group findings was composed of the anchor questions that were asked. Each researcher noted the most important aspects with every question. Special attention was paid to elicit misconceptions (and the way they arise) and important gaps in knowledge, because these aspects are amenable to education. The notes of one researcher well matched the notes of the other one, although some useful additions, could be made to each. Data were analyzed within groups and finally the response of the different groups were combined. A pediatrician, specialized in lung diseases, was asked to judge revealed misconceptions on medical irrelevance and inaccuracy. Findings are reported by a summary description with illustrative quotes, followed by a section (in the discussion) on what the data mean for the development of an asthma education program (Krueger, 1988).

The structure of the results section is based on the written questionnaire, starting with a short description of the characteristics of the parents and their children. The results of the questionnaire and the focus group interviews are then combined for each separate aspect mentioned. 


\subsection{Results}

\subsubsection{Subjects}

The 42 parents involved in the focus group interview represented 26 children, most of them aged between 1 and 6 years, with the average being 5 years. Virtually all of these children's asthma symptoms started before the age of $3(n=23)$, and in ten of these children already within the first twelve months after birth.

No standard methods are available to classify asthma as mild, moderate or severe (Creer \&. Winder, 1986). Nevertheless some questions were asked about the sort of symptoms the children suffered and with what frequency, to get at least a general impression of the type and severity of the asthma symptoms. Almost. every child suffered from wheezing, coughing, catching colds and trouble with sputum. Other symptoms, like sniffling, were reported somewhat less often. Many children were reported to catch bacterial infections with some regularity, while only 9 children were reported to catch viral infections.

Eczema occurred in $35 \%$ of these children, which is more or less comparable with results ( 11 to $41 \%$ ) of other studies (McNichol \& Williams, 1973; Fergusson, Horwood \& Shannon, 1983).

In all, 38 questionnaires were completed by the parents, 12 fathers and 26 mothers, in the age from 23 to 42 years $(M=33$ years). For each family at least one parent completed the questionnaire.

The average level of education of the participants appeared to be rather high, with an underrepresentation of parents with only elementary education.

In the next sec;tions, parents' knowledge and misconceptions about asthma and their experiences and perceived satisfaction with the care are presented.

\subsubsection{Prevention}

The questionnaire revealed that, on average parents knew a little more than half of the 18 beneficial actions (range 2 to 16). Furthermore, they held some important misconceptions as well. Analysis of the total number of misconceptions per case, showed an average of 1.5 misconception per parent (range 1-5). About 1/4 of the 38 parents considered letting the child sleep near a wide open window or keeping the indoor air moist as preventive actions; a minority of the parents $(11 \%)$ believed that replacement of flowers by dried flowers would be an appropriate action and approximately one third of the parents considered 'natural' furnishing as a beneficial practice. Because these factors may actually aggravate asthma symptoms they should be regarded as misconceptions.

Having good sanitary facilities, which is not related to asthma symptoms, were considered as a preventive action by $61 \%$ of the parents.

Moreover, beneficial actions like the placement of registers in the living room and 
bedroom (ventilation), usage of fabrics washable at high temperatures (to eliminate mites) and vacuuming the carpets to reduce dust, were subscribed to by $34 \%$, $53 \%$ and $76 \%$ of the parents, respectively. Other beneficial actions, such as removing triggers, cleaning the house to reduce triggers and furnishing the home with substances posing no allergic danger were known by more than $82 \%$ of the parents. Most parents $(92 \%)$ reported giving medications as a way to prevent symptoms, for example during a cold $(71 \%)$, before contact with a trigger $(61 \%)$ and in moist weather $(50 \%)$. When parents noticed overtiredness or breathlessness they restricted their childs' activities (68\% and $63 \%$, respectively). Several parents indicated that they avoided eating certain food products $(58 \%)$ or meeting people having flu $(34 \%)$. The majority of the parents were aware of actions, such as coughing up sputum $(79 \%)$, relaxation $(87 \%)$ and breathing exercises $(66 \%)$.

During the interviews parents were asked which actions they normally undertook to prevent their child from getting asthma symptoms, how they got acquainted with these actions and what difficulties they experienced when performing preventive actions. In each group session parents reported several preventive actions, confirming actions already mentioned above in the section about results of the questionnaire.

Parents in different groups shared the impression that information from their care providers about prevention was available, but mainly on their own initiative. Usually parents stated that they got acquainted with actions to prevent asthma symptoms by their own experience, reading written information or advice from other people who were familiar with asthma. The following is an example of frequently made statements: "You have to find it out yourself. It is up to you to get the information you need".

The issue of difficulties parents experience when taking preventive actions was expressed in different categories: lack of cooperation from other people (especially friends, family and schooll) and lack of specific knowledge about which, why, when and how actions should be performed. Illustrative quotes expressing parents' views about perceived difficulties: "After a while friends stay away if you asked them not to smoke." "I have been suffering from severe overstrain because I kept cleaning my home all day long. I just didn't know what was enough." "They told me I should prevent my child from getting out of breath, but I don't know exactly when you have to stop him."

\subsubsection{Medication}

Parents" knowledge was; assessed in the questionnaire about the administration of medicines when using the aerosol and the powder inhaler. These two appliances are especially important because they require proper instructions and skills training.

The questions contained five possible answers of which two were correct. The. two instructions for use of an aerosol that should be followed (Table 1) were mentioned only by half the parents whose children use an aerosol. The most 
frequently mentioned false one was "holding the aerosol bottom-down when inhaling". Analysis of the total number of correct instructions known or performed per parent, showed that on average parents answered less than 1 instruction correctly (range 0 - 2). Only 13 people agreed with at least one misconception (range 1 - 3).

For use of the powder inhaler, a similar list of instructions was set up. The correct procedures are subscribed to by less then half of the parents using a powder inhaler. The other three (false) instructions were mentioned by a minority of the parents. Again on average parents had less than one instruction answered correctly (range 0 - 2). Eight parents agreed to 1 or 2 misconceptions (range 1 2).

Table 1

Knowledge about medication instruction

\begin{tabular}{|c|c|c|c|}
\hline $\begin{array}{l}\text { Aerosol } \\
(n=32)\end{array}$ & $\begin{array}{l}\text { Frequency } \\
\qquad(\%)\end{array}$ & $\begin{array}{l}\text { Dry powder inhaler } \\
(n=29)\end{array}$ & $\begin{array}{r}\text { Frequency } \\
(\%)\end{array}$ \\
\hline Shaking inhaler before use (c) & $18(56)$ & $\begin{array}{l}\text { Inhale as fast. and deeply as } \\
\text { possible (c) }\end{array}$ & $13(45)$ \\
\hline $\begin{array}{l}\text { Inhale as slowly and deeply } \\
\text { as possible (c) }\end{array}$ & $15(47)$ & $\begin{array}{l}\text { Hunching one's shoulders } \\
\text { when inhaling (f) }\end{array}$ & $6(21)$ \\
\hline $\begin{array}{l}\text { Inhale deeply before actu- } \\
\text { ation of the aerosol (f) }\end{array}$ & $5(16)$ & $\begin{array}{l}\text { Need to inhale the powder } \\
\text { in one puff (f) }\end{array}$ & $6(21)$ \\
\hline $\begin{array}{l}\text { Holding aerosol bottom-down } \\
\text { when inhaling (f) }\end{array}$ & $9(28)$ & $\begin{array}{l}\text { Holding breath for } 10 \mathrm{sec} \text { - } \\
\text { onds after inhalation (c) }\end{array}$ & $12(41)$ \\
\hline $\begin{array}{l}\text { At least three puffs at a time } \\
\text { (f) }\end{array}$ & $2(6)$ & Storage in refrigerator $(f)$ & $0(0)$ \\
\hline
\end{tabular}

Note. (c) $=$ correct; $(f)=$ false

While the questionnaire provided data concerning the use of the powder inhaler and the aerosol, the interviews dealt with the form of medication children were taking, perceived effectiveness of the medication, how parents felt about their child taking medication and reasons for noncompliance with prescribed medical regimen. Besides powder inhalers and aerosols, capsules, tablets and liquids were used.

Within several groups parents appeared to be confused about medication effectiveness. Preventive medication, for example, was considered ineffective to some extent, because children still suffered from asthma symptoms now and then. When asthma attacks get more severe young children might have problems using inhalers adequately (for instance because of an inability to take a deep breath), meaning that the drug won't reach it's destination. Using other forms of medication (such as liquid forms), might be more effective under these circum- 
stances. When describing similar situations, parents stated that they doubted the effectiveness of the drug, instead of the medication form. This perception is illustrated by the following statement: "Just when we expected most of the drug. it didn't work."

The discussions about parental attitudes to asthma medicines and medication compliance were dominated by anxiety for negative physical consequences of the use of medication in general and especially for extensive, long term use of medicines. Anxiety was expressed in several ways. Parents were concerned about long term use of steroid medicines, which are known to retard growth. Also, many parents believed the asthma medicines were addictive, and were concerned that their child would get used to them and would need more and more. Inquiries about how these two misconceptions were established revealed the following argumentation: parents were disappointed when the amount of medication was increased in spite of all their efforts. But, in general, asthma medication is prescribed per kilo weight of the child, so doses are more likely to increase due to the relatively fast growth of young children. Parents, unaware of the relation between dose and weight, thus developed their own explanation for the increase of the amount of medication. The following statements illustrate views of the parents: "Every time the amount of medication increases I wonder how long it will take before we reach the maximum. Then we can't do anything anymore to remedy attacks." And: "I don't want my child to get addicted, he is only a baby."

Parents reported several situations in which they experienced negative physical outcomes when giving medication. Within several groups some parents mentioned short term side effects, such as dizziness and nausea. Anxiety for the use of medicines increased whenever parents thought that their physicians tended to minimize the possible occurrence and impact of side-effects. As a result parents felt even more responsible to watch for side effects themselves. An impression of parents' feelings can be illustrated by following quotes: "When I noticed dizziness I first reduced the dose but finally decided to quit the therapy altogether." "Who would check for side-effects otherwise, pharmaceutical industries do not say something negative about their products iff they don't have to."

A tendency to avoid (extensive) use of asthma medicines was revealed, indicated by expressions about delaying the use of the medicines as long as possible and the considerable number of alternative treatments parents tried.

As some parents indicated: "Instead of suppressing the symptoms with medicines I believe it is better to let the symptoms come out and to let the child get used to the symptoms: How else can a child grow out of asthma."

Several statements of parents dealt with the feeling of not being involved and acknowledged in making decisions about medication regimens. Sometimes this resulted in an argument with care providers: "Being at the emergency room we had a big discussion with the pediatrician who insisted on treating our child with a medicine of which we knew from prior experience that our child wouldn't respond to in the expected way." 
Finally, several parents expressed that it is difficult to administer medication to small children because they are to young to comprehend why and how to cooperate. Interesting is that, in view of this problem, few parents who used an aerosol were acquainted with special devices, such as Nebuhalers or Volumatics, which might have facilitated the use of aerosols.

\subsubsection{General aspects of asthma}

The parents appeared to be fairly well informed about the aetiology of asthma. On average parents knew three out of the four correct items. A high percentage of the parents agreed that allergic triggers (100\%) and infections $(84 \%)$ have a role in asthma. Emotions and exercise were known by about $60 \%$ of the parents. Factors, not related to asthma, such as weak health were still confirmed by $24 \%$ of the parents, but eating raw foods was not considered by any parent as a factor that can trigger asthma symptoms.

During the interview triggers were frequently mentioned in connection with questions about how to remedy or to prevent an asthma attack. Besides triggers included in the questionnaire parents frequently mentioned irritants (such as tobacco smoke, air pollution, and moisture) and stressed foods as important asthma triggers. Furthermore, it appeared from the interviews that many parents could not distinguish allergy from hyperactivity and bacterial from viral infections. Following statements illustrate examples of asthma triggers mentioned by parents: "When grandad is home they keep on playing and our child gets overexcited. I think he is sensitive to stress." "After our child had eaten peanut butter all the mucous membranes in his face got swollen. He looked monstrous within five minutes."

A second important aspect of asthma for parents is the ability to foresee a coming attack, which requires knowledge of preceding signs or symptoms. A list of 15 signs was given, including a few symptoms not related to asthma (Table 2). On average parents recognized at about half of the 12 correct signs (range 2 - 10). Very few, if any, parents subscribed to the misconceptions represented in Table 2. During the interviews parents were asked to talk about signs indicating that symptoms will start or are getting worse. The interviews in the focus groups supported the findings of few misconceptions regarding warning signs of an asthma attack. However most signs were associated with a later stage in the asthma attack. Signs were not considered as cues to action when the symptoms were mild or moderate, but most parents take actions when the symptoms interfere with their child's activities. For example: "Our child looks pale and his hands and fingers turn blue, he redraws himself." "Our child aiways gets very restlessness." "Shortness of breath, coughing and wheezing." "Whenever our child catches a cold or has a fever, I know an attack is on it's way." "He starts to cough and has a running nose." 
Table 2

Parental $(N=38)$ knowledge about symptoms

\begin{tabular}{|c|c|c|c|}
\hline Symptoms & $\begin{array}{r}\text { Frequency } \\
\qquad(\%)\end{array}$ & Symptoms & $\begin{array}{r}\text { Frequency } \\
\qquad(\%)\end{array}$ \\
\hline Cough & $33(87)$ & Stomach age & $9(24)$ \\
\hline Fast breathing & $28(74)$ & Hunched shoulders & $28(74)$ \\
\hline Diarrhoea * & $1(3)$ & Out of breath & $28(74)$ \\
\hline Pink colored tongue ${ }^{*}$ & $1(3)$ & $\begin{array}{l}\text { Blue fingernails and } \\
\text { lips }\end{array}$ & $11(29)$ \\
\hline Wheeze & $38(100)$ & Aggravated eczema & $9(24)$ \\
\hline Pain chest & $12(32)$ & Swollen stomach * & $1(3)$ \\
\hline Aching feet ${ }^{\bullet}$ & $0(0)$ & Bad breath & $17(45)$ \\
\hline Yellow colored skin ${ }^{\bullet}$ & $3(8)$ & More hungry* & $1(3)$ \\
\hline Stop activities & $18(47)$ & & \\
\hline
\end{tabular}

Note, - Misconceptions

In the questionnaire parents were asked to indicate which actions they undertake in order to remedy asthma symptoms. Analysis of the average number of actions taken revealed that parents knew almost five adequate actions out of the total number of seven mentioned in Table 3 (range 0 - 7). The total number of misconceptions averages out at 1 per parent (range 1 - 3).

During the interview comparable questions were asked about treating an asthma attack as with preventive measures. Parents experienced very similar problems as with taking preventive actions, namely perceived unavailability of information, lack of specific guidelines for what to do, and additionally lack of criteria to estimate severity of symptoms.

Furthermore, parents were asked whether they believed that their child would grow out of asthma. It appeared that many parents had been told or thought that their child would probably grow out of asthma, which is not true, strictly speaking: though symptoms may disappear temporarily the asthmatic disposition will remain (Spykerboer et al., 1986). Typical statements to this respect: "I think he will grow out of it. But actually, I don't know" and "Our doctor told me she will outgrow asthma, that's what he said." 
Table 3

Parental (N = 38) knowledge about actions during asthma episodes

\begin{tabular}{lr}
\hline Action & Frequency \\
& $(\%)$ \\
\hline Give medication & $34(90)$ \\
Submerge child in cold water * & $0(0)$ \\
Give drinks & $16(42)$ \\
Rest or restriction on child's activities & $32(84)$ \\
relaxation exercise & $25(66)$ \\
Toughen child by confronting it with the trigger * & $1(3)$ \\
Rub child's chest & $24(63)$ \\
Remove trigger from child & $30(79)$ \\
Home remedies * (herbs etc.) & $7(18)$ \\
Rest child in special position & $27(71)$ \\
Clothe child warmly* & $9(24)$ \\
Breathing exercise & $24(63)$ \\
\hline
\end{tabular}

Note. Misconceptions

3.5.5 Perceived experiences and satisfaction with the provided care

To assess parents' satisfaction with the care provided by the GP four items were formulated in the questionnaire. Since all children $(N=26)$ were represented by the mother, the data regarding parents' experiences and satisfaction with the care providers was limited to analysis of the mothers' questionnaires only, because here responses (of both parents) may be expected to be highly correlated.

In the questionnaire parents could list the number of disciplines they had consulted for their child's asthma. On average parents stated having consulted approximately three different disciplines, nearly always $(84.6 \%)$ including the GP. According to Table 4 less than half the parents indicated they were satisfied with their GP. Satisfaction could be expressed in the arnount of time GPs spent on explaining management procedures, the clarity of information provided, overall asthma care and whether parents thought that GPs took a serious approach to explain issues to them.

During the focus group interview parents were asked to which care giver they went first in connection with their child's disease; and what did the care giver do? Were other disciplines involved, and what did they do about the child's symptoms? Finally parents were asked to give an impression of how things had been going in terms of degree of satisfaction with care and education provided. 
Table 4

Parental knowledge $(N=26)$ perceived satisfaction with provided care from the general practitioner

\begin{tabular}{lccc}
\hline Statements & Positive $(\%)$ & Negative $(\%)$ & $?(\%)$ \\
\hline Overall asthma management & $10(39)$ & $10(39)$ & $6(23)$ \\
Time spent on management & $12(46)$ & $8(31)$ & $6(23)$ \\
Clarity of information & $13(50)$ & $6(23)$ & $7(27)$ \\
Seriousness of approach & $8(31)$ & $9(35)$ & $9(35)$ \\
\hline
\end{tabular}

In contrast to the questionnaire, more parents expressed themselves negatively with regard to both quantity and quality of received care and information by their GP. However, negative feelings turned out to be associated with the early stage of asthma. Most people first contacted their GP. They found themselves confronted with a GP, who at first did not appear to take the symptoms seriously and who tried to assure them that treatment was not necessary. From the parents point of view these GPs often hesitated to diagnose asthma in the first place, and more than once started treating the patient with antibiotics. At this stage some parents felt deserted by their GP, who appeared to trivialize their problems. Some parents experienced difficulties getting a referral to a specialist, in spite of the problems with the treatment. A consequence of these problems with the GP was that some parents were reluctant to contact the GP at night, even when symptoms were very severe. Some parents even decided to take another GP altogether, after having had a serious disagreement. It appeared that, once they were referred, the specialist took over the treatment, whereupon most parents stopped consulting the GP for asthma. Other relevant disciplines like nurses and physiotherapists were rarely mentioned. Parents who were satisfied with their GP mainly described their GP as supportive and expressing interest in his patients. The following are illustrations of statements concerning perceived (dis-) satisfaction with care and information provided: "We couldn't convince our GP to refer our child, finally we were referred by the locum". "I asked for an explanation and received a flood of medical jargon. They don't take you seriously." "It took a long time before someone told us that it was asthma. In the meantime you had to find out yourselves how and what to do." "We have always been well advised by our GP. $\mathrm{He}$ is always there when we need him. He listens to what we says, and he stays in contact with the pediatrician."'

\subsection{Discussion}

Considering the small and non-random group of 42 participating parents, and the overrepresentation of more highly educated respondents, the results need to be 
interpreted with some care. A possible selection bias was that dissatisfied parents, with bad experiences, might have been more motivated to participate in this study. In view of the purpose of this study, though, these biases were not expected to have a serious effect on the results of the interviews.

While the questionnaire was set up for some additional quantitative data, the focus group interviews were held to provide insight into the sort of problems parents are confronted with, their knowledge and misconceptions.

Although parents were less well informed about measures to prevent asthma symptoms, they were not expected to know all possible preventive actions because of the individual differences between children with asthma. Still some generally very useful actions were not known and some misconceptions were found. Knowledge and understanding about these actions might improve parents' ability to manage asthma adequately. The lack of confidence among parents in performing preventive actions was mainly a result of not knowing why, when and how they should perform actions. This was also indicated by several questions put to the expert present at each focus group meeting. Perceived unavailability of information from care providers might be a limiting factor affecting knowledge about preventive actions. However this finding might also indicate that the information provided lacked detail and might not have been translated into actions to be undertaken by parents in their own situations.

Although parents knew most of the actions to remedy an asthma attack they experienced similar problem as with taking preventive actions mentioned above.

Noncompliance with medication prescribed is a common problem among children with asthma (Smith, Seal \& Shaw, 1984; Spector, 1985; Klingelhofer, 1987). Several possible reasons for noncompliance were revealed. The results indicate that parents sometimes make decisions not to give medication on arbitrary grounds. Negative outcome expectations about effectiveness of medication, revealed in this study, might also strongly relate to noncompliance of patients (King, 1983; Becker \& Rosenstock, 1984). However, several times parents stiessed that their own expertise was based on their own experience with the medication, which queries the idea that noncompliance is uniformly inappropriate (Deaton, 1985). As stated by Donnelly et al. (1987) psychological resistance to the use of medications is of direct influence to noncompliance and may be amenable to education programs directed at modifying attitudes.

Furthermore, parents reported using inhaler medication to prevent or to remedy asthma symptoms. However, they did not understand the proper use of inhalers. Thus, knowing what to do and how to do it may be two different things.

The respondents appeared to be rather well informed about the causes of asthma, with the exception that they had trouble distinguishing pathogenetic mechanisms of asthma and that they failed to point out the funder the circum-

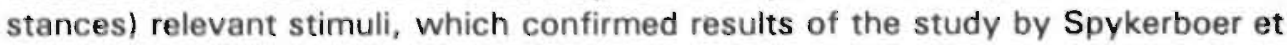
al. (1986). The virtue of the interview in this respect, thus, is that it provided information on parents ${ }^{*}$ interpretation of concepts like allergy and infections, 
which goes beyond a superficial acquaintance with the terminology. Knowing how misconceptions arise, makes them more understandable and thus facilitates an appropriate solution.

Results suggest that parental knowledge about preceding signs could be improved and on the other hand few parents held misconceptions about signs of an asthma attack.

Though the results of this study may only be generalized with ample reserve, we will list the most important recommendations for daily practice which may be implied from this study (see also Table 5).

In an education program the following aspects should receive stronger emphasis:

- The results of this study indicated the necessity of providing more extensive and systematic asthma education, especially with respect to preventive measures and (side-)effects of medicines, which might be expected to increase compliance and effectiveness of treatment;

- The use of inhalers requires better explanation; more training in skills seems to be necessary;

- Parents should be educated at an earlier stage in the disease of their child, to minimize their insecurity:

- Systematic attention to dispel misconceptions might be a worthwhile inclusion for education programs.

A combination of focus group interviews and a written questionnaire, as applied in this study, may be a useful research method, in which the two instruments together can both deliver additional data and serve as a validation of results. This was illustrated in this study, for instance, by the apparent discrepancy in results between the interviews and the questionnaire, with respect to parents' satisfaction. with the care provided by the GP. A possible explanation for the above mentioned discrepancy might be that, while a number of parents had not been seeing the GP for some time already because they were consulting a specialist, they might have been inclined to put experiences with their GP in a less negative perspective in the questionnaire. This was illustrated by some parents, who thought subsequently that the GP "couldn't help it either, because asthma is a thing for experts." The interviews, however, revealed difficulties parents had encountered with their GP in the past. The reported trend that parents stop consulting their GP for asthma symptoms was also observed in adults in a study by Ellis and Friend (1985), who found that only 3 out of 50 asthmatics kept consulting their GP for asthma symptoms.

The use of an asthma expert at the focus group meetings appeared to be helpful. The parents were more motivated to participate having the opportunity to ask questions at the end of the meeting. Further, by making a clear distinction between. the moderator, presented as a non-expert, and the asthma expert, people were prevented from asking questions to the moderator, which might have reduced the effectiveness of the meeting (Desvouges \& Smith, 1988). A final advantage of this 
task division was that it enabled the expert to pay more attention to the content. of the interview, while the moderator concentrated on the process.

This study illustrated the typical value of both focus group interviews and a structured questionnaire in assessing educational needs of a target population. The obtained findings will be used to adapt the content of existing asthma education programs to the special needs of parents off children with asthma in the 0-4 years age-group.

Table 5

Implications for practice

1. Focus group interviews are a valuable method to elicit informational needs and misconceptions of the target population

2. Both qualitative and quantitative methods of needs assessments deliver additional data and serves as a validation of results

3. Practitioners need to check systematically parental understanding of the information provided; knowing concepts does not necessarily mean that people understand them correctly

4. Practitioners should provide more oxtensive and systematic information; a standardized educational program might be helpful

5. Parents of asthmatic children should be educated at an early stage in the disease to minimize insecurity

6. Medication use (e.g. use of inhalers) requires more detailed information and actual training in skills 


\section{Development of a health education program for parents of preschool children with asthma}

Published, with some alterations, as: Mesters, I., Meertens, R., Crebolder, H., Parcel, G. (1993). Development of a health education program for parents of preschool children with asthma. Health Education Research, 8, 53-68.

\section{Abstract}

This paper presents the development and pilot testing of a self-management education program for parents of preschool children (0-4 years) with asthma, involving general practitioners, asthma nurses, community nurses and doctors of child health centers. The program intends to integrate education in the medical care provided to the child (and the parent). The program contains four manuals, one for each group of health care providers, and a bookler for parents. The manuals identify the educational tasks per discipline and regulate referral from one discipline to another. The booklet provides written information for parents.

In the development of the program, representatives from both the target population and the providers of the education were involved in needs assessment surveys. Findings of these surveys were integrated inta the design of the program. Then, a pilot study was conducted to test the efficacy of the program during group sessions. Findings indicate that the variables measured (knowledge, attitude, selfefficacy and self-management behaviors) improved significantly from pre- to posttest. Finally, the program was revised for the next phase in which the program will be evaluated in primary health care with a controlled trial. 


\subsection{Introduction}

This paper describes the process of developing a program for educating parents how to improve their management of their children's asthma (aged 0-4 years). This program or protocol tries to integrate education in the medical care provided by general practitioners, asthma nurses, community nurses and doctors of child health centers.

An educational protocoll has three functions. It is expected to identify the organization, the content and the transfer of the patient information. This means that the protocol indicates which discipline provides whal part of the information when and how.

Seven planning activities have contributed towards developing the protocol. During the first planning step, needs assessment studies among parents of asthmatic children and among the four groups of health care providers were carried out. Secondly, the information that should be conveyed to parents of asthmatic children was organized around several themes. In the third step, a pilot study was conducted to test the program in a group setting. Next, the program was revised based on the pilot study (step four). In step five the effectiveness of the program will be tested with a controlled trial. The sixth step will include the revision of the program for diffusion. And finally, we shall determine whether the nationwide diffusion of the program takes place as planned.

The purpose of this paper is to present the results of steps 1 to 4 . In the developmental process we relied heavily on the PRECEDE model (Gireen et al., 1980). However, we have tried to prevent using the terminology of that framework as much as possible, as that may complicate this paper unnecessarily for readers not familiar with the framework. The PRECEDE model is presented in Figure 1. Results of the evaluation (and revision) of the program will be described.

Asthma is a disruptive chronic disease affecting children and their farnilies in a number of ways. For instance, the social development of children might be impeded resulting in isolation (Colland, 1988). Restrictions of normal activities such as play or sleep are common (Bremberg \& Kjellman, 1985; Newacheck et al., 1986).

The majority of children with asthma experience symptoms before they reach 5 years of age (Levy \& Bell, 1984; Gerritsen, 1989a). Estimates of prevalence of asthma among children are difficult to determine due to differences in operational definition and sample selection in studies. Ellis (1983) reported a prevalence range in various populations of children in different countries from $1.37 \%$ to $11.4 \%$ or higher. Figures from Dutch primary care indicated that $4.27 \%$ of a population of 2.528 children (0-4 years) had asthma (Registratienet Huisartspraktijken, 1991 ). The incidence of asthma in preschool children is at about 8 per 1000 people (Lamberts, 1984; Van den Hoogen et al., 1985). Childhood mortality from asthma is rare. However, the $0-4$ year age group accounts for a considerable part $(20 \%)$ of the total number of hospitalizations due to asthma (Centraal Bureau voor Statistiek, $1989 \mathrm{a}, \mathrm{b})$. 
Figure 1 PRECEDE framework (adapted from Green et al., 1980) applied to asthma

EDUCATIONAL INTEAVENTION

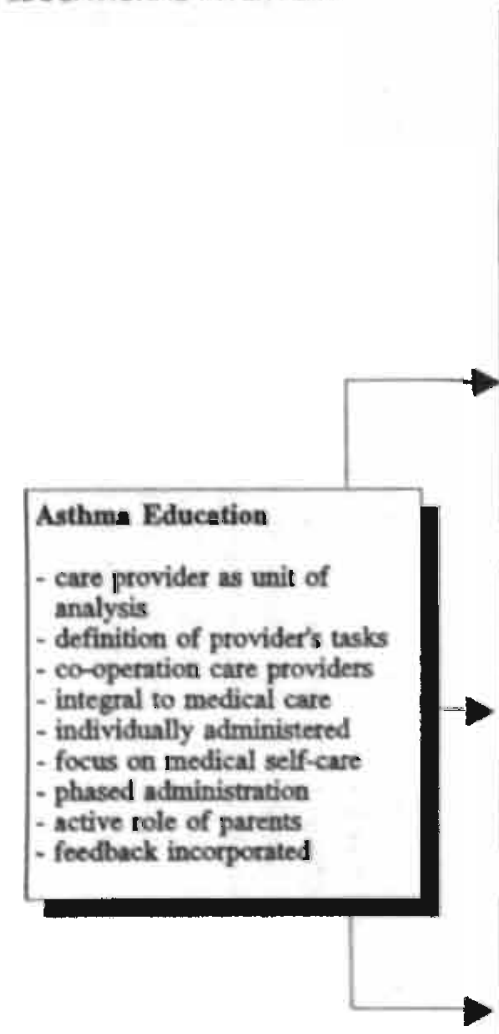

EDUCATIONAL DIAGNOSIS

\section{Predisposing Fector}

sufficient lonowledge about

asthrua:

- Jetailed information

- interconnected information

- purpose inlormation

bigh self efficacy regarding self-

management

positive attitude about asthma

care

realistic outcome expectations:

- abour self-management

- about medical care

moderate level of state-anxiety

\section{Enabling Factors}

continuity of education.

- appropriate referral

objective criteria: estimation

severity asthmn episode

skills noeded for self-

management behuviors

\section{Reinforcing Festors}

communication with care

provider:

- feedback, on management

attempt

- patient-oriented problem

$$
\text { solving }
$$

clinical management of asthma - clinical diagnosis of suthma

BEHAVIORAL DIAGNOSIS

SOCIAL AND

EPIDEMIOLOGIC

DIAGNOSIS

\section{Beherioral Facton}

Self-management behaviors:

A. Prevention of asthma attacks - predicting asthma attacks

- cotaplying with medication

- administering prescribed

medication correctly

- managing side-effeccts

* avoiding or reducing exposure to known triggens

B. Artack management:

- symptom discrimination

- complying with medication

- administering prescribed

medication correctly

- managing side-effects

- remaining calm and avoiding panic

mocituring progress of artack

C. Other behavion:

- normalizing the child's physical and social activities

- communicating effectively with health care providers

\section{Quality of lifte}

reduction of:

* social isolation

- restrictions of activities

Child's bealth status:

- visits to general practitionets

* emergency room visits

* bospital admissions. 
In the Netherlands the majority $(80 \%)$ of asthmatic patients receive medical care from their general practitioners (Voorn, 1983). In general practice asthma accounts for about $3.2 \%$ of the total number of visits a year (Centraal Bureau voor Statistiek, 1986). The nurses and pediatric doctors, working at local health centers (including child health centers), provide care for $96 \%$ of the infants and for about $84 \%$ of the 1.4 year olds (Nationale Kruisvereniging, 1988).

Thus, the literature indicates that asthma is a significant health problem among preschool children. Primary care appears to be a logical site to implement an asthma education protocol..

To a certain extent the state of health of children with asthma can be controlled by adopting particular health behavior. Several self-management programs have been developed in which education is aimed at behavioral factors (Feldman, 1987; Klingelhofer, 1987; Rachelefsky, 1987; Clark, 1989; Creer et al., 1990). These studies have enabled the identification of behaviors of school-age children with asthma (and their families) which are amenable to change and which are important to controlling asthma (Clark et al., 1980; McNabb, Wilson-Pessano \& Jacobs, 1986; Wilson-Pessano \& Mellins, 1987; Creer \& Kotses, 1989). However, no data are available yet regarding health behaviors suitable for parents of preschool-age children with asthma (Wilson-Pessano \& Mellins, 1987). Therefore, we conducted a study to elicit the needs of parents of such children. Relevant behavioral factors derived from literature and the needs assessment among parents are summarized in Figure 1.

\subsection{Needs assessment studies}

Two studies were conducted to elicit relevant behavioral determinants of managing asthma in preschool children. Educational needs of parents were assessed by conducting focus group interviews with parents of asthmatic children (0-4 years). Furthermore, the health care providers participated in a study addressing their conceptions of tasks aimed at providing asthma education. Findings of both the needs assessment studies and relevant literature have been categorized under three types of factors that may have the potential to affect health behavior. The predisposing factors are psychological factors, such as personal attitudes and knowledge. Enabling factors are important with regard to performing a relevant behavior, such as personal skills or resources. Reinforcing factors consist of the attitudes and behaviors of important referents (such as partners, family and friends) as well as physical benefits. These factors are further described below.

Predisposing factors. Included are knowledge, self-efficacy, beliefs, outcome expectations and state-anxiety. Several studies have indicated suboptimal knowledge in parents of children with asthma 'Van Asperen, Jandera, de Neef, Hill \& Law, 1986; Spykerboer et als, 1986). The focus group interviews revealed that the parents' knowledge appeared to be not only insufficient, but also mainly superficial and incoherent. Under these circumstances misconceptions can develop easily 
(Mesters, Pieterse \& Meertens, 1991). Parental knowledge seemed to be in need of improvement.

The study revealed that parents appeared to have an inadequate understanding about how to apply the information they received. Furthermore, parents' judgement of their own capability to perform self-management behaviors (self-efficacy), such as controlling an asthma attack, seemed to be insufficient (Bandura, 1986; Creer, 1987 ).

Negative beliefs about medication were revealed during the interviews, especially focusing on side-effects. Several beliefs were actually misconceptions. Furthermore, mostly all side-effects of medication were considered to be detrimental, although most of them are merely inconvenient. We concluded that this important difference should be explained in the program. Misconceptions should be addressed.

Parents ${ }^{*}$ outcome expectations in term of physical benefits for their child were low with regard to self-management behaviors such as giving medication. Noncom: pliance with medical regimen is, a common problem in children with asthma (Smith et al., 1986; Klingelhofer, 1987). Compliance might be increased by pointing out specifically any improvement in the child's condition due to medication used (Smith et al., 1986). Also providing information in the program about what effects might be expected might enhance compliance. Inadequate information and wrong interpretations often appeared to reinforce feelings of anxiety among parents. High levels of anxiety might keep parents from taking correct management decisions (Kinsman, Spector, Shucard \& Luparello, 1974; Kaptein, 1982).

Enabling factors on an individual level. Several problems experienced by parents concerned the lack of skills to manage asthma and with the lack of objective indicators of the child's physical well-being. For instance, parents indicated the need for criteria to monitor the progress of an asthma episode. This is especially important because their child is too young to express verbally how and what he or she feels. Therefore four signs of asthma trouble were included in the program (Plaut, 1988), which are objective indicators of the severity of asthma symptoms.

The focus group interviews furthermore revealed that the skills needed for the use of inhalers were lacking - a common problem among asthmatic patients (Pedersen, 1987). We concluded that skills training therefore needs to be included in the program.

Enabling factors on an organizational level. Parents and health care providers both indicated that systematic provision of asthma education was lacking. For one thing, there appears to be no continuity in care: information giving and training activities are only followed up or elaborated upon in an incidental way. This links up with the earlier mentioned suboptimal knowledge ameng parents. The protocol should try to accomplish that the education becomes an integral part of routine care of health care providers. Furthermore, referral between disciplines was perceived by parents as inadequate. Professional cooperation is essential to accomplish continuity of care. Therefore, one might conclude that the education program ought to stimulate cooperation between potential health care providers. For this purpose a survey on task conceptions was conducted. The four disciplines 
were asked to fill out a questionnaire that summarizes tasks on providing patient education to parents of asthmatic children. They could indicate what they believed were their tasks as well as their opinions of what the other disciplines" tasks might be. The underlying idea of this survey is that if care providers are expected to cooperate, they should (at least to some extent) agree with the tasks assigned to them and to others. The actual technique by which tasks were finally assigned is discussed elsewhere (Mesters, Meertens \&i Mosterd, 1991).

Reinforcing Factors. Focus group findings indicated that parents were not involved in making decisions about the management of asthma. This may result in dissatisfaction among parents which, in turn, may lead up to their performing fewer asthma management behaviors (such as giving medication). The protocol therefore should improve the interaction (communication) between patient and care provider. Furthermore, in our study parents expressed the opinion that diagnosis and treatment of asthma was delayed - a conclusion reached in other studies as well (Speight et al., 1983; Conway \& Littlewood, 1985).

If parents do not know that their child has asthma, they might not believe that following the prescribed regimen is essential. Furthermore, if asthma is not treated properly and the patient's symptoms do not improve despite medication, parents might start to doubt that their child indeed has asthma, and stop giving medication.

In summary, the adequacy of medical care (early diagnosis, appropriate treatment and mutual consent between care providers and parents about the prescribed regimen) might affect outcomes and can influence the results of a patient education program. Therefore, the medical care might need to be improved before parents can be motivated to manage asthma adequately. Figure 1 provides an overview of the needs assessment phases as suggested by the PRECEDE framework. Data and information were gathered from multiple sources, including literature and two needs assessment studies. Those items that were considered important and responsive to change are included in Figure 1.

\subsection{Method}

\subsubsection{Program development}

The educational protocol consists of five volumes: one manual for every discipline involved and a booklet for the parents. The manuals identify the specific educational tasks of a discipline and indicate when to make referrals and to whom. The manuals also focus on the transfer of information. In a special chapter, the importance of clarifying the problem, summarizing the information, checking the comprehension of the information and the necessity of limiting the number of issues per contact are all emphasized. The importance of formulating concrete aims is stressed. Emphasis is also placed on parents' involvement. in the development of a management plan by eliciting their perspectives so that desired goals will be mutually acceptable. Furthermore, it is stated that the evaluation of the actions 
performed is essential (Joos \& Hickham, 1990). To address the problem of incomplete diagnosis of asthma, a diagnostic protocol was added to the physician's manual (Van der Waart, Gill, Dekker \& Kaptein, 1987). These manuals also contained a reference to a widely available overview of medication prescribed for young children with asthma (Pauwels \& Snashall, 1986; Gerritsen, 1989b).

The booklet provides written information and can be used in a flexible way. The amount of information and the kind of information provided depends on what is most relevant and feasible for a parent at the time. The general practitioner extracts the relevant pages and gives them to parents. The booklet serves as an aide mémoire for parents. Health care providers can use the booklet to get an impression of the information they can provide to their patients.

The content of the booklet focuses on the development of knowledge about asthma and self-management skills. Social learning theory (Bandura, 1977, 1986) provided a basis for most of the intervention and learning strategies incorporated in the program. Major concepts. in social learning theory and their implications for intervention have been summarized by Perry et al. (1990). Several of these concepts mentioned by Perry et al. (1990) (behavioral capability, self-efficacy, outcome expectations) and related strategies (goal setting, skills training, selfmonitoring) were addressed or used in the program. For example, in order to prevent asthma symptoms parents learn which triggers exist and which of these triggers are relevant for their child by completing reports of every asthma episode/attack. Accordingly parents learn to evaluate the data collected on their child and to take actions to prevent or remedy symptoms. The protocol advises to learn management behaviors stepwise, under guidance of a health care provider: a step by step learning procedure should prevent parents from the feeling that "they car't do it" (c.f. self-efficacy, Bandura, 1977, 1986).

In the materials for the parents, special attention was put on explaining how different parts of the information were linked together to attain an overali understanding of asthma management: In every separate part of the text, a distinction was made between information parents should read and remember and information about what parents can do themselves (theoretical and practical information). This difference was clearly indicated with speciai headings, and symbols in the margin. The distinction was made to encourage the parents' feelings of self-control in managing asthma. Several misconceptions were addressed in the program especially to stimulate compliance with medication.

\subsubsection{Pilot study}

To justify program implementation, adequate data on the program's effectiveness and its fit with parents ${ }^{i}$ educational needs should be available. Therefore, the educational content has been tested during group sessions with parents of children with asthma. 


\subsubsection{Population}

The GPs were selected as follows. An announcement about study objectives was made in an existing regional newsletter for GPs. Subsequently, all regional GPS ( $N$ $=637$ ) were mailed information about the project, followed by telephone calls to elicit GP's interest in participation. GPs $(n=134)$ who expressed interest in joining the study were personally visited. GPs were asked to participate in either the pilot study or the clinical trial (Chapter 5). A total of 45 GPs indicated willingness to enrol patients for the pilot study. Parents, who were approached by their GP, could enrol by returning a form to the research team. The parents were eligible to participate if their children met the following criteria: asthma should be diagnosed by the general practitioners, aged $0-4$ years and recently diagnosed (preferably within the past 6 months). GPs had to enrol patients within three months time. Fifty parents (respondents and partners), distributed over six groups, attended the sessions. The parents were enrolled by $17 \mathrm{GPs}$. All participants were present at all three group sessions. The sessions were led by physicians or the researcher.

Table 1

Sociodemographic characteristics of the respondenis $(N=31)$

Variables

\begin{tabular}{|c|c|c|c|c|}
\hline Parental age (years) & & $\begin{array}{r}M \\
30.5\end{array}$ & $\begin{array}{l}S D \\
2.9\end{array}$ & $\begin{array}{l}\text { Range } \\
25-36\end{array}$ \\
\hline Child's age (years) & & 2.6 & 1.3 & $0.6-5$ \\
\hline Symptoms since (months) & & 19.8 & 14.2 & $2-53$ \\
\hline Treatment since (months) & & 12.0 & 10.6 & $1-36$ \\
\hline Diagnosis know since (months) & & 9.0 & 7.3 & $1-36$ \\
\hline Parental education $\left(X_{1}\right)$ & & $\begin{array}{l}\text { Low } \\
22.6\end{array}$ & $\begin{array}{r}\text { Middle } \\
54.8\end{array}$ & $\begin{array}{l}\text { High } \\
22.6\end{array}$ \\
\hline Trouble with management $(x)$ & $\begin{array}{c}\text { None } \\
22.6\end{array}$ & $\begin{array}{r}\text { Some } \\
58.1\end{array}$ & $\begin{array}{r}\text { Much } \\
9.7\end{array}$ & $\begin{array}{r}\text { Don't } \\
\text { know } \\
9.7\end{array}$ \\
\hline & & & $\begin{array}{r}\text { Yes } \\
67.7\end{array}$ & $\begin{array}{r}\text { No } \\
32.3\end{array}$ \\
\hline \multicolumn{5}{|l|}{ Having asthma medicines $(\%)$} \\
\hline $\begin{array}{l}\text { Sex child }(\%) \\
\text { Sex parent }(\%)\end{array}$ & & & $\begin{array}{l}\text { Male } \\
64.5 \\
16.1\end{array}$ & $\begin{array}{r}\text { Female } \\
35.5 \\
83.9\end{array}$ \\
\hline
\end{tabular}

The sociodemographic characteristics of the respondents and the children in the study are summarized in Table 1. Most children were represented in the analysis by their mothers $(n=26)$. The children had a diagnosis of asthma for a longer time (on average) than the target period of one half year. As might be expected in 
this young age group, more boys than girls were involved in the study (Schachter, Doyle \& Beck, 1984; Dodge, Cline \& Burrows, 1986; Evans, Mullally et al., 19871. One might assume that general practitioners treat mild to moderate asthma. Patients with severe asthma are more likely to be treated by specialists. Most children involved in this study seem to have mild or moderate asthma symptoms; most parents indicated that they had little trouble managing asthma. Only twothirds of the patients have asthma medicines. Results indicate the children had been treated for asthma symptoms for three months on average before parents were told that their child had asthma.

\subsubsection{Instrument development}

The questionnaire (see Appendix 3) was pretested for readability and uniform interpretation with 10 mothers of young children with asthma. For every subscale in the questionnaire the Cronbach's alpha $(a)$, a measure of internal consistency, was assessed and will be reported with every scale. The questionnaire was constructed to inquire about the following factors:

1) Knowledge. The knowledge questionnaire was partly derived from the adult knowledge instrument developed by Parcel et al. (1980). The instrument used was constructed in a 'true-false' format, testing for an understanding of basic concepts about the nature of asthma and general management procedures. The questionnaire consisted of 73 items $(\sigma=.81)$.

2) Attitude. To assess the attitude of the parents, a 24 -item survey $(a=59)$ was developed. This survey was partly based on the adult attitude survey used in Living with Asthma (Creer et al., 1986). The questions were measured with a five-point bipolar rating scale (range: $+2 /-2$ ).

3) Self-efficacy. The 32 questions about the perceived behavioral capability of parents $(a=.93)$ were measured with a six-point bipolar rating scale, including a 'not applicable' category. Three subscales can be distinguished: prevention, treatment and monitoring of asthma symptoms.

4) Management behaviors. Three management categories were constructed to inquire in detail about the extent to which self-management actions were performed: preventive actions, actions to treat an attack and actions to monitor an asthma attack or episode. The questions were measured on a five-point scale 10 indicating that a certain behavior is never performed and 4 indicating that the behavior is always performed). If necessary, people couid use the option 'not applicable' ( $a=.92)$.

5) State-anxiety. People who experience extremely high and low levels of anxiety during asthma attacks (state-anxiety) are less likely to take appropriate management decisions (Kinsman et al., 1974; Kaptein, 1982). Because of the possible influence of differences in level of state-anxiety among parents on parental management of asthma, we concluded that state-anxiety should be measured in the evaluation of the program. For this purpose the Dutch version of the 'state-anxiety inventory' was used (Van der Ploeg et al., 1980). This inventory was reduced from 20 to 8 items, containing four anxiety present 
items (tense, nervous, indecisive and worried) and four anxiety absent items (calm, at ease, certain and relaxed). When completing this scale, parents were instructed to describe how they felt during one of their child's asthma attack. or asthma episode. The items were scored on a four-point scale in which the higher the score indicated the higher the state-anxiety $(a=.91)$.

\subsubsection{Data collection}

Data were collected by means of a written questionnaire. The pretest questionnaire was sent (1 week before the first meeting) to the participant's home and was collected at the start of the first meeting. Three weeks later the posttest questionnaire was handed out at the end of the last (third) meeting. The respondents could return the questionnaire in a postage-paid envelope. Four questionnaires were excluded from analysis because either the parent did return the baseline questionnaire too late $(n=1)$ or the parent had not returned the posttest questionnaire $(n=3)$, reducing the total sample from 35 to 31 respondents.

\subsubsection{Data analysis}

Student's t-tests for paired comparisons were used to test for significant differences $(\rho<.05)$ from before to after the educational program.

The mean scores for the questions on asthma management. behaviors and selfefficacy were corrected for questions which were not applicable. Comparisons measures for knowledge questions were constructed and based on the number of correct answers. People with more than one missing value on the state-anixiety scale were not included in the analysis $(n=2)$. Furthermore, frequencies were calculated for the sociodemographic items in the questionnaire. Finally, correlations were performed between the sociodemographic variables and the gainscores (preto post-intervention scores).

\subsection{Results}

Parents increased significantly in knowledge (see Table 2). The comparative responses on the pre- and posttest to individual items on the knowiedge instrument were analyzed. In particular, knowledge improved about measures that can be taken to prevent an attack, about what happens in the lungs during an attack, about causes of an attack and estimation of the severity of an attack.

Parents furthermore acquired a more positive attitude to asthma (see Table 2). Their attitude became more positive towards giving asthma medication to prevent and/or to treat asthrna symptoms and towards taking actions to prevent asthma symptoms in general.

Parents' self-efficacy increased significantly indicating that after the program parents had more confidence in performing self-management behaviors (see Table 
2). A significant $(p<.05)$ increase was also found in each separate subscale (prevention, monitoring and treatment).

On the posttest questions parents reported more self-management behaviors and reported an increase in the frequencies at which they performed certain actions (see Table 2). Significant $(p<.05)$ increases were found for each of the preventive, monitoring and treatment behavior scales. Table 2 further shows that the parents' level of state-anxiety did not change after participation in the study.

Correlations were performed between parents' gainscores and the demographic variables, mentioned in Table 1. A significant correlation was obtained only between parents' level of education and their gain in knowledge $r_{n}=-45$; $p<.01)$, indicating that the higher the parent's level of education, the less they profited from the education they received.

Table 2

Mean scores before and after program $(N=31)$

\begin{tabular}{|c|c|c|c|c|c|c|}
\hline \multirow[b]{2}{*}{ Scales } & \multicolumn{2}{|c|}{ Pretest } & \multicolumn{2}{|c|}{ Posttest } & \multirow[b]{2}{*}{$\begin{array}{c}t * \\
\text { value }\end{array}$} & \multirow[b]{2}{*}{$p<$} \\
\hline & $M$ & $S D$ & $M$ & $S D$ & & \\
\hline Knowledge & 38.64 & 11.87 & 58.96 & 7.81 & 9.24 & .001 \\
\hline Attitude & 26.12 & 6.53 & 35.83 & 5.23 & -8.43 & .001 \\
\hline Self-efficacy & .28 & .47 & .68 & .54 & -5.30 & .001 \\
\hline Self-management behaviors & 2.42 & .52 & 2.84 & 68 & -4.00 & .001 \\
\hline State-anxiety ${ }^{\bullet}$ & 22.17 & 3.97 & 21.48 & 4.95 & 1.07 & .30 \\
\hline
\end{tabular}

Note. of $=30 ; \cdot N=29 ; d f=28$

\subsection{Discussion}

Preliminary findings indicate that the results of the protocol are promising. Parents have, after they have been confronted with the program, more (practical) knowledge on asthma and a more positive attitude towards asthma than before. Furthermore, they feel more confident that they can perform the behaviors necessary to manage their child's asthma, and report an increase in the number of behaviors performed and that they more frequently perform such behaviors. Despite these promising findings, some critical remarks can be made. First, although parents improved significantly on the self-management behavior measure, it is not likely that parents had had the opportunity yet to perform these behaviors. Thus, this finding is best interpreted as parents' intention to perform a behavior or to perform a behavior more frequently. Second, parents' level of state-anxiety was high and comparable to the level of state-anxiety in adult asthmatics (outpatients) in a Dutch study by Kaptein (1982). One might expect that when parents become more able to manage asthma attacks, they will become less anxious when an 
attack occurs. In our study, however, no significant reduction in level of anxiety was obtained. Perhaps the duration of the study was too short to detect a change in a psychological factor like state-anxiety. However, parents" level of state-anxiety might not be expected to decrease very much: even if parents are better able to manage asthma attacks, their child's asthma attack will always make them feel worried and tense. Last, but not least, the pilot study is limited because of the small sample and the lack of a control group. Nevertheless, parents in the study came from different medical practices in several cities in the Netherlands. Furthermore, the study was carried out in a short period of time. This makes it unlikely that history or maturation influenced the changes we observed.

An interesting finding from the pilot study is the discrepancy between the time the treatment for asthma symptoms began and the time the child was diagnosed (Table 1). The meetings with the parents revealed several explanations for this difference. Parents' reports indicated that the principle "if asthma medication works, then it is asthma" might be commonly used in asthma care for young children. Another explanation might be that the physicians postponed revealing the diagnosis because they believed that the parents might be shocked by hearing that their child has asthma. Another explanation for this discrepancy might be that some physicians treated the symptoms with other medication (e.g. antibiotics) than those for asthma and after some time realized that they were dealing with asthma. Again the discrepancy between time of treatment and time of diagnosis illustrates the need for an education program which includes a diagnostic protocol and an overview of appropriate medication for young children with asthma in order to provide adequate advice for parents in the management of their child's asthma.

Significant negative correlations between level of education and knowledge gain suggest that parents with a lower level of education are more likely to benefit most from the program. An explanation of this finding might be that parents with a higher education already had better scores, and thus could show less improvement on knowledge and attitude than parents with a lower level of education.

The future course of the project will show to what extent the protocol can achieve the results described in an ambulatory medical care setting.

\subsection{Revision of the program}

Some minor revisions in the content of the protocol (merely textual) were made as a result of the pilot study. However, a more important revision dealt with the transfer of the information. During the sessions it appeared that checking parental understanding of the information provided is very important. During the group session there was enough time to provide information, to repeat and to check the understanding of information provided. However, this approach might be too time consuming in a physician's office. Besides checking understanding can be easily forgotten in a busy practice. Therefore, a check on understanding was built into the educational program: parents will receive the information (on paper) prior to the 
contact with the health care provider, and read the information at home. The information will be discussed by one of the health care providers during a next contact. For instance, the general practitioner hands out the pages on adapting homes. This information will be discussed by the community nurses during a home visit. Because parents will have studied the information, health care providers can focus on rehearsing and summarizing important information, checking the comprehension of the information and eliciting questions from the parents. Again, more attention can be paid to tailoring the asthma information to the specific situation of the patient. This approach is expected to stimulate parental involvement in the care provider-parent interaction, which is considered to enhance adherence (Bruhn, 1983). After the contact the written information serves as an aide mémoire for parents.

\subsection{Implications for practice}

Perhaps the most important implication for practice is that now an education program is available for preschool children with asthma, which seems to have a positive effect on parents' (practical) asthma knowledge, attitude towards asthma, self-efficacy perceptions and self-management behaviors. Furthermore, we feel that the systematic way in which the program was developed, strongly contributed. to the detected positive effects. The steps that seem especially important in the process of developing an educational protocol meant to be used by several disciplines will be given now, formulated as suggestions or advise.

- Tailor the program to the needs, existing knowledge and misconceptions of the target population.

- Elicit task conceptions and specific task-related knowledge of relevant health care providers, and get at their idea on what tasks other disciplines should perform.

- A preliminary evaluation of the program in a group setting may give valuable suggestions on how the program can be improved.

Furthermore, we feel that:

- involvement of the general practitioner is necessary to provide patient specific information about asthma. Physicians have valuable information, for instance, about severity, individual triggers or medication treatment. This information is needed to tailor the information to the individual patients. Such information can be provided by parents themselves, but needs to be checked with the general practitioner who treats the patient.

- educational principles, such as checking comprehension of information, should be incorporated in the procedure in which the protocol is to be used. This might help health care providers to apply such skills in their patient education. 



\section{The effectiveness of a multidisciplinary education protocol for children with asthma (0-4 years) in primary health care}

Submitted in a shortened version as: Mesters, I., Meertens, R., Kok, G. \& Parcel, $G$. The effectiveness of a multidisciplinary education protocol for children with asthma (0-4 years) in primary health care.

\section{Abstract}

This report presents the short term and long term evaluation results of an education protocol developed for parents of children with asthma (0-4 years). The program is integrated into primary health care and involves general practitioners, community nurses, asthma nurses and doctors in child health care centers. The post test results of a randomized controlled study show that parents participating in the program have significantly more knowledge, a more favorable attitude and a higher selfefficacy score. Furthermore, they report performing self-management behaviors more frequently than parents in the control group. Long term results were collected from parents in the experimental group only. The waiting list control group could no longer be used as controls because they had received the intervention materials after the posttest. Data showed that changes measured directly after the intervention period of six months were sustained at one-year follow-up. Eventually state-anxiety, which had not shown an important change at the posttest, appeared to have decreased significantly at follow-up. Furthermore, medical care utilization data were collected from physician's records on the experimental group and a new control group which was used only for collecting medical care utilization data and data on the severity of patients' asthma. Results indicated that the experimental group showed a significantly greater decrease than the control group in the number of contacts with the GiP, the number of emergency visits to the physician's office and the number of hospital admissions. The two indicators of asthma severity as 
reported by the GP demonstrated that severity of symptoms decreased more in the experimental group than in the control group.

Both the short term and long term process evaluation indicated that cooperation between the disciplines involved took. place as described in the protocol, though some deviations were observed. Furthermore, data were collected on the extent. and the way the intervention materials were used by the disciplines (and parents) involved. Results showed that the program was implemented and maintained satisfactorily with respect to both the extent to which the protocol modules were distributed and the way the information was handled by the health care providers. Health care providers who had worked with the protocol stated that communication with the parents had improved. The disciplines indicated their satisfaction with the materials and signalled their intentions to continue to use the program. Parents evaluated the program positively on both occasions.

\subsection{Introduction}

For childhood asthma, patient education has been demonstrated to be effective at increasing the ability of patients (and their parents) to manage the disease (Wigal et al., 1990). Epidemiological studies have shown that for the majority of the children the onset of asthma starts before the age of three (Gerritsen, 1989a). Nevertheless, literature revealed no asthma education programs were targeted specifically at preschool children. To address this lack, we developed and evaluated an asthma education program for such a target group.

Obviously, successful educational programs will only have a significant impact If they reach a considerable proportion of the target population (Parcelet al., 1989). In the Netherlands $80 \%$ of the people with asthma are treated in primary care (Voorn, 1983; Schipper, 1988). With this in mind, the education protocol was designed for use by four primary care disciplines considered relevant for preschool children: GPs, community nurses, asthma nurses and doctors in child health centers. Education efforts by primary health care providers are usually not integrated into their routine care, and education is often uncoordinated and unsystematic. Literature suggests that education for patients may be optimized by educational protocols that guide the provision of systematic and planned patient education as well as the cooperation between relevant disciplines (Kanters \& Jonkers, 1988).

To establish a firm basis for the protocol, several studies were carried out (Mesters, Meertens et al., 1991; Mesters, Pieterse et al., 1991; Mesters, Meertens. Crebolder \& Parcel, 1993). First, a study was conducted to divide educational tasks among the disciplines involved in such a way that cooperation was based on their mutual consensus on task conceptions (Mesters, Meertens et al., 1991). A representative sample of each discipline completed a questionnaire to indicate their conceptions about each discipline's tasks, including their own. Furthermore, task specific knowledge questions were added to the questionnaire. The data from the survey were used to assign tasks in the program to the different groups of health 
care providers, on basis of the principle that task assignment should be linked as far as possible with existing task conceptions and the existing level of task specific knowledge (Mesters, Meertens et al., 1991). Finally, it was concluded that the protocol should enable health care providers to improve their asthma knowledge in order to provide appropriate education. The next study provided an educationall needs assessment for parents of 0-4 year-old children with asthma because no specific information on their level of knowledge, needs, beliefs and misconceptions about asthma was available (Mesters, Pieterse et al., 1991). Such findings were needed to tailor the content of the educational protocol as closely as possible to the needs of the target group. The study's results indicated that parents had a need for practical knowledge about asthma. Important knowledge deficits and misconceptions, which might contribute to noncompliance with self-management behavior, were revealed and subsequently addressed in the protocol.

The results of the first two studies were used to design the intervention which consisted of a manual with guidelines for each discipline to follow: a) to deliver a series of educational modules for the parents to review with the health care provider and to use at home; and b) to decide when to refer patients to other disciplines for discussion of information. The parent-booklet can be used in a flexible way. The number and kind of modules provided depended on what the GP considered most relevant for a parent at the time. Relevant modules could be selected by extracting relevant pages from the parent-booklet. At home parents decided, after reading the information, what topics they wanted to discuss with their care provider during a subsequent contact. During this contact the health care provider could focus on rehearsing and summarizing important information, checking comprehension of the information, eliciting questions from parents and tailoring the information to parents' specific situation and interest. Every asthma information topic in the parent-booklet was rephrased at the end in action terms to ensure that parents knew what actions they could take (Barthelomew et al., 1991).

Prior to its use in primary care, the program was pilot tested during three group sessions. Results showed a significant favorable change in knowledge, attitude, self-efficacy and report of asthma self-management behaviors of parents over a three-week period (Mesters et al., 1993).

The purpose of the study described in this article is:

1) to evaluate the effect of the asthma education program, which is integrated in primary care, on parent's knowledge, attitudes, self-efficacy self-management behavior and state-anxiety by a randomized trial;

2) to determine to what extent health care providers implement the protocol and how they cooperate with each other; and to gather information on how care givers and parents evaluate the protocol;

After the research questions 1 and 2 had been studied, the control group received. the intervention materials. Therefore, they could no longer be used as controls for the third and fourth research questions, which had the following purpose:

3) to evaluate to what extent effects addressed in questions 1 and 2 were sustained in the experimental group at one year follow-up; 
4) to evaluate the asthma program's impact on medical care utilization variables (such as number of visits to physician's offices) and severity of asthma symptoms) at one year follow-up. For this purpose a new control group was used.

\subsection{Methods}

\subsubsection{Recruitment and randomization}

The GPs were selected as follows. An announcement about study objectives was made in an existing regional newsletter for GPS. Subsequently, all regional GPS ( $N$ $=637$ ) were mailed information about the project, followed by telephone calls to elicit GP's interest in participation. We personally visited GPs $(n=134$ ) who expressed interest in joining the study. Finally, of the 89 GPs who were willing to participate in the clinica! trial, 67 GPs succeeded, within the fixed time of six months, in enrolling patients who met the criteria for inclusion in the study. The criteria vere: (1) child not older than four years; (2) diagnosis of asthma confirmed by the GP; and (3) child recently diagnosed (0-6 months ago). Parents, who were approached by their GP, could enroll by returning a form to the research team. On this form parents $(N=108$ ) indicated the (local) child health center they were visiting. The parents received a letter from the research team in which the research design (experimental and control group) was explained, and in which parents were told that drawing lots would decide which GPs would be in the control or the experimental group. Next, the asthma nurses $(N=12)$, the community nurses ( $N$ $=40)$ and the doctors $(N=26)$ working at these centers were recruited to participate in the study to provide care and education to our treatment group. Only two health care providers of the centers declined to participate because they were to retire within a few months.

A pretest-posttest waiting list control group design was used with random assignment of general practices to treatment and control group for studying research question 1. Before randomization, practices were stratified on three characteristics: type of practice (solo, paired or group practice), number of enrolled GPs per practice and number of enrolled patients per GP. Assignment of a general practice to a particular condition automatically implied assignment of all related subjects to this condition (GPs, other relevant care providers and patients). Table 1 provides an overview of GPs and patients per type of practice, and is relevant for all research questions but research question 4.

Next, the educational materials and instruction guidelines were mailed to the health care providers two weeks before a 10-15 minute personal visit by a member of the research team, who rehearsed the guidelines, checked understanding of the guidelines and addressed misconceptions by the care providers. The following introduction strategies, based on diffusion theory, were used during the introduction of the program in the experimental group. Rogers (1983) identifies several features of an innovation that will influence the speed and the degree in which it will reach 
its target population: compatibility, relative advantage, complexity, trialability and observability. Respectively, the higher the compatibility of the product with values, needs or norms of the target population, the higher the perceived relative advantage of the protocol and the easier it is to use the protocol, the more likely it is that the protocol will be implemeted. New ideas that can be tried on a limited basis or scale are more likely to be adopted. And finally, the more visible the positive results are to others, the more communication about the product is stimulated, which will promote greater adoption and diffusion of an innovation. It follows that the message, delivered to the care providers by word of mouth and in writing, referred to the participation of people of the same profession in the development of the protocol; the advantages, like favorable behavioral effects, more satisfied patients, automatic administration of the information provided, were stressed. The uncomplicated use of the protocol was demonstrated; care providers could start using the protocol with only one patient. The care providers were also told to look for positive signs such as better self-management by parents.

For research question 2 a one group posttest design was applied. For research question 3 a one-group pre-/ posttest design was used, and for research question 4 a non-equivalent control group design was adopted (Campbell \& Stanley, 1966). The new control group of patients, who were studied with respect to research question 4 on medical consumption and severity of asthma, were selected from a computerized data base of a registration network of family practices in the Netherlands (Metsemakers et al., 1992). Because of the need for comparability with the experimental group, the patients were selected on the basis of the same criteria as mentioned earlier. It was decided to select a maximum of three patients per GP", resulting in 19 GPs and 42 patients. The general practices were located in the same geographical areal as the practices in the experimental group.

Table 1

Number of GPs and patients in treatment and control group per type of practice

General practitioners

Patients

\begin{tabular}{lcccc}
\hline & $\begin{array}{c}\text { Experimental } \\
\text { group }\end{array}$ & Control group & $\begin{array}{c}\text { Experimental } \\
\text { group }\end{array}$ & Control group \\
\hline Solo & 13 & 12 & 19 & 19 \\
Duo & 13 & 14 & 25 & 22 \\
Group & 9 & 6 & 13 & 10 \\
\hline
\end{tabular}




\subsubsection{Data collection procedures}

For the purpose of evaluating the effect of the program on the educational and behavioral variables, data from parents were collected by written questionnaires which were sent to parents" homes before and after the intervention period, and at one year follow-up. The questionnaires could be returned to the research team in a pre-stamped envelope. Parents in both the treatment and control group received the pretest questionnaire in March 1990. No other contact with the research team occurred during the intervention period. After six months, all parents in both conditions were reassessed in an identical manner. The posttest questionnaire included some process evaluation questions. The questionnaire was administered again at one year follow-up in the experimental group only.

For the posttest, a 15-minute telephone survey was conducted with the health care providers to inquire about program use. To minimize socially desirable answers from the care providers, these structured telephone interviews were conducted by several freelancers. Subsequently, those care providers who had actually implemented the program were visited personally by members of the project to obtain additional (qualitative) data. During these visits to the GPs, program use was also directly measured by documenting which modules were missing from the parent booklet. A missing module normally indicated that the GP gave the module to the parents. To avoid any distortion of findings, physicians were not informed in advance about this method of data collection. For the one year follow-up, a written survey (accompanied with a pre-stamped envelope) which was similar to the 15 . minute posttest interview, was mailed to the health care providers. The reason for this change in procedure was to prevent causing extra inconvenience by settling a date and time which the disciplines were expected to keep. Now, it was left up to the disciplines themselves to decide when to fill out the questionnaire.

Medical care utilization data and data on severity of asthma symptoms were collected with a written questionnaire. These medical findings, originating from medical records, were provided by the GPs. Data were collected retrospectively on the year preceding the pretest (01-03-'89 till 01-03-'90) and on the period of one year after the posttest (01-09.'90 till 01-09-'91). GPs were informed about our intention to collect this data after the data collection periods had passed.

\subsubsection{Measurement instruments}

The questionnaires measuring outcomes at the parent's level with respect to knowledge ( 73 questions), attitude ( 24 questions), self-efficacy ( 32 questions), selfmanagement behaviors ( 20 questions) and state-anxiety ( 8 questions) can be obtained by contacting the first author. Cronbach's alphas for all scales were above .80 , except for the attitude scale $(a=.33)$. The latter alpha may be low because the attitude items addressed quite different issues. Examples of these issues are: "it is important to take asthma medicines on time" and "People with asthma can 
be successful". Therefore, we will also indicate in the results for single attitude items the result section. Questions on knowledge dealt with the etiology, pathogenesis, pathophysiology, symptomatology, precipitants and outcomes of asthma, asthma medication and the rationale for self-management actions. The knowledge questions were measured in a "true-false-l don't know" format. The parents' attitude measure included statements about taking preventive actions, treating asthma attacks and monitoring asthma attacks. Also, more general statements on asthma were included. The questions were measured with a five point bipolar rating scale (range $+2 /-2$ ). With respect to self-efficacy and self-management behaviors, three main categories were distinguished: prevention, monitoring and treatment of attacks. The prevention scale items dealt with the prediction of asthma attacks, preparations to avoid attacks and the use of preventive medication as prescribed. The treatment scale consisted of items dealing with treating an attack by symptom detection and the use of medication. The monitoring scale included items on estimating severity and progress of attack. The self-efficacy questions were measured with a six-point bipolar rating scale (very well-very poorly), including a "not applicable" category. For example: "Imagine giving your child medicines: how well can you determine the dosage?" The questions on self-management behavior measured relative performance frequency on a five point scale ( 0 indicating that a certain behavior is never performed and 4 indicating that it is always performed). If necessary, parents could use the option "not applicable". For exampie: "To what extent do you give your child medication to stop an asthma attack?". State-anxiety questions were derived from the Dutch version of the Spielberger State-Trait Anxiety Inventory (Van der Pioeg et al., 1980). Oniy those items were included that described reasonable feelings parents experience during their child's asthma attack. The selection was made by parents who were involved in the pretest of the questionnaire, which has been described in Chapter 4. Our inventory contained four anxiety present items (tense, nervous, indecisive and worried) and four anxiety absent items (calm, at ease, certain and ralaxed). Level of state-anxiety was measured in this study to check whether parents would become more anxious during asthma attacks when health care providers, by providing education, had given them more responsibility for handling their child's asthma attacks.

The telephone survey and the written questionnaire among health care providers inquired about program use, care provider's satisfaction with the workability of the protocol and program re-invention. Re-invention is defined by Rogers (1983) as the degree to which an innovation is changed or modified by a user in the process of its adoption and implementation.

Medical care utilization was measured by the number of emergency contacts and non-emergency contacts the GPs had with the patients because of asthma. The number of telephone contacts were registered. Referral of patients to specialists by the GP, emergency visits of patients to the hospital, referral to the disciplines for home care and other referrals were registered. Furthermore, the number of hospital admissions, if applicable, were recorded. Indicators of severity of asthma symptoms were assessed by the physician's impression of the duration of asthma 
episodes and the GPS' perception of asthma severity as measured on a six-point category scale, ranging from no symptoms to severe symptoms (National Heart Lung and Blood Institute \& National Heart and Lung Institute, 1992)

\subsubsection{Analysis procedures}

Cronbach's alpha (a) was used to measure the internal consistency of the scales in the questionnaire. Chi-square tests were used to examine differences in proportions of the categorical variables. Strength of relationship between variables was determined with the Spearman rank $\left(r_{\mathrm{s}}\right)$ or the Pearson product moment correlation coefficient $(r)$. Multiple linear regression was used to determine the degree of association between the educational variables (knowledge, attitude, selfefficacy) with self-management behavior (pretest data). Furthermore, regression was used in the randomized trial to predict gain in self-management behaviors from gain in the educational variables. To assess the effects of the protocol, multivariate repeated measures analysis of variance was performed on the five dependent variables: attitude, knowledge, self-efficacy. self-management behavior and stateanxiety. The independent variables were "condition" (treatment and control group) and "time" (pretest and posttest). Overall analyses in the randomized trial were peiformed with GPs as unit of analysis (there were only three practices which had more than one practitioner involved in the study). Only when indicated, analyses were carried out on patient level. Pairwise t-tests were applied to test differences in means of the behavioral variables measured among the experimental group at posttest and one year follow-up. Analysis of covariance was employed to determine whether medical care utilization and asthma severity at posttest were different in the experimental and the control group, after holding constant prior scores on the variables mentioned.

\subsection{Results}

\subsubsection{Sample characteristics}

The total sample for the randomized trial consisted of 67 GPs, with 32 in the control and 35 in the treatment group. Four GPs in the treatment group had patients with missing data on the posttest, which reduced the sample from 35 to 31 . Chisquare statistics revealed no significant difference between the study groups with respect to child's gender, respondent's gender, parental education and the degree to which parents perceive asthma as a problem. The sex ratio for the 108 children was 2 boys $(N=70): 1.08$ girls $(N=38)$. The majority of the respondents were women $(n=91 ; 85 \%)$. Thirty-three percent $(n=35)$ of the parents reported they had had lower (vocational) education; $54.7 \%(n=58)$ had had an intermediate 
education (lower and higher general secondary education) and $12.3 \%(n=13)$ had obtained at least a pre-university, university or higher vocational education. Two parents did not indicate their educational background. About one quarter in $=23 ; 24.2 \%$ ) of the respondents indicated that managing asthma symptoms gave them no problems, $48.1 \%(n=52)$ experienced some problems, $18.9 \%(n=20)$ of the parents experienced a great number of problems and $12 \%(n=13)$ indicated that they did not know.

Multivariate analysis of the sociodemographic data determined that the multivariate $F(F(5,58)=2.59 ; p<.04)$ was significantly different between the study groups. Further analysis of the univariate findings indicated that respondents in the control group reported a significantly $(p<.05)$ longer period since treatment $(M=12.83$ months; $S D=7.82)$ and diagnosis $(M=12.36$ months: $S D=8.13)$ than the respondents in the treatment group ("time since treatment." $M=7.88$ months, $S D=7.17$ and "time since diagnosis" $M=7.45$ months, $S D=6.26$ ). Whether these differences will be of any importance to the study results will be reported later. No univariate significant differences between the two study' groups were revealed in parental age, child's age and time since first experience of symptoms. The children's age rangeci from 3 months to 5 years and 2 months $(M$ $=2.44$ years, $S D=1.04)$. The mean age of the parents was 30.76 years $(S D=$ 2.99). On average children experienced the first symptoms 13.88 months ago (SD $=9.121$.

In the nonequivalent study, $28 \mathrm{GPs}$ of the experimental group provided data on 46 patients. Some loss of data occurred because patients $(n=5)$ changed GiPs and took their medical record with them. The control group finally consisted of 38 patients from $18 \mathrm{GPS}$. Two patients selected initially were not used for analysis because the GPs suspected asthma, but had not yet confirmed the diagnosis. In addition, two patients had incomplete records because they' transferred out of the practice in the middle of one of the research periods and were eliminated from analysis as well. Children's age in the experimental group was comparable to the age of the children in the control group, univariate $F(1,52)=1.24, p<.27$. Furthermore, no differences were revealed with respect to child's gender, $\chi^{2}$ (1, $N=53)=1.61, \rho<.21$, and asthma severity as estimated by the GP, $\chi^{2}(1, N$

$=46)=.01, \rho<.98$. Most children had mild $(35 \%)$ to moderate $(40 \%)$ asthma (Appendix 5).

\subsubsection{Process evaluation}

\subsubsection{Transfer of education}

All GPs participated in the telephone interview. Except for one, all GPs reported having given written information to their patients. The protocol is baseci on the principle that providing extensive information outright should be avoided and 
checking comprehension of the information provided should be included in the GPs' routine. Therefore, we asked GPs whether they provided the written information in parts (per module) and whether they discussed the information during the next contact. During the posttest, most modules were distributed among parents by the GPs to a satisfactory degree; the least distributed module was handed out to $54 \%$ of the parents. Only five GPs did not spread the distribution of the information over several contacts, but gave out the complete booklet to parents at one visit. Three of these care providers had no further contact with their patients and the other two GPs determined with the parents in what order and frequency they were going to ciscuss the information. The majority of the GPs indicated that they discussed all or nearly all the information they provided. Although some of them gave some information when handing out the written materials, most GPs discussed the information after each parent had read the materials at home. On average, five contacts with parents turned out to be sufficient to discuss all the information that a GP considered relevant for the parent in question. The average interval between the contacts was slightly less than three weeks. Nearly all the GPs expressed their positive intention to use the protocol for other patients. At one year follow-up 31 GPs participated in the survey. About 22 physicians still used the protocol. The protocol was continued with 18 patients of the intervention study and was started with 4.8 new patients. All GPs, except for one, positively intended to continue using the protocol. Reasons for not using the protocol in the year after the posttest were "no new asthma patient seen" and "forgotten to use it". All users utilized the parent booklet. Several patients $(n=36)$ received the entire booklet during a single contact. The remaining 12 patients received the information per module in an average of four contacts. The information had been discussed with the majority of the "new" parents $(n=42)$.

Only 32 of the community nurses involved in the treatment group of the project $(N=40)$ could be reached for a posttest interview. Twelve of the community nurses interviewed indicated that they had used the protocol, although not always for a patient who was involved in our study. Most $(n=27)$ community nurses expressed their positive intention to use or to keep using the education protocol. Four nurses did not know yet and one nurse admitted having no intention of using the protocol. At one year follow-up 27 community nurses participated in the survey. Of these nurses eight had used the protocol with 3 patients on average. Others did not use the protocol because they had not had contact with relevant asthma patients $(n=10)$, no parents were encountered who had a parent-booklet $(n=$ 13), or because they had forgotten and/or no intention of using it $(n=1)$. The nurses interviewed positively intended to continue using the protocol.

Of the 26 doctors in the child health care center, 15 could be reached for an posttest interview. None of the interviewees had used the protocol. Still, they did intend to use the protocol in future. The follow-up survey, returned by 10 doctors, revealed that two had used the protocol, although all doctors had had contact with asthmatic children. The two users were the only ones whose parents were in the 
possession of the parent-booklet. Reason for non-response or non-use was that the doctors had not had contact with parents having asthma-booklets.

In all, 12 asthma nurses were involved in the project. Four nurses were lost for follow-up because they changed jobs. Of the remaining eight nurses, three had provided care for parents in the study. Two asthma nurses used the protocol during their contact with a parent. One nurse discussed information with a parent, the other stated that the parents already understood the information presented. Most interviewees said they intended to continue or to start using the protocol. One year after the posttest six nurses were left. None of the remaining nurses had had contact with parents who had an asthma-booklet.

The data presented above, which came from the health care providers, was compared to data gathered among the parents as far as possible. Generally, this check on accuracy of the data revealed that care givers had accurately reported the contacts they had had, the times they referred patients and the ways they provided the information.

Care providers who had used the protocol were asked whether they had observed changes in their communication with the parents when parents had read the information before the discussion. The majority $(79 \%)$ perceived their interaction with patients to have changed. Most frequently, they stated that parents contributed more to the discussion than before the use of the protocol. Moreover, they believed that parents could more easily understand the verbal information provided.

\subsubsection{Cooperation between disciplines}

The protocol starts from the principle that the relevant health care providers should cooperate in providing information to parents. This means that, whenever relevant, parents should be referred to other disciplines. As might be expected, process evaluation results show that the GP, who is the coordinator of care, mostly initiates contact with other disciplines. From health care providers' data and parents' reports could be deduced that for 10 parents (20\%) another care giver besides the GP was involved in the asthma education. For these patients a home visit was carried out by the nurses who advised parents on how to recognize and control environmental factors (such as dust and humidity) that influence asthma. Five GPs involved the community nurses $(n=4)$ and/or the asthma nurse $(n=3)$. For four of these GPs this was the first time they hadi ever made. such a referral. In another five (patient) cases the community nurses consulted the GP as indicated in the protocol. For three of them this was, the first time they had taken this initiative. Furthermore, the community nurses were the only discipline who had conferred with the doctors in child health centers. It should be noted that the doctors of the pediatric health centers dicl not report taking any initiative to contact or refer to other disciplines. Overall, practitioners involved in the study who did not initiate contact with other disciplines at posttest in the past six months, stated that the child's symptoms provided no reason for referral at the time. Two of these GPs indicated that referral had already occured. Nevertheless, several GPs who did not refer patients indicated that they 
did tell parents that they could get support in the asthma self-management from the other disciplines.

The parent questionnaire revealed that nine respondents had received a home visit aimed at advising parents on how to recognize and control environmental factors (such as dust and humidity) that influence asthma. Twice, however, the adaptation visit wasi carried out by the GP. Seven times the procedure was carried out by the community nurse, who was sometimes accompanied by the asthma nurse. This last procedure was suggested by the protocol.

At one year follow-up the disciplines' reported intention to cooperate with each other with respect to providing asthma education remained positive.

\subsubsection{Impact and outcome evaluation}

Prior to analysis the variables of attitude, knowledge, self-efficacy, self-management behavior and state-anxiety were exarnined within each group through various SPSS- $X$ programs for accuracy of data entry, missing values and assumptions of multivariate analysis (Tabachnick \& Fidell, 1989). There were no multivariate outliers in both pre- and posttest data and the follow-up data at $a=.001$. Results of the evaluation of assumptions of normality, homogeneity of variance-covariance matrices and linearity were satisfactory. Box's M-test for homogeneity of dispersion matices prouuces F(15.10705) $=.85 ; \rho<.01$ ror the pretest data and $F(15,14947)=.96 ; p<.49$ for the posttest data. A multivariate analysis was performed to evaluate baseline comparability of parents in the study groups with respect to the pretest scores on the dependent variables listed in Table 2. Neither the multivariate $F(F(5,58)=.49 ; p<.78)$ nor the univariate $F^{\prime}$ s revealed significant differences on the scores between treatment and control groups. The latter is indicated in Table 2 and will be explained hereafter. Assumptions for analysis of covariance were tested for the health status indicators. The evaluation results were satisfactory.

Univariate $F^{\prime}$ 's or chi-square statistics were studied with respect to the pretest data on medical care utilization and asthma severity variables. It needs to be emphasized that here another control group was involved than the one used as waiting list control group in the randomized trial. Findings are presented in Table 4. These findings suggest that the study groups were largely equivalent with regard to the dependent variables prior to the intervention.

At pretest the multiple correlation $(R)$ of knowledge, attitude and self-efficacy with self-management behavior was .50 . This means that the three variables together explained $25 \%$ of the variance for self-management behavior. The intercorrelations between the three variables were moderate. Self-efficacy correlated .33 with attitude and .32 with knowledge. The attitude correlated .49 with knowledge. 
Table 2

Means and standard deviations by group at pretest and posttest with general practitioners as unit of analysis; F-and p-value for univariate interaction effects "condition x time". Means and standard" deviations of the experimental group at posttest and one year follow-up with GPS as unit of analysis; t-and p-value.
Control group $(N=32)$
Experimental group $(N=29)$

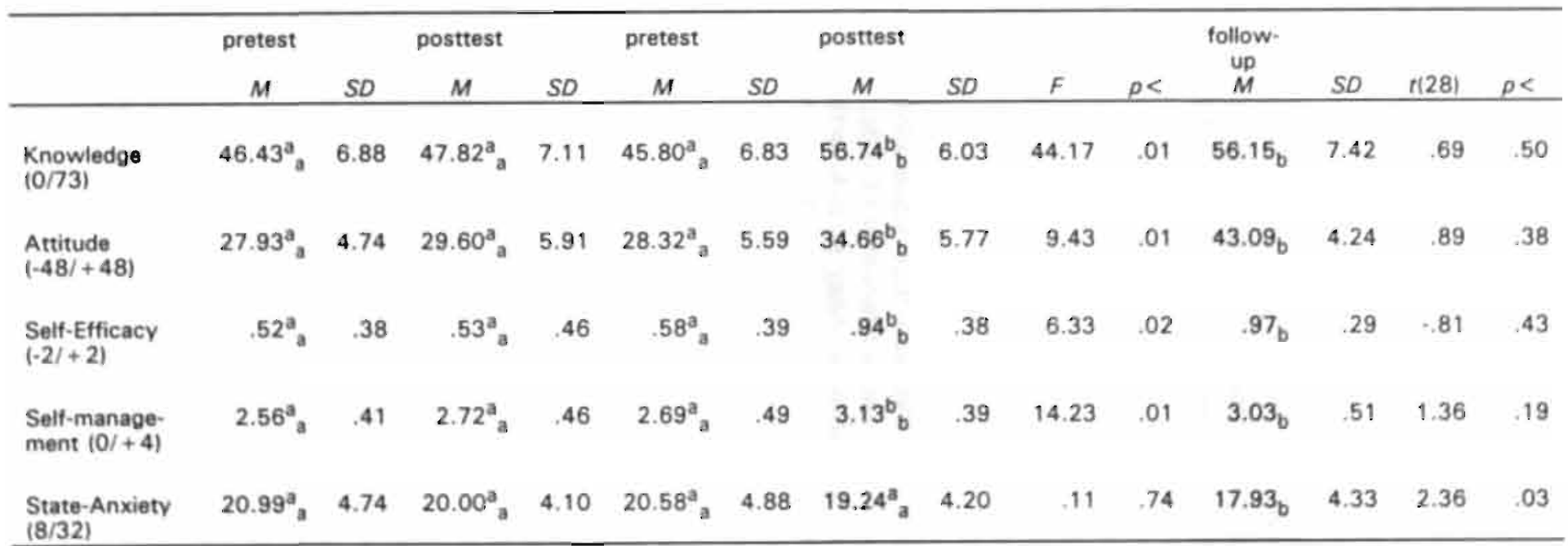

Note. For comparison of pretest and posttest columns per study group land the posttest and follow-up columns for the experimental group. only). the meanscores that do not share the same subscript significantly differed from each other at $\rho<.05$. For comparion of pretest columns between study groups. and likewise posttest columns between study groups, the mean score that do not share the same superscripts significantly differed from eachother at $p<.05$. 


\subsubsection{Impact evaluation: Educational and behavioral variables}

Analysis of the differences in the behavioral variables across time (pre- to posttest) between treatment and control group revealed effects for time $(F(5,58)=18.78$; $\rho<.01)$ and condition $(F(5,58)=2.96 ; p<.02)$. However, these main effects are to be explained in the light of a significant interaction effect for the dependent variables $F(5,58)=9.63 ; p<.01^{1}$. The difference revealed in effect is in favor of the treatment condition.

Univariate findings indicate that subjects in the treatment group improved significantly more than the control group in their knowledge about asthma, their attitude towards asthma, their perceptions of self-efficacy and their self-management behaviors. Level of state-anxiety did not change significantly at posttest. At one year follow-up pairwise t-tests revealed that improvements on the behaviora! variables were sustained for the experimental group (all $p>.18$ ). Furthermore, level of state-anxiety appeared to have decreased significantly at follow-up $(p<.03)$. These changes are discussed in further detaii. Pretest and posttest data are compared between the two study groups (manova), indicated in Table 2 by the superscripts. Pre- to posttest scores and posttest to follow-up scores were compared, these are indicated by the subscripts, within each group separately ( $t$-tests).

Stepwise regression, based on the predictive value of the predictors, was employed to determine the best linear combination of independent variables to predict gain in self-management behavior. After step 1, with gain in knowledge appearing in the equation, $R^{2}=.10, F(1,91)=10.39, p<.01$. After step 2 , gain in self-efficacy came into the prediction of gain in (reported) self-management behavior, $R=.38, R^{2}=.14$ (adjusted $R^{2}=.13$ ), $F(1,91)=7.13, \rho<.01$. Gain in attitude did not contribute significantly to regression.

More detailed analysis was carried out to find out which items or subscales of the overall scales contributed to the significant changes in knowledge, attitude, selfefficacy and self-management behavior.

Table 2. shows that parents in the treatment group gained more knowledge about asthma than those in the control group. The contribution of the specific modules to greater knowledge was studied by analyzing in what areas parents' knowledge improved (Table 3). For this purpose the knowledge items were related to the different topics or modules of the program. In all, 16 modules could be distinguished. For modules 10 and 16 no related knowledge items were incorporated into the questionnaire because these modules only elaborated on knowledge presented in other modules. Analysis was carried out with those parents in the treatment group who actually received a particular module. This was done to avoid committing a

\footnotetext{
'Results with practices; $(N=60)$ as unit of analysis revealed nearly identical results to the multivariate analysis with general practitioners as unit of analysis: multivariate $F(5,55)=9.63$; $\rho<.01$. Univariate findings on the practice level were also comparable to the univariate results with physicians as unit of analysis. Furthermore, results with GPs as unit of analysis and every attitude item separately in the analysis revealed the following interaction effect: $F(28,25)=2.40 ; p<.01$.
} 
type three error: evaluating a program or parts of a program that have not been implemented (Basch, Sliepcevich, Gold, Duncan \& Kolbe, 1985).

Table 3 indicates that the two groups did not differ on more general knowledge on asthma (modules 1 and 3-6), except for the module on "the working of the lungs.". Parents in the experimental group improved more than the control group on knowledge items that are related to this particular module. Table 3 shows that greater gain in knowledge in the treatment group was mainly effected by modules dealing with more applied knowledge such as how to monitor asthma attacks, how to keep your home adapted or how to use and interpret peakflow measurements.

To check for the possibility that the greater increase in knowledge in the experimental group was caused by factors other than the program, the two groups were compared with respect to the number of people who participated in other educational activities during the intervention period, such as participation in educational meetings and reading brochures, books or magazine articles on asthma. Chi-square statistics $\left(\chi^{2}(1, N=94)=.23 ; p<.63\right)$ revealed that the number of people participating in such activities was not significantly different in the study groups. In both groups about $39 \%$ of the respondents participated in other activities which mainly consisted of reading written materials.

Long term findings showed that the level of knowledge at posttest were maintained at one year follow-up. Item analyses revealed that the latter level of knowledge, which is the sum of the number of correct responses, was largely established by the same questions that were answered correctly at the posttest.

Improvements from pre- to posttest occurred in the attitude items related to medication. Parents in the treatment group agree less with statements like "I don't believe that medicines really help to make symptoms go away", and "Missing a dose won"t hurt". The treatment group agreed more strongly with items such as "It's important to take medicines on time". These questions, which were designed to assess the perception of parents' attitude towards compliance with medications, indicate a stronger belief in the necessity' of medication in the experimental group at posttest. At one year follow-up the attitude score was sustained.

Program effects on self-efficacy with regard to the prevention, treatment and monitoring behaviors were studied. Three GPs of the control group $(n=29)$ were excluded from the analysis because of missing data on one of the subscales. Univariate findings showed that parents in the treatment group indicated a significantly larger improvement on self-efficacy with respect to prevention $(F(3,57)=12.09$; $p<.01)$ and treatment $(F(3,57)=16.43 ; p<.01)$ than the control group. Overall, parents in the treatment group improved on every item of the prevention and treatment self-efficacy scale, but only significantly more than the control group with respect to the items "Ability to take preventive actions against pollen" or "Temperature changes within the home" and "Taking preventive actions when the weather is moist". Furthermore, parents in the treatment group had significantly more confidence than the control group in their ability to treat severe asthma attacks and to estimate the correct doses of medication. 
Table 3

Means on knowledge per module, at pre-and posttest, for the treatment and control groups separately.
Experimental group
Control group
$(N=26-44)$

$$
(N=47)
$$

\begin{tabular}{|c|c|c|c|c|c|c|c|c|}
\hline & Range & $\begin{array}{l}\text { Pretest } \\
\qquad M\end{array}$ & $\begin{array}{l}\text { Posttest } \\
\mathbf{M}\end{array}$ & $n$ & $\begin{array}{l}\text { Pretest } \\
\qquad M\end{array}$ & $\begin{array}{l}\text { Posttest } \\
M\end{array}$ & $F$ & $p<$ \\
\hline $\begin{array}{l}\text { 1. What is } \\
\text { asthma }\end{array}$ & $(0-4)$ & 1.50 & 2.23 & 44 & 1.60 & 1.94 & .93 & $\mathrm{~ns}$ \\
\hline $\begin{array}{l}\text { 2. Working } \\
\text { lungs }\end{array}$ & $(0-6)$ & 2,50 & 3.75 & 44 & 2.68 & 2.75 & 9.39 & .01 \\
\hline 3. Allergy & $(0-12)$ & 8.76 & 9.69 & 42 & 8.57 & 8.87 & 1.73 & ns \\
\hline $\begin{array}{l}\text { 4. Hyperreac- } \\
\text { tivity }\end{array}$ & $(0-8)$ & 6.29 & 7.29 & 42 & 5.72 & 6.28 & 1.29 & ns \\
\hline 5. Infections & $(0-8)$ & 5.29 & 6.31 & 42 & 5.23 & 6.00 & .23 & ns \\
\hline $\begin{array}{l}\text { 6. Internal } \\
\text { factor }\end{array}$ & $(0-12)$ & 4.48 & 7.24 & 42 & 4.72 & 6.23 & 2.78 & ns \\
\hline 7. Medication I & $(0-7)$ & 3.82 & 5.74 & 38 & 3.57 & 3.81 & 9.98 & .01 \\
\hline 8. Inhalers I & $(0-2)$ & .92 & 1.56 & 36 & .72 & .66 & 11.80 & .01 \\
\hline 9. Inhalers II & $(0-3)$ & .47 & 1.14 & 36 & .36 & .30 & 10.15 & .01 \\
\hline $\begin{array}{l}\text { 10. Medication } \\
\text { II }\end{array}$ & 10.21 & & & 36 & & & & \\
\hline 11. Peak flow & $(0-2)$ & .29 & 1.32 & 38 & .15 & .13 & 36.84 & .01 \\
\hline 12. Monitoring & $(0-18)$ & 8.78 & 12.15 & 40 & 8.83 & 9.26 & 11.81 & .01 \\
\hline 13. Advice & $(0-4)$ & 3.00 & 3.60 & 37 & 2.98 & 3.00 & 4.05 & .05 \\
\hline $\begin{array}{l}\text { 14. Adapting } \\
\text { homes }\end{array}$ & $(0-6)$ & 4.30 & 5.16 & 37 & 4.11 & 4.17 & 6.29 & .02 \\
\hline 15. Report & $(0-27)$ & 15.56 & 19.00 & 32 & 15.17 & 16.49 & 5.20 & .03 \\
\hline $\begin{array}{l}\text { 16. Medication } \\
\text { chart }\end{array}$ & & & & 26 & & & & \\
\hline
\end{tabular}

Nore. The $p$-value of a test of interaction effect "condition $x$ time" is indicated. The $n$ for the treatment group indicates the number of respondents that received the module in question and had no missing data on the relevant questions (for that module). 
Finally, besides the overall self-efficacy score, the subscales for self-efficacy were compared at posttest and one year follow-up. $T$-statistics did not reveal a significant overall score nor significant differences per subscale, indicating that the gain in selfefficacy was sustained also with respect to prevention, monitoring and treatment of asthma.

Self-management behavior was considered the major impact variable in the study. The analysis of the subscales of the self-management scale involved 28: GPs of the control group and 29 GPs of the treatment group. Five GPs were omitted from the analysis because of missing data on one of the subscales. Multivariate analysis indicates a significant improvement in all the subscales of self-management except for the treatment behavior scale. The mean scores on the pre- to post-behavioral prevention scale (univariate analysis: $F(3,54)=4.73 ; p<.04$ ) and monitoring behav ioral scale (univariate analysis: $F(3,54)=4.73 ; p<.03$ ) improved more in the experimental than the control group as a result of an improvement on all items. Improvements were maintained at one year follow-up. Specific $t$-statistics per subscale are included in Appendix 4.

Reported change in behavior may occur because parents report performing behaviors more frequently and/or report performing more different types of behaviors. The total number of reported behaviors at pretest and posttest was computed with parents as unit of analysis. Repeated measures manova revealed no significant differences between treatment and control group with regard to the number of behaviors parents performed $(F(2,93)=.38 ; p<.54)$. This implies that improvement in behavior might be ascribed mainly to an increase in performance frequency.

At one year follow-up the number of behaviors performed was not significantly different from the number measured at posttest, $t(44)=-.82, p<.42$. Also, no significant differences were revealed at the level of the subscales.

According to Van der Ploeg et al. (1980), one might except a normal mean score of 13.3 on the state-anxiety scale. This is the score which was found in a population of women students in a non-frightening situation. Table 2 showed that the mean state-anxiety score was about 20 for both groups of parents on both preand posttest. This means that parents in this study, who were instructed to imagine their child's asthma attacks, experienced high levels of state-anxiety when such an event occurred. At one year follow-up, the level of state-anxiety appears to have decreased significantly. This indicates that one year after the posttest, parents in the experimental group seem to be less anxious when reporting their response to their child's asthma-attack.

\subsubsection{Sociodemographic variables}

Relationships between the gain scores and several sociodemographic variables were analyzed to find out whether the program was especially effective for people (parents, children and health care providers) with a specific sociodemographic background. Type of GPs' practice (solo, duo or group practice) did not seem to 
have influenced the gainscores (from pre- to posttest) in either the treatment $(F(5$, $26)=.42 ; p<.93)$ or control group $F(5,27)=1.72 ; p<.11$ ).

With patients as unit of analysis, correlations were obtained between the gainscores and child's age, duration of disease, time since diagnosis and time since treatment, educational background of the parents and the extent to which asthma was considered a problem by parents. These correlations were computed for treatment and control group separately. For the treatment group only a few significant correlations were revealed. Firstly, the higher the level of education of the respondents, the greater their gain in attitude $\left(r_{s}=.29 ; p<.03\right)$. Secondly, respondents who considered asthma as a greater problem gained more in knowledge $\left(r_{\mathrm{s}}=.29 ; p<.04\right)$. Furthermore, the higher the respondents' age the greater the gain in knowledge $(r=.25 ; \rho<.05)$ and self-efficacy $(r=.26 ; p<.04)$. No significant correlations were revealed for the control group.

\subsubsection{Outcome evaluation: Health status indicators}

Two time periods were distinguished. Period 1 is the year preceding the pretest and period 2 is the year after the posttest. Analysis of covariance was conducted to find out whether the experimental group outperformed the control group on the data collected in period 2 by more than would be expected on the basis of the data of period 1. Table 4 shows that the number of non-emergency and emergency contacts between the GP and the parents because of asthma decreased significantly more in the experimental group as compared to the control group. A slight (although not significant at $p<.05$ ) decrease in hospital admissions was revealed for the experimental group but not for the control group. To validate the data presented by GPs in the experimental group, they were compared with data provided by the parents in the experimental group. Analyses ( $t$-tests) revealed similar findings. A significant deviation was revealed with respect to the number of emergency contacts with the GPS. According to the parents relatively more emergency contacts with the GPs took place than was reported by the GPs themselves, respectively in period $1 t(15)=2.90, p<.02$ and in period $2 t(15)=2.71, p<.02$. Furthermore, parents reported more contacts with a specialist in both period 1 and 2 when compared with the data provided by the $\mathrm{GP}$, respectively $t(10)=3.02, p<.02$ and $t(10)=2.46, p<.04$.

To get an indication of asthma severity in each group for both periods, the following variables were analyzed for duration of asthma episodes and asthma severity of asthma symptoms as estimated by the GP. With respect to the variable "duration of episodes" GPS could choose one out of four options: the duration of opisodes has increased in time, decreased, not changed, or episodes have not occurred. The category which indicates a decrease in duration when comparing period 2 to period 1 was compared with all other categories combined (no difference, no symptoms in both periods and duration of periocls increase from period 1 to period 2). Chi-square statistics revealed that GPs in the experimental group reported more often than the control group that the duration of the asthma symptoms 
Table 4

Means and standard deviations of medical care utilization variables by group of patients in period 1 and period $2 ; F$-and $p$-value for covariance effects. Univariate findings by proup in period 1 (1) year) and period 2 (1 year)
Experimental group
$(N=28)$
Control group
$(N=18)$

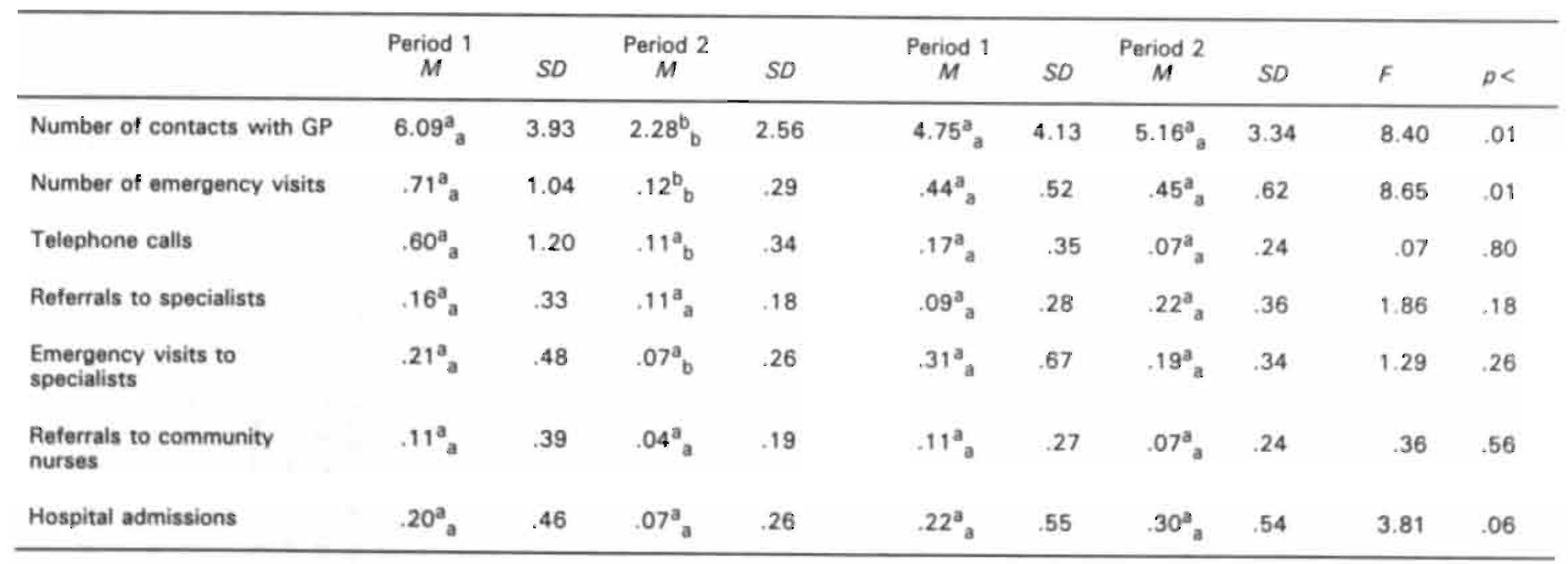

Note. For comparison of period 1 with period 2 columns per study group, the meanscores that do not share the same subscripts significantly differed from each other at $p<.05$. For comparison of the period 1 columns between study groups and likewise the period 2 columns between study groups. the mean scores that do not share the same superscripts differed from each other at $p<.05$. 
had become less in period 2 compared to period $1, \chi^{2}(1, N=46)=5.60, p<.02$. Finally, covariance analysis revealed that the estimated asthma severity had decreased more in the experimental group than in the control group, $F(2,43)=$ $14.87, p<.01$. Perceived asthma severity at pretest was analyzed for the experimental group with respect to the gain in behavioral variables from pre- to posttest. No difference was revealed between the distinguished subgroups, multivariate $F(5,41)=70.0, p<.37$ (interaction effect), implying that the beha vioral outcomes are not different for different levels of asthma severity.

\subsection{Discussion}

The present study examined the effectiveness of a patient education protocol for parents of young children (0-4 years) with asthma. For this purpose four disciplines were expected to provide coordinated care and education in primary health care. The asthma protocol can be considered as an effective intervention to positively guide and maintain parents' knowledge, attitudes, perceptions of self-efficacy, state-anxiety, and even more important to change and sustain their self-management behavior. Furthermore, the protocol appears to have a beneficial influence on registered medical care utilization and perceived asthma severity. Both health care providers and parents placed a high value on the educational materials and it seems that the disciplines involved take a positive attitude towards cooperation regarding the use of the protocol.

The current study showed that two factors seemed to be especially responsible for the gain in self-management behavior, namely gain in knowledge and gain in self-efficacy. Social leaning theory provides support for these findings. This theory (Bandura, 1986) suggests that behaviorall capability (which includes knowledge of what to do and how to do it) and self-efficacy mediate behavior. Parents in this study improved especially their applied knowledge and their confidence in their ability to manage asthma. The reported improvement in the frequency in which behaviors are performed seems to indicate that the program was indeed successful in changing self-management behaviors. In addition, the results suggest improvement in medical care utilization and severity of asthma symptoms las perceived by GPsi, another indicator of actual behavior change.

It is not surprising that the parents in the treatment and control group did not differ significantly on more general aspects of asthma, because it may be expected that people who have known for some time that their child has asthma, will have already gathered some information on what asthma is and the role allergy, hyperreactivity, infections and some internal factors like emotion and exercise have in causing asthma.

Although the control group did not receive information from the protocol, their knowledge improved slightly. Some of this increase in the control group might have been caused by instrument reactivity. This means that at least some of the improvement in knowledge in the control group might be related to an increased 
awareness, in parents of their child's disease, as a result of answering the pretest questionnaire (Van Asperen et al., 1986). Parents may have been stimulated to. gather information after completion of the pretest questionnaire. Especially the information in the first modules is readily available in popular literature.

On the other hand, parents in the experimental group might have been more compelled than the control group to report "appropriate" management behaviors because their care providers have advised them to engage in these management behaviors at the time that they discussed the asthma information. To avoid this kind of misrepresentation, parents were assured at the start of the study that all the information provided would never be passed on to their care providers and that the. reported data would only be published on group level. This was done to ensure anonymity which is, according to Murray and Perry (1987), an essential prerequisite. to reduce the perceived pressure to provide invalid responses to self-report measures. Furthermore, in this study the changes in the behavioral variables were. validated by the demonstrated benefits of the experimental group of health outcomes such as reduced health care utilization and reduction of asthma severity.

Negative attitudes toward medication are considered of direct relevance to noncompliance (Evans \& Spelman, 1983). Results showed that parents in the treatment group acquired a more positive attitude to medication. This finding indicates that psychological resistance to medication may be amenable to education. programs directed at modifying attitudes.

A reassuring finding is that more responsibility allocated to parents did not result in higher levels of anxiety. One might be tempted to expect a lowered level of anxiety as a consequence of heightened self-efficacy at posttest. However, other studies (Bandura, 1988) suggest that feelings of anxiety may exist independently of perceived self-efficacy.

Furthermore, no strong relationships were found between sociodemographic variables and changes in knowledge, attitude, self-efficacy and self-management behaviors. This means that the program does not seem to be especially effective for only some sociodemographic groups. Furthermore, it might be concluded that our enrolment criteria for the parents, with respect to time of diagnosis, might have been unnecessarily strict, because it appeared that time of diagnosis had no important influence on the effect measures.

Evaluation data indicated that cooperation between the disciplines involved generally took place as suggested in the protocol, though some deviations were observed. Nevertheless, this conclusion has to be presented with some caution, as we had to rely on the care givers' judgement whether referral or cooperation was needed. However, the low rate of referral does not mean that parents received incomplete asthma education. It needs to be repeated that the GP is responsible for providing the information to parents. To reduce their work load, the GPs are recommended to involve other disciplines to discuss the information provided to parents. As reported, the modules were distributed to a satisfactory degree.

In general, most health care providers in the organizations for home care do not use the protocol on a routine basis. Lack of contact with parents who have a 
parent-booklet seems to be a reason for this observation. As a community nurse indicated, "Up to now I am the only one in our organization who has the educational materials, so there is little chance that a parent will have contact with a nurse who is familiar with the asthma materials. Furthermore, not all GPs in our region were involved in the study. Therefore, a lot of our patients have never heard of the asthma materials". From the data of the disciplines involved we might infer that, when implementing the protocol, we need to involve all relevant health care providers in a whole district to guarantee continuity of care and education.

The lack of initiative of the doctors working at the child health care centers may be explained by the fact that according to the protocol their main task was to detect children with asthma and subsequently refer them to the GP for the confirmation of the diagnosis. Since the patients in this study had already been diagnosed, the main task of this discipline did not need to be performed.

Response rates in the telephone survey and written survey among employees of the organizations for home care were moderate. Non-response in many cases was largely caused by practical limitations. Many employees within the organizations for home care work part-time, in different locations or cannot be easily disturbed during working hours. Furthermore, the community care organizations appear to have a considerable turnover of work force. On the other hand, it is possible that health care providers who had not worked with the protocol did not appreciate it and did not feel like investing time in evaluating the program. However, findings indicated that both the care providers who did use the materials and those who did not were equally enthusiastic about the materials. This indicates that barriers (such as "no parents with booklets seen") exist that hinder actual performance. Overall, health care providers' perceptions of the format, the convenience for use and value of the protocol for their discipline appeared to be very favorable. Although favorable perceptions alone might not be sufficient, it is one of the factors necessary to facilitate program implementation (Basch et al., 1985).

It might be concluded that at posttest the health care providers most involved followed the suggestions of the asthma protocol with respect to the distribution and subsequent discussion of the information with parents. Health care providers' reports did largely correspond with patients' responses to these aspects of the transfer of the information. At one year follow-up, more GPs handed out the complete booklet during a single consultation. This is not suggested in the protocol. However, in our view, discussing the information in several contacts is more important than the proportional distribution of the parent-booklet.

The following methodological issues in the present study need to be considered. The lack of a control group with respect to the determination of the long term effects on the behavioral variables might not be a severe shortcoming. These variables did differ significantly between the experimental and control group at an earlier point in time (at posttest); the follow-up survey did not show any regression on the behavioral variables in question. Findings on the behavioral variables have been based on seli-reports from parents. To control for effects such as the haloand Hawthorne effects (Spector, 1981), more "objective" medical data were collect- 
ed from patients medical records. Variables such as contact with the GPs appeared to be well documented (standard), as well as referral to the specialist and hospitalizations. However, this did not apply to some of the other variables measured. Whether other variables like telephone calls are registered depends on the kind of insurance of the patient. For national insured patients GPs receive a fixed budget for one year, so there is no need with these patients to keep a standard registration for telephone calls. When filling out the questions on asthma severity and leven more) when filling out findings on duration of asthma we had to rely on GPs opinions. One can not ban the occurrence of socially desirable answering in this respect. However, GPs in the control group might also be tempted to make a good impression. Furthermore, there appeared to be a problem with the interpretation among parents and GPs on whether a contact was an emergency or not. As might be expected, parents labeled more contacts as urgent than the GP. The fact that patients reported a higher frequency of contacts with the specialist might be a result of the way of questioning. In the survey GPs were asked to indicate the number of referrals and parents were asked to report the number of contacts. The latter might also have included regular check-up contacts without any referral from the GP.

Apparently hospital admissions and consulting specialists did not occur frequently. Furthermore, the majority of the patients did not seem to be under (continuous) treatment of a specialist. These findings are consistent with the type of patients one might expect to be treated in primary care.

Especially with a design which does not have controlled assignment of subjects to an experimental and a control group, one might still wonder whether the findings on medical care utilization and asthma severity may be a result of differences between the subjects in the study groups. However, sociodemographic variables and pretest data on the patients' asthma severity and medical care utilization were largely comparable in both study groups. Furthermore, in this study we have found no indications that the treatment of asthma was influenced by the type or size of general practice. Finally, both study groups were informed about our intention to collect earlier mentioned data after the data collection period had passed in order to avoid that GPs would change their way of registration as a result of knowing our intentions.

In our study the internal consistency of the attitude survey was low. This was not considered to be that important because we have to measure several different asthma facets which were not expected to be strongly related to each other.

Some of the patients in the current study were no longer eligible for data collection because they had changed GPs. The percentage of loss in this study did not deviate from the average percentage (10-15\%) of people in the Netherlands who change GPs on a yearly basis (Schouwstra, 1976).

It might be concluded that the asthma protocol realizes results which are comparable to several American asthma programs, which also seem to be effective in altering behavioral variables and medical care utilization of people with asthma (Klingelhofer \& Gerswin, 1988). However, such similarities should be interpreted 
with care. The American health services are organized differently from the services in the Netherlands. There is, for example, a higher mobility among patients in the USA and there appears to be more competition between the practices of GPs on one side and the hospitals on the other. GPs as we know them are rarely found in the USA. Furthermore, patient education is a domain where cultural differences play an important part (Kanters, 1988). At any rate, the protocol seems to work for the Dutch situation and provides positive effects on both short term and long term. The future course of the project is aimed at dissemination of the protocol to a larger population.

\subsection{Implications for practice}

Perhaps the most important implication for piractice is that now there is an effective education program available for preschool children with asthma which can be used during regular medical (primary) care. Resuits further suggest that differentiation between knowledge which is read and absorbed and which requires action seems to be crucia!. Parents who read written information before contact with the care providers are stimuiated to be involved during the contact.

The results of the project also demonstrate the usefulness of combining an educational protocol for use by the health care providers and educational material for the parents of the asthmatic children. In many cases patient education is attempted by providing educational materials only to the patients. The protocol gives the health care providers specific steps to follow and suggestions as to which teaching methods to use. In our study we found that the practitioners were receptive to the use of the protocol, and their use of it facilitated implementation of the patient education program. 


\section{References}

Asperen, P., van, Jandera, E., Neef, J., de, Hill, P., \& Law, N. (1986).

Education in childhood asthma: a preliminary study of need and efficacy. Australian Paediatric Journal, 22, 49-52.

Basch, C. E., Sliepcevich, E. M., Gold, F. S., Duncan, D. F., \& Kolbe, L. I. (1985). Avoiding type III errors in health education program evaluations: a case study. Health Education Quarterly, 12, 315-331.

Basch, C. E. (1987). Focus group interview: an underutilized research technique for improving theory and practice in health education. Health Education Quarterly, $14,411-448$.

Bandura, A. (1977) Social Learning Theory. Englewood Cliffs: Prentice-Hall.

Bandura, A. (1986) Social foundations of thought and action, a social cognitive theory. Englewood Cliffs: Prentice-Hall.

Bandura, A. (1988). Self-efficacy conception of anxiety. Anxiety Research, 1 , 77-98.

Bartholomew, L. K., Parcel, G. S., Seilheimer, D. K., Czyzewski, D., Spinelli, S. H., \& Congdon, B. (1991). Development of a health education program to promote the self-management of cystic fybrosis. Health Education Quarterly, 18, 429-443.

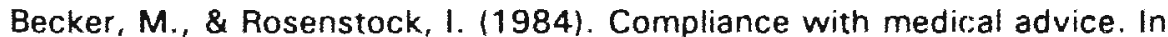
A. Steptoe, \& A. Matthews (Eds.), Health care and human behavior (pp. 175 208). New York: Academic Press.

Berg, B. K., Eckhoff-Biagi, P., Hebert, P., Rodell, D., \& Sprafkin, R. (1987). Patient education needs assessment: constructing a generic guidf: Patient Educa tion \& Counseling, 9, 199-207.

Bremberg, S. G., \& Kjellman, N. I. M. (1985). Children with asthma: how do they get along at school? Acta Paediatric Scandinavia, 74, 833-840.

Brink, W. P., van den, \& Koele, P. (1987). Statistiek. Deel 3: Toepassingen. 
Meppel: Boom.

Bruhn, J. G. (1983). The application of theory in childhood asthma self-help programs. The Journal of Allergy \& Clinical Immunology, 72, 561-577.

Busse, W. W., \& Reed, Ch. E. (1988). Asthma: Definition and pathogenesis. in E. Middleton, Ch. E. Reed, E. F. Ellis, N. F. Adkinson, \& J. W. Yunginger (Eds.), Allergy, principles and practice (pp. 969-991). St. Louis - Washington DC.Toronto: The C.V. Mosby Company.

Campbell, D. T., \& Stanley, J. C. (1966). Experimental and quasi-experimental designs for research. Chicago: Rand McNally College Publishing Company.

Centraal Bureau voor Statistiek (1986). Compendium Gezondheidsstatistiek Nederland. 's-Gravenhage: Staatsuitgeverij.

Centraal Bureau voor Statistiek (1989a). Ontslagen ziekenhuispatiënten naar hoofddiagnose, leeftijd en geslacht in het jaar 1987. Voorburg: Staatsuitgeverij.

Centraal Bureau voor Statistiek (1989b). Overledenen naar doodsoorzaak, leeftijd en ges/acht in het jaar 1988. Voorburg: Staatsuitgeverij.

Clark, N. M., Feldman, C. H., Fieudenberg, N., Millman, E. J., Wasilewski, Y., \& Valle, I. (1980). Developing education for children with asthma through study of self-management behavior. Health Eolucation Quarterly, 7. 278-297.

Clark, N. M., Feldman, C. H., Evans, D., Millman, E. J., Wasilewski, Y., \& Valle, I. (1981). The effectiveness of education for family management of asthma in children: a preliminary report. Health Education Quarterly, 8, 166-174.

Clark, N. M., Feldman, C. H., Evans, D., Duzey, O., Levison, M. J., Wasilewski, Y., Kaplan, D., Rips, J., \& Mellins, R. B. (1986). Managing better: children, parents, and asthma. Patient Education \& Counseling, 8, 27-38.

Clark, N. M., Feldman, C. H., Evans, D., Levison, M. J., Wasilewski, Y., \& Mellins, R. B. (1986). The impact of health education on frequency and cost of health care use by low income children with asthma. The Journal of Allergy \& Clinical Immunology, 78, 108-115.

Clark, N. M. (1989). Asthma self-management education, research and implications for clinical practice. Chest, $5,1110-1113$.

Clark, N. M., \& Zimmerman, B. J. (1990). A social cognitive view of selfregulated learning about health. Health Education Research, 5, 371-379.

Colland, V. (1988). Ontwikkelingspsychologische aspecten bij astma. De beleving van de ziekte bij het opgroeiende kind. Kind \& Adolescent, 9, 85-97.

Colland, V. T. (1990). Leren omgaan met astma, interventiemethoden voor kinderen en ouders (proefschrift). Amsterdam: Vrije Universiteit.

Conway, S. P., \& Littlewood, J. M. (1985). Admission to hospital with asthma. Archives of Disease in Childhood, 60, 636-639.

Creer, Th. L., Rienne, C., \& Christian, W. (1976). Behavioral contributions to rehabilitation and childhood asthma. Rehabilitation Literature, 37, 226-232.

Creer, Th. L., \& Winder, J. A. (1986). Asthma. In K. A. Holroyd, \& Th. L. Creer, (Eds.) Self-management of chronic disease. Handbook of clinical interventions and research (pp. 269-203). London: Academic Press Inc.

Creer, Th. L., Wigal, J. K., Tobin, D. L., Snyder, S. E., \& Winder, J. A. (1986). 
An asthma attitude scale for adults. Athens: Self-management Systems.

Creer, Th. L. (1987). Living with asthma: Replications and extensions. Health Education Quarterly, 14, 319-331.

Creer, Th. L., \& Kotses, H. (1989). Asthma. In Th. H. Ollendick, \& M. Hersen (Eds.) Handbook of child psychopathology (pp. 341-357). New York: Plenum Publishing Corporation.

Creer, Th. L., \& Kotses, H. (1990). An extension of the Reed and Townley conception of the pathogenesis of asthma: The role of behavioral and psychological stimuli and responses. Pediatric asthma, Allergy \& Immunology, 4, 81-104.

Creer, Th. L., Wigal, J. K., Kotses, H., \& Levvis, P. (1990). A critique of 19 self-management programs for childhood asthma: part II. Comments regarding the scientific merit of the programs. Pediatric Asthma, Allergy \& Immunology, 4, 41-55.

Deaton, A. V. (1985). Adaptive noncompliance in pediatric asthma: the parent as expert. Journal of Pediatric Psychology, 10, 1-14.

Dekkers, F. (1981). Patiëntenvoorlichting: De onmacht en de pijn. Baarn: Ambo.

Desvousges, W. H., \& Smith V. K. (1988). Focus groups and risk communication: the "science" of listening to data. Risk Analysis, 8, 479-484.

Dirksen, W. J., Geyer, R. M. M., Haan, M., de, Kolnaar, B. G. M., Merkx, J. A. M., Romeijnders, A. C. M., Dijkstra, R. H., \& Laar, J. R., van der (1992). NHGstandaard astma bij kinderen. Huisarts \& Wetenschap, 35, 355-3.62.

Donnelly, J. E., Donnelly, W. J., \& Thong, Y. H. (1987). Parental perceptions and attitudes toward asthma and its treatment: a controlled study. Social Science \& Medicine, 24, 431-437.

Dodge, R., Cline, M. G., \& Burrows, B. (1986) Comparisons of asthma, emphysema, and chronic bronchitis diagnoses in a general population sample. American Review of Respiratory Disease, 133, 981.986.

Ellis, E. F. (1983). Asthma in childhood. The Journal of Allergy \& Clinical Immunology, 72, 526-539.

Ellis, M. E., \& Friend, J. A. R. (1985). How well do asthma clinic patients understand their asthma? British Journal of Disease of the Chest, 79, 43-48.

Evans, R., Mullally, D. I., Wilson, R. W., Gergen, P. I, Rosenberg, H. M., Grauman, J. S., Chevarley, F. M., \& Feinleib, M. (1987). Prevalence hospitalization and death from asthma over two decades: 1965-1984. Chest, 91, (6S), 655-745.

Evans, D., Clark, N. M., Feldman, C. H., Rips, J., Kaplan, D., Levison, M. J., Wasilewski, Y., Levin, B., \& Mellins, R.B. (1987). A school health education program for children with asthma aged 8-11 years. Health Education Qwarterly, 14, 267-279.

Evans, L., \& Spelman, M. (1983). The problem of non-compliance with drug therapy. Drugs, 25, 63-76.

Feldman, C. H. (1987). Asthma education: general aspects of childhood programs. The Journal of Allergy \& Clinical Immunology, 80, 494-497.

Fergusson, D. M., Horwood, L. J., \& Shannon, F. T. (1983). Parental asthma, 
parental eczema and asthma and eczema in early childhood. Journal of Chronic Disease, 36, 517-524.

Fern, E. F. (1982). The use of focus groups for idea generation: the effects of group size, acquaintanceship,.and moderator on response quantity and quality. Journal of Marketing Research, 19, 1-13.

Folch-Lyon, E., \& Trost, J. F. (1981). Conducting focus group sessions. Studies Family Planning, 12, 443-449.

Geradts, W. F. (1987). Het consultatiebureau in grote en nieuwe lijnen. Bunnik: Nationale Kruisvereniging.

Gerritsen, J. (1989a). Prognosis of childhood asthma (Thesis). Assen/ Maastricht: Van Gorcum.

Gerritsen, J. (1989b). CARA bij Kinderen, Diagnostiek en Behandeling. Nieuwegein: Glaxo Pulmonaal.

Glik, D., Gordon, A., Ward, W., Kouame, K., \& Guessan, M. (1988). Focus group methods for formative research in child survival: an Ivoirian example. International Quarterly of Community Health Education, 8, 297-315.

Green, L. W., Kreuter, M. W., Deeds, S. D., \& Partridge, K. B. (1980). Health education planning, a diagnostic approach. Palo Alto: Mayfield Publishing Com. pany.

Green, L. W., \& Kreuter, M. W. (1991). Health promotion Planning, an educational and environmental approach. Mountain View: Mayfield Publishing Company.

Gregg, I. (1985). Epidemiology in asthma. In T. J. H. Clark, \& S. Godfrey (Eds.), Asthma (pp. 214-240). London: Chapman and Hall.

Heimann-Ratain, G., Hanson, M., \& Peregoy, S. M. (1985). The role of focus group interviews in designing a smoking prevention program. The Journal of School Health, 55, 13-16.

Hindi-Alexander, M. C., \& Cropp, G. J. A. (1984). Evaluation of a family asthma program. The Journal of Allergy \& Clinical Immunology, 74, 505-510.

Holman, H., \& Lorig, K. (1992). Perceived self-efficacy in de self-management of chronic disease. In R. chwarzer (Ed.), Self-efficacy: Thought control of action (pp. 305-324). Washington: Hemisphere Publishing Corporation.

Holroyd, K. A., \& Creer, Th. L. (1986). Self-management of chronic disease.

Handbook of clinical interventions and research. London: Academic Press Inc. Hoogen, H. J. M., van den, Huygen, F. J. A., Schellekens, J. W. G., Straat, J. M., \& Velden, H. G. M., van der (1985). Morbidity figures in general practice. Data from four general practices, 1978-1982. Nijmegen: Nijmeegs Universitair Huisartsen Instituut.

Huygen, F. J. A., Eijk, J., van, Hoogen, H., van den, Heeswijk, A., van, lersel, J., van, Roozen, R., Sechterberger, O., Vlaar, N., \& Wert, C., van de (1977). Een praktijk doorgelicht op CARA (I). Huisarts \& Wetenschap, 30, 383-386. Joos, S. K., \& Hickam, D. H. (1990). How health professionals influence health behavior: Patient-provider interaction and health care outcomes. In K. Glanz, F. M. Lewis, \& B. K. Rimer (Eds.), Health behavior and health education, theory, 
research, and practice (pp. 216-241). San Francisco/Oxford: Jossey-Bass Publishers.

Kanters, H. (1988). De effectiviteit van patiëntenvoorlichting. In A. Jonkers,

W. F. M. de Haes, G. J. Kok, P. C. Liedekerken, \&i J. A. M. Saan (Eds.), Effectiviteit vangezondheidswoorlichtingen-opvoeding (GVO) (pp. 97-120), Rijkswijk: Uitgeverij voor gezondheidsbevordering.

Kanters, H. \& Jonkers, R. (1988). Ontwikkeling en implementatie van modellen voor patiêntenvoorlichting: een verkennende analyse. In V. Damoiseaux, \& A. Ph. Vissers (Eds.), Patiëntenvoorlichting een interdisciplinaire benadering 1 pp. 118 132). Assen/Maastricht: Van Gorcum.

Kaplan, R. M., Chadwick, M. W., \& Schimmel, L. E. (1985). Social learning intervention to promote metabolic control in type I diabetes mellitus: pilot experiment results. Diabetes Care, 8, 152-155.

Kaptein, A. A. (1982). Iliness behavior of patients with asthma (thesis). Meppel: Krips Repro.

Kaptein, A. A., Dekker, F. W., Gill, K., \& Waart, M.A.C., van der (1987). Undertreatment of asthma in Dutch general practice. Family Practice, 4, 219 225.

Keller, K. L., Sliepcevich, E. M., Vitel\%, E. M., Lacey, E. P., \& Wright, W. R. (1987). Assessing beliefs about needs of senior citizens using the focus group interview: a qualitative approach. Health Education, 18, 44-49.

Kerrebijn, K. F., Hoogeveen-Schroot, H. C. A., \& Wal, M. C., van der (1976). CARA bij kinderen; een vervolgonderzoekgedurende 5 jaar. Nederlands Tijdschrift voor Geneeskunde, 120, 1030-1038.

King, J. (1983). Health beliefs in consultation. In D. Pendleton, \& J. Hasler (Eds.), Doctor-patient communication (pp. 109-125). London: Academic Press.

Kinsman, R. A., Spector, S. L., Shucard, D. W., \& Luparello, T. J. (1974). Observations on patterns of subjective symptomatology of acute asthma. Psychosomatic Medicine, 36, 129-143.

Klingelhofer, E. L. (1987). Compliance with medical regimens, self-management programs, and self-care in childhood asthma. ClinicalReview Allergy, 5, 231-247.

Klingelhofer, E. L., \& Gershwin, M. E. (1988). Asthma self-managemiant programs: premises, not promises. Journal of Asthma, 25, 89-101.

Kok, G., Vries, H., de, Mudde, A. N., \& Strecher, V. J. (1991). Planned health education and the role of self-efficacy: Dutch research. Health Education Research, 6, 231-238

Kok, G. J. (1988). Health motivation: Health education from a social psychological point of view. In S. Maes, C. D. Spielberger, P. B. Defares, \& I. G. Sarason (Eds.), Topics in health psychology (pp. 295-300). New York: Wiley.

Kok, G., Boer, D. J., den, Vries, H., de, Gerards, F., Hospers, H. J., \& Mudde. A. N. (1992). Self-efficacy and attribution theory in health education. In $R$. Schwarzer (Ed.), Self-efficacy: Thought control of action (pp. 245-262). Washington: Hemisphere Publishing Corporation.

Korhonen, T., Huttunen, J. K., Aro, A., Hentinen, M., Ihalainen, O., Majarder, 
H., Siitonen, O., Uusitupa, M., \& Pyorola, K. (1983). A controlled trial on the effects of patient education in the treatment of insulin-dependent diabetes. Diabetes Care, 6, 256-261.

Krueger, R. A. (1988). Focus groups: a practical guide for applied research. Newbury Park: Sage Publictions.

Lamberts, H. (1984). Morbidity in General Practice. Diagnosis related Information from the Monitoring Project. Utrecht: Uitgeversmaatschappij Huisartsenpers b.v.

Lee, D. A., Winslow, N. R., Speight, A. N. P., \& Hey, E. N. (1983). Prevalence and spectrum of asthma in childhood. British Medical Journal, 286, 1256-1258. Levy, M., \& Bell, L. (1984). General practice audit of asthma in childhood.

British Medical Journal, 289, 1115-1116.

Lewis, C. E., Rachelefsky, G., Lewis, M. A., Sota, A., de la, \& Kaplan, M. (1984). A randomized trial of A.C.T. (Asthma Care Training) for kids. Pediatrics, 74. 478.486.

Maiman, L. A., Green, L. W., Gibson, G., \& MacKenzie, E. J. (1979). Education for self-treatment by adult asthmatics. Journal of the American Medical Association, 241, 1919-1922.

Mazzuca, S. A. (1982). Does patient education in chronic diseases have therapeutic value? Journal of Chronic Diseases, 35, 521-529.

McNabb, W. L., Wilson-Pessano, S. R., \& Jacobs, A. M. (1986). Critical selfmanagement competencies for children with asthma. Journal of Pediatric Psychology, 11, 103-117.

McNichol, K. N., Williams, H. E. (1973). Spectrum of asthma in children II, allergic components. British Medical Journal, 4, 12-16.

Mesters, I., Meertens, R., \& Mosterd, N. (1991). Multidisciplinary co-operation in primary care for asthmatic children. Social Science \& Medicine, 32, 65-70. Mesters, I., Pieterse, M., \& Meertens, R. (1991). Pediatric asthma, a qualitative and quantitative approach to needs assessment. Patient Education \& Counseling , 17, 23-34.

Mesters, 1., Meertens. R., Crebolder, H., \& Parcel, G. (1993). Development of a health education program for parents of preschool children with asthma. Health Education Research, 8, 53-68.

Metsemakers, J. F. M., Höppener, P., Knottnerus, J. A., Kocken, R. J. J., \& Limonard, C. B. G. (1992). Computerized health information in the Nether lands: A registration network of family practices. British Journalof General Practice, 42, 102-106.

Mölken, M. P. M. H., van, Doorslaer, E. K. A., \& Rutten, F. F. H. (1989). CARA in cijfers, verslag van een pilot-studie. Maastricht: Institute for Medical Technology Assessment.

Mudde, A., Kok, G., \& Strecher, V. (1989). Self-efficacy and success expectancy as predictors of cessation smoking. Paper presented at the First European Congress of Psychology. Amsterdam. The Netherlands.

Murray, D. M., \& Perry, C. L. (1987). The measurement of substance use 
among adolescents: when is the "bogus pipeline" method needed? Addictive behaviors, 12, 225-233.

National Heart, Lung and Blood Institute, National Institutes of Health (1992).

Definition, diagnosis and classification, the international consensus report on diagnosis and treatment of asthma. European Respiratory Journal, 5, 605-607.

Nationale Kruisvereniging (1988). Jaarrapportage Jeugdgezondheidszorg O-schoolgaand. Kruiswerk 1985 en 1986. Bunnik: Nationale Kruisvereniging.

Newacheck, P. W., Budetti, P. P., \& Halfon, N. (1986). Trends in activitylimiting chronic conditions among children. American Journal of Public Health, 76, 178-184.

Orlandi, M. A., Landers, C., Weston, R., \& Haley, N. (1990). Diffusion of health promotion innovations. In K. Glanz, F. M. Levvis, B. K. Rimeir (Eds.), Healh behavior and health education, theory, research, anol practice (pp. 288-313). San Francisco - Oxford: Jossey-Bass Publishers.

Paulozzi, L. J., Norman, J. E., McMahon, P., \& Connell, F. A. (1984). Outcomes of a diabetes education program. Public Health Reports, 99, 575-579.

Pauwels, R., \& Snashall, P. D. (1986). Astma een Praktische Aanpak. Leusden: CBA Publishing Services/Fisons.

Parcel, G. S., Nader, P. R., \& Tiernan, K. (1980). A health education program for children with asthma. Developmental \& Behavioral Pediatrics, 1, 128-132.

Parcel, G. S., Tiernan, K., Nader, P. R., \& Weiner, L. S. (1984). Teaching myse/f about asthma. Columbia (SC): Health Education Associates.

Parcel, G. S., Taylor, W. C., Brink, S. G., Gottlieb, N., Engquist, K., O'Hara, N. M., \& Eriksen, M. P. (1989). Translating theory into practice: Intervention strategies for the diffusion of a health promotion innovation. Family \& Community Health, 12, 1-13.

Pedersen, S. (1987). Inhaler use in children with asthma. Danish Medical Bulletin, 34, 234-249.

Perry, C. L., Baranowski, T., \& Parcel, G. S. (1990). How individuals, environments, and health behavior interact: Social learning theory. In K. Glanz, F. M. Lewis, \& B. K. Rimer (Eds.), Health behavior and health education, theory, research, and practice (pp. 161-186). San Francisco -Oxford: Jossey-Bass Publishers.

Plaut, T. F. (1988). Children with asthma, a manual for parents. Amherst: Pedipress Inc.

Ploeg, H. M., van der, Defares, P. B., \& Spielberger, C. D. (1980). Handleiding bij de Zelf-Beoordelings vragenlijst, een Nederlandtalige bewerking van de Spielberger State-Trait Anxiety Inventory. Lisse: Swets \& Zeitlinger b.v.

Rachelefsky, G.S. (1987). Review of asthma self-management programs. The Journal of Allergy \& Clinical Immunology, 80, 506-511.

Radius, S. M., Becker, M. H., Rosenstock, I. M., Drachman, R. H., Schuberth, K. C., \& Teets, K. C. (1978). Factors influencing mothers' compliance with a medication regimen for asthmatic children. The Journal of Asthma Research, 15, 133-149. 
Registratienet Huisartspraktijken (1991). Gezondheidsproblemen en

diagnosen in de huisartspraktijk, peiling februari 1991. Maastricht: Rijksuniversiteit Limburg.

Rogers, M. E. (1983). Diffusions of innovations. New York: The Free Press.

Rossi, P. H., \& Freeman, H. E. (1985). Evaluation: a systematic approach.

Beverly Hills: Sage Publications Inc.

Schachter, E. N., Doyle, C. A., \& Beck, G.J. (1984). A prospective study of asthma in a rural community. Chest, 85, 623-630.

Schipper, R. M. (1988). CARA in kaart, samenwerkingsproject van huisartsen en longartsen in Eindhoven. Medisch Contact, 43, 1347-1349.

Schlösser, M. A. G. (1992). Self-management and asthma (thesis). Leiden: DSWO Press.

Schouten, T. J. (1987). De klinische astmascore (Intern verslag). Hilversum: Astmacentrum Heideheuvel

Schouwstra, K. (1976). De continuïteit: "een zorg voor de huisarts". Huisarts \& Wetenschap, 19, 386-390.

Smith, N. A., Seale, J. P., \& Shaw, J. (1984). Medication compliance in children with asthma. Australian Paediatric Journal, 20, 47-51.

Smith, N. A., Seale, J. P., Ley, P., Shaw, J., \& Bracs, P. U. (1986). Effects of intervention on medication compliance in children with asthma. The Medical Journal of Australia, 144, 119-122.

Spector, P. E. (1981). Research designs. Beverly Hills/London: Sage Publications.

Spector, S. L. (1985). Is your asthmatic patient really complying? Annuals Allergy, 55, 552-556.

Speight, A. N. P., Lee, D. A., \& Hey, E. N. (1983). Underdiagnosis and undertreatment of asthma in childhood. British Medical Journal, 286, 1253-1256.

Spykerboer, J. E., Donnelly, W. J., \& Thong, Y. H. (1986). Parental knowledge and misconceptions about asthma, a controlled study. Socia/ Science \& Medicine, $22,553-558$.

Steckler, A., \& Goodman, R. M. (1989). How to institutionalize health promotion programs. American Journal of Health Promotion, 3, 34-44.

Tabachnick, B. G., \& Fidell, L. S. (1989). Using multivariate statistics. New York: Harper \& Row Publishers.

Voorn, T. B. (1983). Chronische ziekten in de huisartspraktijk, een terreinverkenning. Utrecht: Wetenschappelijke Uitgeverij Bunge.

Vries, H., de (1989). Smoking prevention in Dutch schools (Thesis). Maastricht: University of Limburg.

Waart, M. A. C., van der, Gill, K., Dekker, F. W., \& Kaptein, A. A. (1987). Protocollaire benadering van patiënten met astma. The Practitioner, 4, 425-432.

Weiss, J. H. (1981). "Superstuff" self-management educational program for childhood asthma (vols. 2). Bethesda: National Institute of Allergy \& Infectious Disease.

Wever, A. M. J., \& Wever-Hess, J. (1991). Trends in de frequentie van 
ziekenhuisopname wegens astma en chronisch obstructieve longziekte in Nederland, 1980-1988. Nederlands Tijdschrift voor Geneeskunde, 135, 659-664. Wilson-Pessano, S. R., \& Mellins; R. B. (1987). Workshop on asthma self-management, summary of workshop discussion. The Journal of Allergy \& Clinical Immunology, 80, 487-490.

Wigal, J. K., Creer, Th. L., Kotses, H., \& Lewis, P. (1990). A critique of 19 self-management programs for childhood asthma: Part I. The development and evaluation of the programs. Pediatric Asthma, Allergy Immunology, 4, 17-39. 



\section{Summary}

This thesis describes the development, implementation and evaluation of an asthma education protocol in primary health care. Asthma is the most important chronic disease among children. It is known that the majority of the asthma patients already experience symptoms before they are four years of age. Early recognition and adequate treatment of asthma is expected to prevent or reduce fu= ture asthma symptoms. Self-management programs for asthmatic children and their parents have proven to positively contribute to the children's health. However, no program targeted at preschool children was found in literature. Most asthmatic pa= tients are treated in primary care. This finding provides prospects for planned and systematic education in this setting. Therefore, an educational protocol was devel. oped for parents of preschool asthmatic children $(0.4$ years $)$ in primary health care.

In general, the educational protocol aims at identifying the content, the transfer and the organization of the information. The protocol will be developed for use by four disciplines: community nurses, general practitioners, specialized asthma nurses and doctors working at child health centers. The method of the development of the education protocol provides the central subject of this thesis.

Chapter 1 briefly sketches the different planning steps which were taken to develop the education protocol. These steps consisted of two needs assessment studies (among care providers and parents) and a test of the protocol's impact on behavioral variables when it was provided to parents in a group setting. Finally, the protocol was tested in primary care using a randomized controlled trial. One year follow-up data on behavioral variables, process data, medical consumption data and data on asthma severity were collected during a final study. Furthermore, this chapter discusses the theoretical basis for the development of the protocol, including the PRECEDE-model, social learning theory, models for patient-care provider communication and diffusion theory.

Chapter two presents an approach to achieve co-operative education in primary 
care. Because there seemed to be no systematic technique available to divide educational tasks across several disciplines involved, one was developed. The basic idea of this method was that one should link up as far as possible with existing task conceptions and knowledge of the disciplines involved. It was considered important to collect data on whether the discipline in question claimed a certain task, whether the other disciplines agreed with the clain, and whether the discipline in question had appropriate and sufficient knowledge about asthma to perform the claimed task correctly. The developed method seemed to be adequate in assigning tasks to disciplines. Results indicate that the nurses and the general practitioners play the most important roles in asthma care. Each discipline mentioned claimed a considerable number of tasks, and there appears, to be considerable agreement among the disciplines in this respect. Fewer tasks were claimed by and assigned to the doctors of the child health centers. Results fuithermore showed that deficits exist in care providers' knowledge about asthma. It was found that care providers who had once taken a refresher course on asthma had a higher score on the knowledge questionnaire, an indication that the disciplines would profit from more and better training. The above findings imply that the protocol needed to be designed in such a way that health care providers' knowledge on asthma would be improved; the disciplines had to be equipped with the essential knowledge to provide appropriate asthma education.

Chapter three describes a survey focussed on eliciting knowledge, attitudes and misconceptions of parents with asthmatic children. Information about these aspects was gathered by means of a written questionnaire and focus group interviews. The interviews, which allow a free response mode, served as a complementary method to the questionnaire in assessing descriptive information about the target population. Parents of 26 preschool children with asthma were mainly recruited through advertisement in local newspapers. Interviews were conducted with six focus groups. The interviews were directed using a semi-structured interview guide. All sessions were tape recurded. The questionnaire contained some demographic items and questions on knowledge and misconceptions. It was filled out before the group interviews started. The questionnaire provided findings on the extent to which information was known among parents. In general this study revealed that parents know many asthma-related concepts, such as allergy or respiratory infections, but they do not understand well enough what such concepts imply. Several misconceptions about preventive measures, signs preceding an asthma attack and the use of asthma medication were revealed. Results suggest that the care provider who is contacted first and most often for asthma symptoms is the general practitioner; the other disciplines were rarely consulted for asthma. Half the parents indicated in the questionnaire that they vere dissatisfied with the asthma care and education received from their general practitioner. During the interviews even more parents suggested negative experiences in this respect. Considering the small and non-random sample, and an overrepresentation of respondents with a higher education, the results from this study need to be 
interpreted with some care.

In chapter four, the design of the education protocol, based on the results from the two needs assessment surveys, is described. The chapter contains a description of the PRECEDE model applied to preschool asthma. The next part of this chapter presents the results of a pilot study that was conducted to test the protocol in a group setting. General practitioners recruited parents of 35 asthmatic children to participate in a total of three group sessions. The main question of the study was whether the protocol had a positive effect on factors like knowledge, attitude, self-efficacy, self-management behavior and state- anxiety. Data were collected with paper-and-pencil tests. Results indicate that besides a considerable improvement from pre- to post-test in the knowledge and attitude score, there was a notable increase in parents' sense of self-efficacy. Parents' reported selfmanagement behavior changed in the desired direction. In light of the short intervention period of three weeks, parents' reports might better be perceived as an increase in their intention to take appropriate actions rather than a change in their actual performance. Parents' level of state-anxiety wasn't reduced, but this might not be expected after such a short intervention period. The results of this study look promising, but need to be viewed with some caution because a control group was lacking.

In chapter five, the results of a randomized trial with a waiting list control group are reported. The purpose of this study was to test the efficacy of the asthma education protocol in Dutch primary care. A total of 60 general practices (representing 63 general practitioners, 108 patients and (to the patients) related community nurses, asthma nurses and doctors of child health centers) were randomly assigned to either experimental or control group. Process data showed that most health care providers used the protocol according its guidelines and indicated a positive intention to continue using it. Both parents and care providers highly valued the protocol. Mutual referral took place for a small number of children. The number of education modules received by parents was satisfactory. Measuresi of effect were based on the same behavioral variables which were used in the filot study. Pre- to post-test evaluation between both study groups on the behavioral variables suggested that, with the exception of state-anxiety, parents in the experimental group improved significantly compared to the control group. Gain in kriowledge about asthma and gain in the level of self-efficacy were determinants of the improvement in self-management behavior. A final study evaluated the effects of the education protocol at one year follow-up. The main question for the behavioral data was to what extent the behavioral scores, revealed at post-test, were maintained. These data were collected in the experimental group only. Findings indicate that no important changes in the behavioral scores had occurreci. Finally, a major research question focussed on the impact of the protocol on medical care use and asthma severity. Data vvere collected for a 12 -month period preceding and after the intervention period. For this purpose a new land comparable) control group of 38 children was composed as a comparison for the 
remaining 46 children in the experimental group. It was necessary to construct a new control group, as the original waiting list control group had by then received the educationa! protocol too. The data were provided by general practitioners and came from the medica! records. Results indicated that the number of visits to the general practitioners and the number of hospital visits decreased more in the experimental group when compared to the control group. Furthermore, the protocol seems to have a favorable impact on the reduction of asthma severity and duration of symptoms as perceived by the general practitioner. The main conclusion of this thesis is that now an effective asthma education protocol is available for asthmatic children in the 0-4 years age-group. Furthermore the study has given considerable insight into the question of how an educational protocol can be developed in a systematic way. 


\section{Samenvatting}

In dit proefschrift wordt de ontwikkeling, de implementatie in de eerstelijnsgezondheidszorg en de evaluatie beschreven van het voorlichtingsprotocol 'Astma bij kinderen'. Astma is de meest voorkomende chronische ziekte bij kinderen. Bekend is dat de meerderheid van de kinderen symptomen van astma vertoont voor hun vierde levensjaar. Verwacht wordt dat een vroegtijdige signalering en een adequate behandeling van astma de prognose van astmasymptomen gunstig beïnvloeden. Programma's gericht op het leren omgaan met astma door ouders en kinderen. blijken positief bij te dragen aan hun gezondheid. Echter, in de literatuur werd geen programma gevonden dat zich specifiek richt op kinderen in de leeftijd van 0-4 jaar. De meeste kinderen met astma worden behandeld in de eerste lijn. Dit gegeven pleit voor het ontwikkelen van een voorlichtingsprogramma voor deze setting. Resumerend: het onderhavige voorlichtingsprotocol is ontwikkeld voor ouders. waarvan de kinderen (0-4 jaar) worden behandeld in de eerste lijn vanwege astma.

In grote lijnen beschrijft het protocol wie, wat vertelt aan ouders, wanneer en hoe. Het protocol fungeert als hulpmiddel voor hulpverleners, te weten huisartsen, wijkverpleegkundigen, districtsverpleegkundigen-CARA en consultatiebureauartsen, bij het geven van voorlichting.

Centraal in dit proefschrift staat de methode die is toegepast om een voorlichtingsprotocol te ontwikkelen. Hoofdstuk 1 beschrijt in grote lijnen de diverse stappen die zijn doorlopen teneinde het protocol te ontwikkelen en vit te testen. Deze stappen bestaan uit een tweetal behoeftenonderzoeken, eén verricht onder ouders en éen uitgevoerd bij de boven genoemde hulpverleners. Verder is ten behoeve van de ontwikkeling van het protocol een pilotstudie uitgevoerd binnen een aantal patiëntengroepen, waarin de effecten van het protocol werden onderzocht op enkele gedragsvariabelen (kennis, attitude, eigen-effectiviteit, zelfmanagement gedrag en toestandsangst). Uiteindelijk is het protocol ingevoerd in de omgeving waarvoor het werd ontwikkeld, namelijk de eerstelijnsgezondheids- 
zorg. Voor het beoordelen van de effecten van het protocol op de eerder genoemde gedragsvariabelen is een opzet gehanteerd met een willekeurige toewijzing van onderzoekseenheden (randomisatie). De huisartspraktijk is genomen als basis voor de villekeurige toewijzing. Tevens is een procesevaluatie vitgevoerd. Een jaar na de nameting van het gerandomiseerde onderzoek zijn wederom data omtrent de gedragsvariabelen verzameld. Ook zijn gegevens aangaande de procesevaluatie vergaard, tezamen met gegevens over de medische consumptie van patiënten en over de waargenomen ernst van hun astma. Tenslotte wordt in het eerste hoofdstuk kort ingegaan op de onderliggende theoretische principes waarop het protocol is gebaseerd, zoals het PRECEDE-PROCEED model, de sociale leertheorie, modellen voor arts-patiënt communicatie en de diffusietheorie.

In hoofdstuk twee wordt verder ingegaan op het eerste behoeftenonderzoek. Beschreven wordt de gehanteerde benadering voor het verkrijgen van samenwerking betreffende de astmavoorlichting tussen de betrokken hulpverleners. Daar geen systematische methode voorhanden was om voorlichtingstaken tussen diverse disciplines te verdelen is een dergelijke methode uitgewerkt. Het basisidee voor de methode is dat de viteindelijke verdeling zoveel mogelijk dient aan te sluiten bij de bestaande taakopvattingen en kennis over astma van de betrokken hulpverleners. Data zijn verzameld om te achterhalen in hoeverre een discipline in kwestie een bepaalde taak claimt, in hoeverre de overige disciplines het met de betreffende claim eens zịnn en in hoeverre de discipline in kwestie (de eiser) juiste en voldoende kennis heeft om de geclaimde taak naar behoren uit te kunnen voeren. De ontwikkelde methode bleek bruikbaar bij het verdelen van voorlichtingstaken onder diverse disciplines. Uit de resultaten blijkt dat belangrijke rollen zijn weggelegd voor de huisartsen en de wijkverpleegkundigen. Elk van deze twee disciplines claimt diverse taken en krijgt deze ook door de anderen toegewezen. Minder taken zijn opgeëist en toegewezen door de consultatiebureau-artsen en de districtsverpleegkundige-CARA. Deze laatste discipline beschouwt de eigen rol als ondersteunend ten opzichte van de wijkverpleegkundige. Verder duiden de resultaten op het bestaan van kennisgebreken bij hulpverleners ten aanzien van astma. Hulpverleners die ooit een nascholing over astma hebben gevolgd scoren hoger op de kennisvragenlijst; een indicatie voor het nut van meer en adequatere scholing van hulpverleners. De bevindingen suggereren dat het voorlichtingsprotocol dusdanig vorm gegeven dient te worden dat hulpverleners de beschikking krijgen over de essentiële kennis die nodig is voor het geven van goede voorlichting.

Voor een maximaal effect van het voorlichtingsprotocol dient de inhoud zo veel mogelijk aan te sluiten bij de informatie behoeften, interesses, en ideeën van ouders over astma bij kinderen. Als methode om een beeld te verkrijgen van dergelijke gegevens is gebruik gemaakt van het focusgroep interview in combinatie met een schriftelijke vragenlijst. De interviews, die dienen voor het achterhalen van de eerder genoemde gegevens, kunnen worden beschouwd als een complementaire methode ten opzichte van de vragenlijst. Ouders van 26 kinderen, (hoofdzakelijk) geworven via een oproep in huis-aan-huis bladen, zijn verdeeld over zes focusgroe- 
pen. Gedurende de interviews is een semi-gestructureerde vragenlijst gehanteerd als leidraad. Alle interviews zijn op een audioband vastgelegd. De schriftelijke, gestructureerde vragenlijst bevat naast enkele vragen over demografische achtergrondvariabelen, ook kennisvragen (en vragen aangaande misvattingen) over astma en vragen over de ervaringen van ouders met de verkregen astmazorg. De vragenlijst is ingevuld voorafgaande aan de groepsinterviews. Uit dit onderzoek blijkt dat de kennis van ouders onvoldoende is, met name met betrekking tot het medicijngebruik, het ondernemen van acties om astmaklachten te voorkomen en het benoemen van voortekenen van een astma-aanval. Verder blijken ouders bekend te zijn met bepaalde astmatermen, zoals allergie en hyperreactiviteit. Echter, ze weten onvoldoende wat deze begrippen inhouden. Diverse misvattingen die kunnen bijdragen aan het niet of onvoldoende opvolgen van de adviezen van de hulpverleners, zijn gedurende. de groepsdiscussies achterhaald. De huisarts wordt veelal als eerste en het meest frequent geconsulteerd vanwege luchiwegklachten. Ongeveer de helft van de ouders geeft in de vragenlijst te kennen ontevreden te zijn met de behandeling en voorlichting van de huisarts. Gedurende de interviews uit een groter deel van de ouders hun ontevredenheid in deze. Gezien de kleine en niet willekeurig geselecteerde steekproef en een overrepresentatie van ouders met een hogere opleiding, dienen de gevonden resultaten met enige voorzichtigheid te worden betracht. Op basis van de pilotstudie is met name de inhoud van het protocol verder toegesneden op de doelgroep. Hiermee wordt zo veel mogelijk voorkomen dat hulpverleners het gebruik van het voorlichtingsprotocol staken omdat de inhoud van de voorlichting niet aansluit bij de behoeften van hun patiënten.

In hoofdstuk vier wordt beschreven hoe het protocol is vormgegeven, gebaseerd op de twee beschreven behoeftenonderzoeken. Het hoofdstuk bevat een invulling van het PREDEDE-model voor astma bij kinderen. Verder bevat dit hoofdstuk een beschrijving van de pilotstudie waarin de inhoud van het voorlichtingsprotocol gedurende een drietal groepssessies is beoordeeld door 35 ouders. De ouders zijn geselecteerd via huisartsen en vervolgens verdeeld over 6 groepen. Het protocol is door de ouders beoordeeld op begrijpelijkheid en praktische relevantie. Daarnaast is een belangrijk doel het verkrijgen van een eerste indruk van de effecten van het protocol op de gedragsvariabelen kennis, attitude, eigen-effectiviteit, toestandsangst en gerapporteerd zelfmanagement gedrag. Het protocol is geëvalueerd op basis van een design met een voor- en een nameting zonder controlegroep. Uit de resultaten blijken de gemiddelde waarden op de voor-en nameting te verschillen. Ouders rapporteren bij de nameting een toename in kennis en een positievere attitude. Verder maken ouders een positievere inschatting van hun eigen vaardigheid om met astma om te gaan en rapporteren ze een toename in het uitvoeren van zelfmanagement gedrag vergeleken met de voormeting. Gezien de korte interventieperiode van drie weken is de toename in gedrag eerder een indicatie voor het feit dat ouders meer overtuigd zijn van de noodzaak van de uitvoering van bepaalde gedragingen dan van een daadwerkelijke toepassing ervan. De resultaten van de pilotstudie zijn bemoedigend, maar vereisen enige terughou- 
dendheid daar een controlegroep ontbreekt.

In hoofdstuk vijf worden de resultaten beschreven van de gerandomiseerde studie met een wachtlijstcontrolegroep. Het beoogde doel van deze studie is de bestudering van het effect van het protocol bij toepassing ervan in de eerste lijn. In totaal zij̧ 60 praktijken random verdeeld over een experimentele groep en een controlegroep. Binnen deze praktijken werken 63 huisartsen. Deze huisartsen hebben tezamen 108 patiënten geselecteerd. Hierop volgend zijn de wijkverpieegkundigen, de consultatiebureau-artsenendedistrictsverpleegkundigenCARA, behorend bij elk deelnemend patiëntje, benaderd voor deelname.

In de procesevaluatie is nagegaan of en hoe de hulpverleners het programma hebben gebruikt. Tevens is gevraagd naar de gehanteerde methode van overdracht om te achterhalen of de schriftelijke informatie daadwerkelijk is verstrekt aan ouders en in een volgend contact besproken. Uit deze inventarisatie is gebleken dat hulpverleners het protocol gebruiken zoals is voorgeschreven. Verder tonen hulpverleners zich tevreden over het protocol en geven ze aan een positieve intentie te kennen tot continuering van het gebruik van het protocol. Doorverwijzen van patiënten naar andere disciplines vindt plaats op beperkte schaal. De ouders blijken in het bezit te zijn van het merendeel van de informatie over astma. De effecten van het protocol zijn geëvalueerd met dezelfde vragenlijsten als gebruikt voor de pilotstudie. De gevonden verschillen tussen de voor-en nameting suggereren dat, met vitzondering van de variabele toestandsangst, de ouders in de experimentele groep meer veranderen in de gewenste richting dan de ouders in de controlegroep. Toename in kennis en eigen-effectiviteit blijken voorspellers van gerapporteerd zelfmanagement gedrag. In het betreffende hoofdstuk wordt ook de follow-up studie beschreven. Eén jaar na de nameting blijkt de gevonden verandering in de gedragsvariabelen in de experimentele groep behouden te zijn gebleven. Gegevens over de controlegroep ontbreken omdat deze het protocol na de nameting heeft ontvangen, dus derhalve niet meer kan fungeren als controlegroep. Verder zijn medische consumptie data en gegevens over de ernst van astma verzameld bij 28 huisartsen in de experimentele groep. Een nieuwe en vergelijkbare controlegroep is gevormd door 18 huisartsen die zijn aangesloten bij het Registratienetwerk Huisartspraktijken. Patiënten van huisartsen in de experimentele groep vertonen een grotere aframe in het aantal consulten (al dan niet met spoed) aan de huisarts vergeleken met de controlegroep. Verder blijkt het protocol een positief effect te hebben op de afname in ernst van astma en de duur van de astmaklachten zoals waargenomen door de huisartsen.

De hoofdconclusie van dit proefschrift is dat nu een voorlichtingsprotocol beschikbaar is dat speciaal is afgestemd op ouders waarvan de kinderen met astma in de leeftijd van 0-4 jaar worden behandeld in de eerste lijn. Verder bieden de verrichte studies inzicht in de systematische en planmatige wijze, waarop een voorlichtingsprotocol kan worden ontwikkeld. 


\section{Appendix 1}

In the Tables 4, 5 and 6 the opinion of a discipline in question on tasks they consider relevant for their own discipline are presented together with the weighted opinions to this respect of the three remaining disciplines combined. Furthermore, it is indicated per task whether the knovviedge of the discipline in question was sufficient.

In the Tables 7, 8, 9 and 10 the opinions of all four disciplines are presented with respect to the educational tasks of one discipline in question. Here the binomial test of homogeneity was employed whenever the four disciplines did not score all above $\pm 66 \%$ or all below $\pm 66 \%$. Significant deviation in opinion is no problem if the task in question is not assigned to the discipline in question because 1) the discipline in question does not claim the task, or 2) an other discipline is a more likely candidate based on the criteria mentioned in Chapter 2 (reexamine the procedures followed in Table 1, Chapter 2 ). 
Table 4

General practitioners' (GPS) and other disciplines' view on oeneral practitioners' conceptions; and related level of general practitioners' knowledge

\begin{tabular}{|c|c|c|c|c|}
\hline Area & Task & $\begin{array}{l}\text { GP's view } \\
(N=82)\end{array}$ & $\begin{array}{l}\text { View other } \\
\text { disciplines } \\
(\mathbb{N}=157)\end{array}$ & $\begin{array}{c}\text { GP's } \\
\text { knowledge }\end{array}$ \\
\hline $\begin{array}{l}\text { Pointing out } \\
\text { asthma related } \\
\text { problems }\end{array}$ & $\begin{array}{l}\text {-during home visits } \\
\text {-at the child health center } \\
\text {-diagnosing asthma }\end{array}$ & $\begin{array}{l}95.5^{\bullet} \\
10.6^{\circ} \\
98.5^{\bullet}\end{array}$ & $\begin{array}{l}84.0^{\bullet} \\
12.1^{\circ} \\
91.9^{\bullet}\end{array}$ & $\begin{array}{l}\text { Insufficient } \\
\text { Insufficient } \\
\text { Sufficient }\end{array}$ \\
\hline $\begin{array}{l}\text { Pointing out } \\
\text { other problems }\end{array}$ & $\begin{array}{l}\text {-with regard to self care } \\
\text {-with regard to upbringing }\end{array}$ & $\begin{array}{r}100.0^{*} \\
93.9^{*}\end{array}$ & $\begin{array}{l}94.5^{\circ} \\
85.2^{\circ}\end{array}$ & $\begin{array}{l}\text { Not applicable } \\
\text { Not applicable }\end{array}$ \\
\hline Adapting hornes & $\begin{array}{l}\text {-executing home visits } \\
\text {-advice on keeping the } \\
\text { home adapted } \\
\text {-financial cost } \\
\text {-decision }\end{array}$ & $\begin{array}{l}37.9 \\
72.7^{*} \\
29.7 \\
75.8^{*}\end{array}$ & $\begin{array}{l}13.2 \\
53.1 \\
27.1 \\
84.5\end{array}$ & $\begin{array}{l}\text { Insufficient } \\
\text { Insufficient } \\
\text { Sufficient } \\
\text { Not: applicable }\end{array}$ \\
\hline $\begin{array}{l}\text { Providing infor- } \\
\text { mation about } \\
\text { medication }\end{array}$ & $\begin{array}{l}\text {-medication in general } \\
\text {-practice in the use of } \\
\text { medication } \\
\text {-side effects } \\
\text {-appliances } \\
\text {-appropriate use } \\
\text { - peak flow recordings } \\
\text {-monitoring therapy } \\
\text {-follow-up appointment } \\
\text {-during asthma attacks }\end{array}$ & $\begin{array}{l}98.5^{*} \\
77.3^{\star} \\
98.5^{\star} \\
89.2^{\bullet} \\
95.5^{\star} \\
81.8^{\bullet} \\
92.4^{\circ} \\
89.2^{\circ} \\
95.5^{\circ}\end{array}$ & $\begin{array}{r}97.9^{\circ} \\
63.3^{\circ} \\
97.9^{\circ} \\
92.4^{\circ} \\
98.5^{\circ} \\
87.4^{\circ} \\
93.9^{\circ} \\
93.6^{\circ} \\
100.0^{\circ}\end{array}$ & $\begin{array}{l}\text { Sufficient } \\
\text { Insufficient } \\
\text { Sufficient } \\
\text { Sufficient } \\
\text { Sufficient } \\
\text { Insufficient } \\
\text { Sufficient } \\
\text { Not applicable } \\
\text { Not applicable }\end{array}$ \\
\hline $\begin{array}{l}\text { Providing } \\
\text { knowledge } \\
\text { about }\end{array}$ & $\begin{array}{l}\text {-cause of asthma } \\
\text {-prognosis of asthma } \\
\text {-history of asthma } \\
\text { - social consequences } \\
\text {-asthma signals } \\
\text {-asthma triggers } \\
\text {-diagnosis } \\
\text {-consequences }\end{array}$ & $\begin{array}{r}90.9^{\circ} \\
95.4^{*} \\
97.0^{\circ} \\
93.8^{*} \\
100.0^{\circ} \\
97.0^{\circ} \\
100.0^{\circ} \\
98.5^{\circ}\end{array}$ & $\begin{array}{l}96.8^{*} \\
97.0^{*} \\
97.5^{*} \\
79.2^{*} \\
96.4^{*} \\
92.4^{*} \\
96.2^{*} \\
83.8^{*}\end{array}$ & $\begin{array}{l}\text { Sufficient } \\
\text { Sufficient } \\
\text { Insufficient } \\
\text { Insufficient } \\
\text { Insufficient } \\
\text { Insufficient } \\
\text { Sufficient } \\
\text { Not applicable }\end{array}$ \\
\hline $\begin{array}{l}\text { Providing gene- } \\
\text { ral advice about }\end{array}$ & $\begin{array}{l}\text {-physical exercise } \\
\text { - breathing exercise } \\
\text { - behavioral change } \\
\text {-via conservation groups } \\
\text { - imedical) services } \\
\text {-school selection } \\
\text { tools (books etc.) }\end{array}$ & $\begin{array}{c}92.3^{*} \\
73.0^{*} \\
100.0^{*} \\
16.9 \\
68.2^{*} \\
48.4 \\
87.7^{*}\end{array}$ & $\begin{array}{l}84.1^{*} \\
65.3^{*} \\
83.3^{*} \\
14.2^{\circ} \\
51.6 \\
65.7^{\circ} \\
78.0^{\circ}\end{array}$ & $\begin{array}{l}\text { Insufficient } \\
\text { Sufficient } \\
\text { Insufficient } \\
\text { Not applicable } \\
\text { Not applicable } \\
\text { Not applicable } \\
\text { Not: applicable }\end{array}$ \\
\hline $\begin{array}{l}\text { Referring an } \\
\text { asthmatic pa- } \\
\text { tient to a(n) }\end{array}$ & $\begin{array}{l}\text {-specialist } \\
\text {-asthma nurses } \\
\text {-community nurses } \\
\text {-general practitioners } \\
\text {-pedagogue } \\
\text {-physiotherapist }\end{array}$ & $\begin{array}{r}98.5^{\circ} \\
78.8^{\circ} \\
95.5^{\circ} \\
4.5^{\circ} \\
92.4^{*} \\
100.0^{*}\end{array}$ & $\begin{array}{l}99.2^{\circ} \\
57.0^{\circ} \\
98.1^{\circ} \\
3.2^{\circ} \\
97.7^{\circ} \\
98.8^{\circ}\end{array}$ & $\begin{array}{l}\text { Not applicable } \\
\text { Not applicable } \\
\text { Not applicable } \\
\text { Not applicable } \\
\text { Not applicable } \\
\text { Sufficient }\end{array}$ \\
\hline $\begin{array}{l}\text { Coordination of } \\
\text { care }\end{array}$ & $\begin{array}{l}\text { - playing the central role } \\
\text {-being the contact person }\end{array}$ & $\begin{array}{l}89.4^{*} \\
90.9^{*}\end{array}$ & $\begin{array}{l}74.2^{*} \\
81.0^{*}\end{array}$ & $\begin{array}{l}\text { Not applicable } \\
\text { Not: applicable }\end{array}$ \\
\hline $\begin{array}{l}\text { Refresher } \\
\text { course }\end{array}$ & - take a course & $87.9^{*}$ & $87.7^{*}$ & Not applicable \\
\hline
\end{tabular}

Note. - Claim by approximately $2 / 3$ of the respondents of the disciplines involved. 
Table 5

Doctors of child health centers' (DCs) and other disciplines' view on doctors' conceptions, and related level of doctors' knowledge

\begin{tabular}{|c|c|c|c|c|}
\hline Area & Tașk & $\begin{array}{l}\text { DC's view } \\
(N=51)\end{array}$ & $\begin{array}{l}\text { View other } \\
\text { disciplines } \\
(N=188)\end{array}$ & $\begin{array}{c}\text { DC's } \\
\text { Knowledge }\end{array}$ \\
\hline $\begin{array}{l}\text { Pointing out } \\
\text { asthma related } \\
\text { problems }\end{array}$ & $\begin{array}{l}\text {-during home visits } \\
\text {-at the child health center } \\
\text {-diagnosing asthma }\end{array}$ & $\begin{array}{l}8.0 \\
98.0 \\
35.3\end{array}$ & $\begin{array}{c}10.7 \\
100.0^{*} \\
31.1\end{array}$ & $\begin{array}{l}\text { Insufficient } \\
\text { Insufficient } \\
\text { Sufficient }\end{array}$ \\
\hline $\begin{array}{l}\text { Pointing out } \\
\text { other problems }\end{array}$ & $\begin{array}{l}\text {-with regard to self care } \\
\text {-with regard to upbringing }\end{array}$ & $\begin{array}{l}72.5^{*} \\
94.1^{\circ}\end{array}$ & $\begin{array}{l}68.0^{\circ} \\
88.0^{*}\end{array}$ & $\begin{array}{l}\text { Not applicable } \\
\text { Not applicable }\end{array}$ \\
\hline Adapting homes & $\begin{array}{l}\text {-executing home visits } \\
\text {-advice on keeping the } \\
\text { home adapted } \\
\text {-financial cost } \\
\text {-decision }\end{array}$ & $\begin{array}{l}2.0 \\
47.1 \\
11.8 \\
34.0\end{array}$ & $\begin{array}{r}0.9 \\
34.1 \\
9.2 \\
15.9\end{array}$ & $\begin{array}{l}\text { Sufficient } \\
\text { Insufficient } \\
\text { Sufficient } \\
\text { Not applicable }\end{array}$ \\
\hline $\begin{array}{l}\text { Providing infor- } \\
\text { mation about } \\
\text { medication }\end{array}$ & $\begin{array}{l}\text {-medication in general } \\
\text {-practice in the use of } \\
\text { medication } \\
\text {-side effects } \\
\text {-appliances } \\
\text {-individual education about } \\
\text { appropriate use } \\
\text {-peak flow recordings } \\
\text { - monitoring therapy } \\
\text {-follow-up appointment } \\
\text {-during asthma attacks }\end{array}$ & $\begin{array}{r}11.8 \\
2.0 \\
9.8 \\
13.7 \\
\\
5.9 \\
2.0 \\
2.0 \\
2.0 \\
0.0\end{array}$ & $\begin{array}{r}15.5 \\
2.6 \\
10.1 \\
19.9 \\
11.7 \\
4.4 \\
4.3 \\
3.3 \\
0.0\end{array}$ & $\begin{array}{l}\text { Sufficient } \\
\text { Insufficient } \\
\text { Insufficient } \\
\text { Insufficient } \\
\text { Insufficient } \\
\text { Insufficient } \\
\text { Sufficient } \\
\text { Not applicable } \\
\text { Not applicable }\end{array}$ \\
\hline $\begin{array}{l}\text { Providing } \\
\text { knowledge } \\
\text { about }\end{array}$ & $\begin{array}{l}\text {-cause of asthma } \\
\text {-prognosis of asthma } \\
\text {-history of asthma } \\
\text {-social consequences } \\
\text {-asthma signals } \\
\text {-asthma triggers } \\
\text {-diagnosis } \\
\text {-consequences }\end{array}$ & $\begin{array}{r}70.6 \\
56.9 \\
39.2 \\
49.0 \\
56.9 \\
64.7 \\
5.9 \\
51.0\end{array}$ & $\begin{array}{r}43.5 \\
35.2 \\
34.7 \\
34.5 \\
40.9 \\
42.5 \\
5.8 \\
31.7\end{array}$ & $\begin{array}{l}\text { Sufficient } \\
\text { Sufficient } \\
\text { Insufficient } \\
\text { Sufficient } \\
\text { Insufficient } \\
\text { Sufficient } \\
\text { Sufficient } \\
\text { Not applicable }\end{array}$ \\
\hline $\begin{array}{l}\text { Providing gene- } \\
\text { ral advice about }\end{array}$ & $\begin{array}{l}\text {-physical exercise } \\
\text {-breathing exercise } \\
\text {-behavioral change } \\
\text { - via conservation groups } \\
\text {-(medical) services } \\
\text {-school selection } \\
\text {-tools (books etc.) }\end{array}$ & $\begin{array}{l}69.4^{*} \\
20.4^{*} \\
68.0^{*} \\
8.0 \\
30.0 \\
22.9 \\
58.0\end{array}$ & $\begin{array}{r}48.4 \\
22.2 \\
44.6 \\
6.3 \\
22.2 \\
17.8 \\
52.9\end{array}$ & $\begin{array}{l}\text { Sufficient } \\
\text { Sufficient } \\
\text { Insufficient } \\
\text { Not applicable } \\
\text { Not applicable } \\
\text { Not applicable } \\
\text { Not applicable }\end{array}$ \\
\hline $\begin{array}{l}\text { Referring an } \\
\text { asthmatic pa- } \\
\text { tient to a(n) }\end{array}$ & $\begin{array}{l}\text {-specialist } \\
\text {-asthma nurses } \\
\text {-community nurses } \\
\text {-general practitioners } \\
\text {-pedagogue } \\
\text {-physiotherapist }\end{array}$ & $\begin{array}{c}7.8 \\
58.8 \\
82.4^{*} \\
100.0^{*} \\
52.9 \\
13.7\end{array}$ & $\begin{array}{l}10.7 \\
45.9 \\
71.3^{*} \\
98.6^{*} \\
50.3 \\
14.9\end{array}$ & $\begin{array}{l}\text { Not applicable } \\
\text { Not applicable } \\
\text { Not applicable } \\
\text { Not applicable } \\
\text { Not applicable } \\
\text { Sufficient }\end{array}$ \\
\hline $\begin{array}{l}\text { Coordination of } \\
\text { care }\end{array}$ & $\begin{array}{l}\text { playing the central role } \\
\text {-being the contact person }\end{array}$ & $\begin{array}{r}2.0 \\
21.6\end{array}$ & $\begin{array}{r}3.5 \\
10.6\end{array}$ & $\begin{array}{l}\text { Not applicable } \\
\text { Not applicable }\end{array}$ \\
\hline $\begin{array}{l}\text { Refresher } \\
\text { course }\end{array}$ & -take a course & 64.6 & $67.2^{\circ}$ & Not applicable \\
\hline
\end{tabular}

Note. - Claim by approximately $2 / 3$ of the respondents of the disciplines involved. 
Table 6

Asthma nurses' (ANs) and other disciplines' view on asthma nurses' conceptions, and related level of asthma nurses' knowledge

\begin{tabular}{|c|c|c|c|c|}
\hline Area & Task & $\begin{array}{l}\text { AN's view } \\
(N=40)\end{array}$ & $\begin{array}{l}\text { View other } \\
\text { disciplines } \\
(N=199)\end{array}$ & $\begin{array}{c}\text { AN's } \\
\text { Knowledge }\end{array}$ \\
\hline $\begin{array}{l}\text { Pointing out } \\
\text { asthma related } \\
\text { problems }\end{array}$ & $\begin{array}{l}\text {-during home visits } \\
\text {-at the child health center } \\
\text {-diagnosing asthma }\end{array}$ & $\begin{array}{r}46.2 \\
5.0 \\
0.0\end{array}$ & $\begin{array}{r}11.0 \\
1.6 \\
0.9\end{array}$ & $\begin{array}{l}\text { Insufficient } \\
\text { Insufficient } \\
\text { Sufficient }\end{array}$ \\
\hline $\begin{array}{l}\text { Pointing out } \\
\text { other problems }\end{array}$ & $\begin{array}{l}\text {-with regard to self care } \\
\text {-with regard to upbringing }\end{array}$ & $\begin{array}{l}66.7^{\circ} \\
52.5^{\circ}\end{array}$ & $\begin{array}{l}24.2 \\
25.0\end{array}$ & $\begin{array}{l}\text { Not applicable } \\
\text { Not applicable }\end{array}$ \\
\hline $\begin{array}{l}\text { Adapting ho- } \\
\text { mes }\end{array}$ & $\begin{array}{l}\text {-executing home visits } \\
\text {-advice on keeping the } \\
\text { home adapted } \\
\text {-financial cost } \\
\text {-decision }\end{array}$ & $\begin{array}{l}95.0^{*} \\
72.5^{*} \\
90.0^{*} \\
40.0^{*}\end{array}$ & $\begin{array}{l}71.7^{*} \\
59.3^{\circ} \\
72.3^{\circ} \\
42.4^{\circ}\end{array}$ & $\begin{array}{l}\text { Sufficient } \\
\text { Sufficient } \\
\text { Sufficient } \\
\text { Not applicable }\end{array}$ \\
\hline $\begin{array}{l}\text { Providing infor- } \\
\text { mation about } \\
\text { medication }\end{array}$ & $\begin{array}{l}\text {-medication in general } \\
\text {-practice in the use of } \\
\text { medication } \\
\text {-side effects } \\
\text {-appliances } \\
\text {-individual education about } \\
\text { appropriate use } \\
\text {-peak flow recordings } \\
\text {-monitoring therapy } \\
\text {-follow-up appointment } \\
\text {-during asthma attacks }\end{array}$ & $\begin{array}{r}52.5 \\
50.0 \\
15.0 \\
41.0 \\
35.0 \\
10.3 \\
15.4 \\
7.9 \\
0.0\end{array}$ & $\begin{array}{r}19.3 \\
29.4 \\
9.9 \\
23.6 \\
14.5 \\
11.5 \\
6.3 \\
6.0 \\
0.8\end{array}$ & $\begin{array}{l}\text { Sufficient } \\
\text { Sufficient } \\
\text { Insufficient } \\
\text { Sufficient } \\
\text { Insufficient } \\
\text { Insufficient } \\
\text { Insurficient } \\
\text { Not applicable } \\
\text { Not applicable }\end{array}$ \\
\hline $\begin{array}{l}\text { Providing } \\
\text { knowledge } \\
\text { about }\end{array}$ & $\begin{array}{l}\text {-cause of asthma } \\
\text {-prognosis of asthma } \\
\text {-history of asthma } \\
\text {-social consequences } \\
\text {-asthma signals } \\
\text {-asthma triggers } \\
\text {-diagnosis } \\
\text {-consequences }\end{array}$ & $\begin{array}{r}48.7 \\
38.5 \\
43.6 \\
56.4 \\
41.0 \\
53.8 \\
7.7 \\
56.4\end{array}$ & $\begin{array}{r}44.4 \\
33.3 \\
17.9 \\
35.3 \\
28.7 \\
34.9 \\
0.5 \\
42.2\end{array}$ & $\begin{array}{l}\text { Sufficient } \\
\text { Sufficient } \\
\text { Insufficient } \\
\text { Sufficient } \\
\text { Sufficient: } \\
\text { Sufficient } \\
\text { Sufficient } \\
\text { Not applicable }\end{array}$ \\
\hline $\begin{array}{l}\text { Providing } \\
\text { general advice } \\
\text { about }\end{array}$ & $\begin{array}{l}\text {-physical exercise } \\
\text {-breathing exercise } \\
\text {-behavioral change } \\
\text {-via conservation groups } \\
\text {-imedical) services } \\
\text {-school selection } \\
\text {-tools (books etc.) }\end{array}$ & $\begin{array}{l}45.0 \\
17.5 \\
52.5 \\
71.8^{*} \\
75.0^{*} \\
43.6 \\
62.5^{*}\end{array}$ & $\begin{array}{l}32.4 \\
26.3 \\
35.8 \\
65.8 \\
57.7 \\
41.0 \\
47.2\end{array}$ & $\begin{array}{l}\text { Sufficient } \\
\text { Sufficient } \\
\text { Insufficient } \\
\text { Not applicable } \\
\text { Not applicable } \\
\text { Not applicable } \\
\text { Not applicable }\end{array}$ \\
\hline $\begin{array}{l}\text { Referring an } \\
\text { asthmatic pa } \\
\text { tient to a(n) }\end{array}$ & $\begin{array}{l}\text {-specialist } \\
\text {-asthma nurses } \\
\text {-community nurses } \\
\text {-general practitioners } \\
\text {-pedagogue } \\
\text {-physiotherapist }\end{array}$ & $\begin{array}{r}0.0 \\
12.5 \\
87.5 \\
5.0 \\
27.5 \\
2.5\end{array}$ & $\begin{array}{r}0.4 \\
8.6 i \\
34.6 \\
1.4 \\
7.4 \\
1.9\end{array}$ & $\begin{array}{l}\text { Not applicable } \\
\text { Not applicable } \\
\text { Not applicable } \\
\text { Not applicable } \\
\text { Not applicable } \\
\text { Sufficient }\end{array}$ \\
\hline $\begin{array}{l}\text { Coordination } \\
\text { of care }\end{array}$ & $\begin{array}{l}\text {-playing the central role } \\
\text {-being the contact person }\end{array}$ & $\begin{array}{l}33.3 \\
35.9\end{array}$ & $\begin{array}{l}12.0 \\
17.1\end{array}$ & $\begin{array}{l}\text { Not applicable } \\
\text { Not: applicable }\end{array}$ \\
\hline $\begin{array}{l}\text { Refresher } \\
\text { course }\end{array}$ & -take a course & $95.0^{\bullet}$ & $71.5^{\circ}$ & Not applicable \\
\hline
\end{tabular}

Note. Claim by approximately $2 / 3$ of the respondents of the disciplines involved. 
Table 7

Asthma nurses' (ANs), community nurses' (CNs), doctors in child health centers' $(D C s)$ and general practitioners' (GPS) view on general practitioners' tasks

\begin{tabular}{|c|c|c|c|c|c|}
\hline Area & Task & $\begin{array}{c}\text { ANs' } \\
\text { view } \\
(N=40)\end{array}$ & $\begin{array}{c}\text { CNs' } \\
\text { view } \\
(N=82)\end{array}$ & $\begin{array}{c}\text { DCs' } \\
\text { view } \\
(N=51)\end{array}$ & $\begin{array}{c}\text { GPs' } \\
\text { view } \\
(N=66)\end{array}$ \\
\hline $\begin{array}{l}\text { Pointing out } \\
\text { asthma related } \\
\text { problems }\end{array}$ & $\begin{array}{l}\text {-during home visits } \\
\text {-at the child health center } \\
\text {-diagnosing asthma }\end{array}$ & $\begin{array}{r}84.6 \\
0.0 \\
97.5\end{array}$ & $\begin{array}{l}89.0 \\
14.6 \\
93.9\end{array}$ & $\begin{array}{l}80.0 \\
21.6 \\
84.3\end{array}$ & $\begin{array}{l}95.5 \\
10.6 \\
98.5\end{array}$ \\
\hline $\begin{array}{l}\text { Pointing, out } \\
\text { other problems }\end{array}$ & $\begin{array}{l}\text {-with regard to self care } \\
\text {-with regard to upbringing }\end{array}$ & $\begin{array}{l}92.3 \\
87.5\end{array}$ & $\begin{array}{l}96.3 \\
87.8\end{array}$ & $\begin{array}{l}96.1 \\
80.4\end{array}$ & $\begin{array}{r}100.0 \\
93.9\end{array}$ \\
\hline $\begin{array}{l}\text { Adapting } \\
\text { homes }\end{array}$ & $\begin{array}{l}\text {-executing home visits } \\
\text {-advice on keeping the } \\
\text { home adapted } \\
\text {-financial cost } \\
\text {-decision }\end{array}$ & $\begin{array}{r}7.5 \\
45.0 \\
20.0 \\
90.0\end{array}$ & $\begin{array}{r}8.5 \\
\\
47.6 \\
18.3 \\
74.1\end{array}$ & $\begin{array}{l}23.5 \\
66.7 \\
43.1 \\
90.2\end{array}$ & $\begin{array}{l}37.9 \\
72.7^{\bullet} \\
29.7 \\
75.8\end{array}$ \\
\hline $\begin{array}{l}\text { Providing infor- } \\
\text { mation about } \\
\text { medication }\end{array}$ & $\begin{array}{l}\text {-medication in general } \\
\text {-practice in the use of } \\
\text { medication } \\
\text {-side effects } \\
\text {-appliances } \\
\text {-individual education } \\
\text { about appropriate use } \\
\text {-peak flow recordings } \\
\text {-monitoring therapy } \\
\text {-follow-up appointment } \\
\text {-during asthma attacks }\end{array}$ & $\begin{array}{r}97.5 \\
60.0 \\
100.0 \\
94.9 \\
\\
100.0 \\
94.9 \\
89.7 \\
100.0 \\
100.0\end{array}$ & $\begin{array}{r}96.3 \\
67.1 \\
97.6 \\
92.5 \\
97.6 \\
85.0 \\
95.1 \\
93.8 \\
100.0\end{array}$ & $\begin{array}{r}100.0 \\
62.7 \\
96.1 \\
92.2 \\
98.0 \\
84.3 \\
98.0 \\
88.2 \\
100.0\end{array}$ & $\begin{array}{l}98.5 \\
77.3 \\
98.5 \\
89.2 \\
95.5 \\
81.8 \\
92.4 \\
89.2 \\
95.5\end{array}$ \\
\hline $\begin{array}{l}\text { Providing } \\
\text { knowledge } \\
\text { about }\end{array}$ & $\begin{array}{l}\text {-cause of asthma } \\
\text {-prognosis of asthma } \\
\text {-history of asthma } \\
\text {-social consequences } \\
\text {-asthma signals } \\
\text {-asthma triggers } \\
\text {-diagnosis } \\
\text {-consequences }\end{array}$ & $\begin{array}{r}100.0 \\
100.0 \\
100.0 \\
71.8 \\
97.4 \\
87.2 \\
94.9 \\
74.4\end{array}$ & $\begin{array}{r}97.5 \\
100.0 \\
97.5 \\
87.5 \\
95.1 \\
95.1 \\
98.8 \\
84.0\end{array}$ & $\begin{array}{l}94.1 \\
92.2 \\
96.1 \\
80.4 \\
98.0 \\
96.1 \\
96.1 \\
94.1\end{array}$ & $\begin{array}{r}90.9 \\
95.4 \\
97.0 \\
93.8 \\
100.0 \\
97.0 \\
100.0 \\
98.5\end{array}$ \\
\hline $\begin{array}{l}\text { Providing } \\
\text { general advice } \\
\text { abourt }\end{array}$ & $\begin{array}{l}\text {-physical exercise } \\
\text {-breathing exercise } \\
\text {-behavioral change } \\
\text {-via conservation groups, } \\
\text {-(medical) services } \\
\text {-school selection } \\
\text {-tools (books etc.) }\end{array}$ & $\begin{array}{l}80.0 \\
65.0 \\
77.5 \\
15.4 \\
40.0 \\
61.5 \\
82.5\end{array}$ & $\begin{array}{l}85.2 \\
63.8 \\
85.2 \\
13.6 \\
61.3 \\
66.7 \\
74.1\end{array}$ & $\begin{array}{l}91.8 \\
71.4 \\
90.0 \\
14.0 \\
56.0 \\
79.2 \\
80.0\end{array}$ & $\begin{array}{r}92.3 \\
73.0 \\
100.0 \\
16.9 \\
68.2^{*} \\
48.4^{\circ} \\
87.7^{\circ}\end{array}$ \\
\hline $\begin{array}{l}\text { Referring an } \\
\text { asthmatic pa- } \\
\text { tient to a(n) }\end{array}$ & $\begin{array}{l}\text {-specialist } \\
\text {-asthma nurses } \\
\text {-community nurses } \\
\text {-general practitioners } \\
\text {-pedagogue } \\
\text {-physiotherapist }\end{array}$ & $\begin{array}{r}100.0 \\
65.0 \\
100.0 \\
2.5 \\
97.5 \\
97.5\end{array}$ & $\begin{array}{r}97.6 \\
35.4 \\
96.3 \\
6.1 \\
98.8 \\
98.8\end{array}$ & $\begin{array}{r}100.0 \\
70.6 \\
98.0 \\
0.0 \\
98.0 \\
100.0\end{array}$ & $\begin{array}{r}98.5 \\
78.8^{*} \\
95.5 \\
4.5 \\
92.4 \\
100.0\end{array}$ \\
\hline $\begin{array}{l}\text { Coordination } \\
\text { of care. }\end{array}$ & $\begin{array}{l}\text {-playing the central role } \\
\text {-being the contact person }\end{array}$ & $\begin{array}{l}56.4 \\
74.4\end{array}$ & $\begin{array}{l}72.0 \\
75.3\end{array}$ & $\begin{array}{l}94.1 \\
94.1\end{array}$ & $\begin{array}{l}89.4^{\circ} \\
90.9^{\circ}\end{array}$ \\
\hline $\begin{array}{l}\text { Refresher } \\
\text { course }\end{array}$ & -take a course & 92.5 & 82.5 & 95.8 & 87.9 \\
\hline
\end{tabular}

Note. Binomial test of homogeneity; $\rho<.05$. 
Table 8

Asthma nurses' (ANs), community nurses' (CNs), docters in child health centers' (DCs) and general practitioners' (GPs) view on asthma nurses' tasks.

\begin{tabular}{|c|c|c|c|c|c|}
\hline Area & Task & $\begin{array}{c}\text { ANs' } \\
\text { view } \\
(N=40)\end{array}$ & $\begin{array}{c}\mathrm{CNs}^{\prime} \\
\text { view } \\
(N=82)\end{array}$ & $\begin{array}{c}\text { DCs' } \\
\text { view } \\
(N=51)\end{array}$ & $\begin{array}{l}\text { GPs' } \\
\text { view } \\
(N=66)\end{array}$ \\
\hline $\begin{array}{l}\text { Pointing out } \\
\text { asthma related } \\
\text { problems }\end{array}$ & $\begin{array}{l}\text {-during home visits } \\
\text {-at the child health center } \\
\text {-diagnosing asthma }\end{array}$ & $\begin{array}{r}46.2 \\
5.0 \\
0.0\end{array}$ & $\begin{array}{l}7.3 \\
1.2 \\
1.2\end{array}$ & $\begin{array}{r}12.0 \\
2.0 \\
0.0\end{array}$ & $\begin{array}{r}13.6 \\
1.5 \\
1.5\end{array}$ \\
\hline $\begin{array}{l}\text { Pointing out } \\
\text { other problems }\end{array}$ & $\begin{array}{l}\text { - with regard to seif care } \\
\text {-with regard to upbringingl }\end{array}$ & $\begin{array}{l}66.7 \\
52.5\end{array}$ & $\begin{array}{l}24.7 \\
25.6\end{array}$ & $\begin{array}{l}21.6 \\
17.6\end{array}$ & $\begin{array}{l}26.2^{*} \\
31.8\end{array}$ \\
\hline $\begin{array}{l}\text { Adapting } \\
\text { homes }\end{array}$ & $\begin{array}{l}\text {-executing home visits } \\
\text {-advice on keeping the } \\
\text { home adapted } \\
\text {-financial cost } \\
\text {-decision }\end{array}$ & $\begin{array}{l}95.0 \\
72.5 \\
90.0 \\
40.0\end{array}$ & $\begin{array}{l}87.8 \\
72.0 \\
87.8 \\
60.5\end{array}$ & $\begin{array}{l}64.7 \\
52.9 \\
68.6 \\
32.0\end{array}$ & $\begin{array}{l}62.5^{\circ} \\
53.0^{\bullet} \\
60.6^{\circ} \\
34.8^{\circ}\end{array}$ \\
\hline $\begin{array}{l}\text { Providing infor- } \\
\text { mation about } \\
\text { medication }\end{array}$ & $\begin{array}{l}\text {-medication in general } \\
\text {-practice in the use of } \\
\text { medication } \\
\text {-side effects } \\
\text {-appliances } \\
\text {-individual education } \\
\text { about appropriate use } \\
\text {-peak flow recordings } \\
\text {-monitoring therapy } \\
\text {-follow-up appointment } \\
\text {-during asthma attacks }\end{array}$ & $\begin{array}{r}52.5 \\
50.0 \\
15.0 \\
41.0 \\
35.0 \\
10.3 \\
15.4 \\
7.9 \\
0.0\end{array}$ & $\begin{array}{r}17.1 \\
23.2 \\
7.3 \\
30.0 \\
\\
14.6 \\
10.0 \\
2.5 \\
4.9 \\
2.4\end{array}$ & $\begin{array}{l}17.6 \\
25.5 \\
11.8 \\
17.6 \\
7.8 \\
7.8 \\
5.9 \\
3.9 \\
0.0\end{array}$ & $\begin{array}{r}23.1 \\
39.4 \\
10.6 \\
23.1 \\
21.2 \\
16.7 \\
10.6 \\
9.2 \\
0.0\end{array}$ \\
\hline $\begin{array}{l}\text { Providing } \\
\text { knowledge } \\
\text { about }\end{array}$ & $\begin{array}{l}\text {-cause of asthma } \\
\text {-prognosis of asthma } \\
\text {-history of asthma } \\
\text {-social consequences } \\
\text {-asthma signals } \\
\text {-asthma triggers } \\
\text {-diagnosis } \\
\text {-consequences }\end{array}$ & $\begin{array}{r}48.7 \\
38.5 \\
43.6 \\
56.4 \\
41.0 \\
53.8 \\
7.7 \\
56.4\end{array}$ & $\begin{array}{r}38.3 \\
24.7 \\
27.2 \\
32.5 \\
27.2 \\
35.8 \\
0.0 \\
45.7\end{array}$ & $\begin{array}{r}51.0 \\
35.3 \\
9.8 \\
37.3 \\
23.5 \\
31.4 \\
0.0 \\
43.1\end{array}$ & $\begin{array}{r}43.9 \\
40.0 \\
16.7 \\
36.0 \\
35.4 \\
37.9 \\
1.5 \\
37.9\end{array}$ \\
\hline $\begin{array}{l}\text { Providing } \\
\text { general advice } \\
\text { about }\end{array}$ & $\begin{array}{l}\text {-physical exercise } \\
\text {-breathing exercise } \\
\text { - behavioral change } \\
\text { - via conservation groups } \\
\text { - (medical) services } \\
\text {-school selection } \\
\text {-tools. (books etc.) }\end{array}$ & $\begin{array}{l}45.0 \\
17.5 \\
52.5 \\
71.8 \\
75.0 \\
43.6 \\
62.5\end{array}$ & $\begin{array}{l}27.2 \\
17.5 \\
28.4 \\
56.8 \\
52.5 \\
45.3 \\
38.3\end{array}$ & $\begin{array}{l}28.6 \\
12.2 \\
38.0 \\
80.0 \\
66.0 \\
29.2 \\
48.0\end{array}$ & $\begin{array}{l}41.5 \\
31.7 \\
40.9 \\
60.6 \\
5.4 .5 \\
48.4 \\
55.4\end{array}$ \\
\hline $\begin{array}{l}\text { Referring an } \\
\text { asthmatic pa- } \\
\text { tient to a(n) }\end{array}$ & $\begin{array}{l}\text {-specialist } \\
\text {-asthma nurses } \\
\text {-community nurses } \\
\text {-general practitioners } \\
\text {-pedagogue } \\
\text {-physiotherapist }\end{array}$ & $\begin{array}{r}0.0 \\
12.5 \\
87.5 \\
5.0 \\
27.5 \\
2.5\end{array}$ & $\begin{array}{r}1.2 \\
8.5 \\
67.1 \\
1.2 \\
17.3 \\
3.7\end{array}$ & $\begin{array}{r}0.0 \\
9.8 \\
27.5 \\
0.0 \\
2.0 \\
2.0\end{array}$ & $\begin{array}{l}0.0 \\
7.6 \\
9.1 \\
3.0 \\
3.0 \\
0.0\end{array}$ \\
\hline $\begin{array}{l}\text { Coordination } \\
\text { of care }\end{array}$ & $\begin{array}{l}\text {-playing the central role } \\
\text {-being the contact person }\end{array}$ & $\begin{array}{l}33.3 \\
35.9\end{array}$ & $\begin{array}{l}19.5 \\
17.3\end{array}$ & $\begin{array}{r}5.9 \\
15.7\end{array}$ & $\begin{array}{l}10.6 \\
18.2\end{array}$ \\
\hline $\begin{array}{l}\text { Refresher } \\
\text { course }\end{array}$ & -take a course & 95.0 & 81.3 & 77.1 & $56.1^{*}$ \\
\hline
\end{tabular}

Nore. - Binomial test of homogeneity; $\rho<05$. 
Table 9

Asthma nurses' (ANs), community nurses' (CNs), doctors in child health centers' (DCs) and general practitioners' (GPS) view on doctors in child health centers' tasks.

\begin{tabular}{|c|c|c|c|c|c|}
\hline Area & Task & $\begin{array}{c}\text { ANs' } \\
\text { view } \\
(N=40)\end{array}$ & $\begin{array}{c}\text { CNs' } \\
\text { view } \\
(N=82)\end{array}$ & $\begin{array}{c}\text { DCs" } \\
\text { view } \\
(N=51)\end{array}$ & $\begin{array}{c}\text { GPs' } \\
\text { view } \\
(N=66)\end{array}$ \\
\hline $\begin{array}{l}\text { Pointing out } \\
\text { asthma related } \\
\text { problems }\end{array}$ & $\begin{array}{l}\text {-during home visits } \\
\text {-at the child health center } \\
\text {-diagnosing asthma }\end{array}$ & $\begin{array}{r}2.6 \\
100.0 \\
27.5\end{array}$ & $\begin{array}{r}3.7 \\
100.0 \\
35.4\end{array}$ & $\begin{array}{r}8.0 \\
98.0 \\
35.3\end{array}$ & $\begin{array}{r}25.8 \\
100.0 \\
30.3\end{array}$ \\
\hline $\begin{array}{l}\text { Pointing out } \\
\text { other problems }\end{array}$ & $\begin{array}{l}\text {-with regard to self care } \\
\text {-with regard to upbringing }\end{array}$ & $\begin{array}{l}71.8 \\
95.0\end{array}$ & $\begin{array}{l}69.1 \\
90.2\end{array}$ & $\begin{array}{l}72.5 \\
94.1\end{array}$ & $\begin{array}{l}63.1^{\circ} \\
78.8\end{array}$ \\
\hline $\begin{array}{l}\text { Adapting } \\
\text { homes }\end{array}$ & $\begin{array}{l}\text {-executing home visits } \\
\text {-advice on keeping the } \\
\text { home adapted } \\
\text {-financial cost } \\
\text {-decision }\end{array}$ & $\begin{array}{r}0.0 \\
30.0 \\
7.5 \\
10.0\end{array}$ & $\begin{array}{r}1.2 \\
32.9 \\
6.1 \\
21.0\end{array}$ & $\begin{array}{r}2.0 \\
47.1 \\
11.8 \\
34.0\end{array}$ & $\begin{array}{r}1.5 \\
39.4 \\
14.1 \\
16.7\end{array}$ \\
\hline $\begin{array}{l}\text { Providing infor- } \\
\text { mation about } \\
\text { medication }\end{array}$ & $\begin{array}{l}\text {-medication in general } \\
\text {-practice in the use of } \\
\text { medication } \\
\text {-side effects } \\
\text {-appliances } \\
\text {-individual education } \\
\text { about appropriate use } \\
\text {-peak flow recordings } \\
\text { - monitoring therapy } \\
\text {-follow-up appointment } \\
\text {-during asthma attacks }\end{array}$ & $\begin{array}{r}17.5 \\
\\
0.0 \\
10.0 \\
12.8 \\
10.0 \\
0.0 \\
2.6 \\
0.0 \\
0.0\end{array}$ & $\begin{array}{r}18.3 \\
\\
4.9 \\
11.0 \\
23.8 \\
14.6 \\
2.5 \\
7.4 \\
3.7 \\
0.0\end{array}$ & $\begin{array}{r}11.8 \\
\\
2.0 \\
9.8 \\
13.7 \\
5.9 \\
2.0 \\
2.0 \\
2.0 \\
0.0\end{array}$ & $\begin{array}{r}10.8 \\
\\
3.0 \\
9.2 \\
23.1 \\
10.6 \\
10.6 \\
3.0 \\
6.2 \\
0.0\end{array}$ \\
\hline $\begin{array}{l}\text { Providing } \\
\text { knowledge } \\
\text { about }\end{array}$ & $\begin{array}{l}\text {-cause of asthma } \\
\text {-prognosis of asthma } \\
\text {-history of asthma } \\
\text {-social consequences } \\
\text {-asthma signals } \\
\text {-asthma triggers } \\
\text {-diagnosis } \\
\text {-consequences }\end{array}$ & $\begin{array}{r}38.5 \\
28.2 \\
30.8 \\
23.1 \\
35.9 \\
33.3 \\
2.6 \\
20.5\end{array}$ & $\begin{array}{r}55.6 \\
42.0 \\
37.0 \\
45.0 \\
46.9 \\
51.9 \\
8.6 \\
44.4\end{array}$ & $\begin{array}{r}70.6 \\
56.9 \\
39.2 \\
49.0 \\
56.9 \\
64.7 \\
5.9 \\
51.0\end{array}$ & $\begin{array}{l}36.4^{\bullet} \\
35.4 \\
36.4 \\
35.4 \\
40.9 \\
42.4 \\
6.1 \\
30.3\end{array}$ \\
\hline $\begin{array}{l}\text { Providing } \\
\text { general advice } \\
\text { about }\end{array}$ & $\begin{array}{l}\text {-physical exercise } \\
\text {-breathing exercise } \\
\text { - behavioral change } \\
\text { - via conservation groups } \\
\text {-(medical) services } \\
\text {-school selection } \\
\text { - tools (books etc.) }\end{array}$ & $\begin{array}{l}45.0 \\
15.0 \\
40.0 \\
10.3 \\
10.0 \\
15.4 \\
45.0\end{array}$ & $\begin{array}{r}49.4 \\
20.0 \\
38.3 \\
2.5 \\
18.8 \\
14.7 \\
50.6\end{array}$ & $\begin{array}{r}69.4 \\
20.4 \\
68.0 \\
8.0 \\
30.0 \\
22.9 \\
58.0\end{array}$ & $\begin{array}{l}50.8^{*} \\
31.7 \\
56.1^{*} \\
6.2 \\
37.9 \\
23.4 \\
63.1\end{array}$ \\
\hline $\begin{array}{l}\text { Referring an } \\
\text { asthmatic pa- } \\
\text { tient to a(n! }\end{array}$ & $\begin{array}{l}\text {-specialist } \\
\text {-asthma nurses } \\
\text {-community nurses } \\
\text {-general practitioners } \\
\text { - pedagogue } \\
\text {-physiotherapist }\end{array}$ & $\begin{array}{r}10.0 \\
47.5 \\
90.0 \\
100.0 \\
60.0 \\
10.0\end{array}$ & $\begin{array}{l}22.0 \\
40.2 \\
82.9 \\
98.8 \\
66.7 \\
30.5\end{array}$ & $\begin{array}{r}7.8 \\
58.8 \\
82.4 \\
100.0 \\
52.9 \\
13.7\end{array}$ & $\begin{array}{l}0.0 \\
50.0^{*} \\
40.9^{*} \\
97.0^{\circ} \\
24.2^{\circ} \\
4.5\end{array}$ \\
\hline $\begin{array}{l}\text { Coordination } \\
\text { of care }\end{array}$ & $\begin{array}{l}\text {-playing the central role } \\
\text {-being the contact person }\end{array}$ & $\begin{array}{l}2.6 \\
5.1\end{array}$ & $\begin{array}{r}4.9 \\
16.0\end{array}$ & $\begin{array}{r}2.0 \\
21.6\end{array}$ & $\begin{array}{r}3.0 \\
10.6\end{array}$ \\
\hline $\begin{array}{l}\text { Refresher } \\
\text { course }\end{array}$ & -take a course & 67.5 & 68.8 & 64.6 & 65.2 \\
\hline
\end{tabular}

Note. Binomial test of homogeneity: $p<.05$. 
Table 10

Asthma nurses' (ANs), community nurses' (CNis), doctors in child health centers' (DCs) and general practitioners' (GPS) view on community nurses ' tosks.

\begin{tabular}{|c|c|c|c|c|c|}
\hline Area & Task & $\begin{array}{c}\text { ANs' } \\
\text { view } \\
(N=40)\end{array}$ & $\begin{array}{c}\text { CNs' } \\
\text { view } \\
(N=82)\end{array}$ & $\begin{array}{c}D C s^{\prime} \\
\text { view } \\
(N=51)\end{array}$ & $\begin{array}{c}\mathrm{GPS}^{\prime} \\
\text { view } \\
(N=66)\end{array}$ \\
\hline $\begin{array}{l}\text { Pointing out } \\
\text { asthma related } \\
\text { problems }\end{array}$ & $\begin{array}{l}\text {-during home visits } \\
\text {-at the child health center } \\
\text {-diagnosing asthrna }\end{array}$ & $\begin{array}{r}100.0 \\
92.5 \\
0.0\end{array}$ & $\begin{array}{r}98.8 \\
95.1 \\
0.0\end{array}$ & $\begin{array}{r}96.0 \\
70.6 \\
2.0\end{array}$ & $\begin{array}{r}95.5 \\
69.7 \\
4.5\end{array}$ \\
\hline $\begin{array}{l}\text { Pointing out } \\
\text { other problems }\end{array}$ & $\begin{array}{l}\text {-with regard to self care } \\
\text {-with regard to upbringing }\end{array}$ & $\begin{array}{l}100.0 \\
100.0\end{array}$ & $\begin{array}{r}96.3 \\
100.0\end{array}$ & $\begin{array}{l}82.4 \\
98.0\end{array}$ & $\begin{array}{l}81.5 \\
83.3\end{array}$ \\
\hline $\begin{array}{l}\text { Adapting ho- } \\
\text { mes }\end{array}$ & $\begin{array}{l}\text {-executing home visits } \\
\text {-advice on keeping the } \\
\text { home adapted } \\
\text {-financial cost } \\
\text {-decision }\end{array}$ & $\begin{array}{r}100.0 \\
100.0 \\
92.5 \\
40.0\end{array}$ & $\begin{array}{l}97.6 \\
97.6 \\
72.0 \\
53.1\end{array}$ & $\begin{array}{l}92.2 \\
96.1 \\
60.8 \\
34.0\end{array}$ & $\begin{array}{l}77.3 \\
80.3 \\
51.6 \\
40.9\end{array}$ \\
\hline $\begin{array}{l}\text { Providing infor- } \\
\text { mation about } \\
\text { medication }\end{array}$ & $\begin{array}{l}\text {-medication in general } \\
\text {-practice in the use of } \\
\text { medication } \\
\text {-side effects } \\
\text {-appliances } \\
\text {-individiual education } \\
\text { about appropriate use } \\
\text {-peak flow recordings } \\
\text {-monitoring therapy } \\
\text {-follow-up appointment } \\
\text {-during asthma attacks }\end{array}$ & $\begin{array}{r}95.0 \\
100.0 \\
40.0 \\
74.4 \\
10.4 \\
30.8 \\
53.8 \\
44.7 \\
5.0\end{array}$ & $\begin{array}{r}62.2 \\
90.2 \\
28.0 \\
55.0 \\
\\
34.1 \\
12.5 \\
30.9 \\
28.4 \\
3.7\end{array}$ & $\begin{array}{r}27.5 \\
72.5 \\
11.8 \\
39.2 \\
\\
20.4 \\
3.9 \\
9.8 \\
9.8 \\
0.0\end{array}$ & $\begin{array}{c}15.4^{*} \\
63.6^{*} \\
3.1 \\
36.9^{*} \\
12.1 \\
10.6 \\
15.2 \\
9.2 \\
3.0\end{array}$ \\
\hline $\begin{array}{l}\text { Providing } \\
\text { knowledge } \\
\text { about }\end{array}$ & $\begin{array}{l}\text {-cause of asthma } \\
\text {-prognosis of asthma } \\
\text {-history of asthma } \\
\text {-social consequences } \\
\text {-asthma signals } \\
\text {-asthma triggers } \\
\text {-diagnosis } \\
\text {-consequences }\end{array}$ & $\begin{array}{r}79.5 \\
61.5 \\
69.2 \\
94.9 \\
87.2 \\
94.9 \\
5.1 \\
100.0\end{array}$ & $\begin{array}{r}71.6 \\
54.3 \\
53.1 \\
92.5 \\
71.6 \\
86.4 \\
1.2 \\
90.1\end{array}$ & $\begin{array}{r}72.5 \\
52.9 \\
25.5 \\
68.6 \\
68.6 \\
68.6 \\
0.0 \\
68.6\end{array}$ & $\begin{array}{l}45.5^{\star} \\
43.1^{\circ} \\
28.8^{\circ} \\
42.4^{\circ} \\
50.8^{\circ} \\
56.1^{\circ} \\
3.0^{\circ} \\
47.7^{\circ}\end{array}$ \\
\hline $\begin{array}{l}\text { Providing } \\
\text { general advice } \\
\text { about }\end{array}$ & $\begin{array}{l}\text {-physical exercise } \\
\text {-breathing exercise } \\
\text {-behavioral change } \\
\text { - via conservation groups } \\
\text {-(medical) services } \\
\text { - school selection } \\
\text {-tools (books otc.) }\end{array}$ & $\begin{array}{r}90.0 \\
47.5 \\
100.0 \\
89.7 \\
100.0 \\
56.4 \\
100.0\end{array}$ & $\begin{array}{l}77.8 \\
32.5 \\
93.8 \\
86.4 \\
98.8 \\
44.0 \\
98.8\end{array}$ & $\begin{array}{l}63.3 \\
28.6 \\
84.0 \\
60.0 \\
84.0 \\
37.5 \\
98.0\end{array}$ & $\begin{array}{l}49.2^{\bullet} \\
38.1 \\
71.2 \\
53.8^{\circ} \\
71.2 \\
23.4 \\
87.7\end{array}$ \\
\hline $\begin{array}{l}\text { Referring an } \\
\text { asthmatic pa- } \\
\text { tient to } a(n)\end{array}$ & $\begin{array}{l}\text {-specialist } \\
\text {-asthma nurses } \\
\text {-community nurses } \\
\text { - general practitioners } \\
\text {-pedagogue } \\
\text {-physiotherapist }\end{array}$ & $\begin{array}{r}5.0 \\
95.0 \\
17.5 \\
52.5 \\
40.0 \\
5.0\end{array}$ & $\begin{array}{r}4.9 \\
97.6 \\
24.4 \\
52.4 \\
37.0 \\
14.6\end{array}$ & $\begin{array}{r}0.0 \\
80.4 \\
19.6 \\
2.2 .0 \\
13.7 \\
3.9\end{array}$ & $\begin{array}{r}1.5 \\
62.2 \\
61.1 \\
33.3 \\
9.1 \\
1.5\end{array}$ \\
\hline $\begin{array}{l}\text { Coordination } \\
\text { of care }\end{array}$ & $\begin{array}{l}\text {-playing the central role } \\
\text {-being the contact person }\end{array}$ & $\begin{array}{l}61.5 \\
82.1\end{array}$ & $\begin{array}{l}63.4 \\
87.7\end{array}$ & $\begin{array}{r}9.8 \\
43.1\end{array}$ & $\begin{array}{l}16.7^{\circ} \\
36.4^{\circ}\end{array}$ \\
\hline $\begin{array}{l}\text { Refresher } \\
\text { course }\end{array}$ & -take a course & 90.0 & 85.0 & 87.5 & 74.2 \\
\hline
\end{tabular}

Note. Binomial test of homogeneity: $\rho<.05$. 


\section{Appendix 2}

In the asthma education protocol tasks are assigned as follows:

\section{Referral}

The GPs will involve the community nurses whenever an adaptation of a patient's home or training of patient in taking asthma medication is relevant. Furthermgre, GPs are responsible for referring patients to other health care providers such as specialists or physiotherapists. The community nurse is considered responsible for involving the asthma nurse in the care for asthmatic patients. Community nurses and doctors of the health centers will involve one another with respect to their contacts with patients in the child health centers. The pediatric doctor is the main person responsible for involving the GP in care whenever he/she suspects a child has asthma.

\section{Pointing out asthma related problems}

Both GPs and community nurses, will have the task of being mindful of signs indicating asthma while visiting patient's home for other reasons. At the health center the pediatric doctors will also have this task. Confirming the diagnosis is a GPs' task only.

\section{Pointing out general problems}

Both the community nurses and pediatric doctors are responsible for dealing with more general asthma problems such as the raising an asthmatic child and inadequate self care.

\section{Adaptation of homes}

The community and asthma nurses are both relevant disciplines to be involved in the actual execution of an adaptation of homes. In this context the asthma nurse 
will function as support for the community nurse. They are also responsible for explaining the financial consequences of an adaptation to parents. Giving advice on how to keep one's home adapted is a nurses's task as well. However the decision on whether an adaptation should be carried out is one made by the GP.

\section{Coordinator of care}

The GPs turned out to be most acceptable for the role of coordinator of care.

\section{Tasks concerning asthma medication}

The main responsibility for providing education on asthma medication could be assigned to the GP. For instance, education on medication focuses on type, purpose, way of use, side-effects and appliances. Monitoring and follow-up consultations are also includeci. Both the GPs and the nurses have an important role with regard to training parents how to use the medication.

\section{Providing asthma knowledge}

Providing basic asthma information about aetiology and pathogenesis, pathophysiology, symptomatology, precipitants or the rational for self-management actions are initially GPs' and secondly nurses' tasks. The GP is the care provider who will inform parents about the diagnosis and the prognosis. GPs will also discuss the history of the patient. The nurses's tasks is to discuss with parents how asthma can affect family life.

\section{Special points for attention}

For instance, the GPs will take the responsibility to advise parents on their child's participation in physical activities, required behavior change and breathing techniques. Both GPs and community nurses are responsible for providing written materials to people. Because of practical reasons, this task is assigned to GPs. Organizing group meetings for parents and providing information about facilities for asthmatic patient, such as "asthma swimming" is the task of the community and asthma nurses.

Refresher course

All disciplines involved believed that they should take regular refresher courses. 


\section{Appendix 3}

\section{PARENT KNOWLEDGE QUESTIONNAIRE}

Categories: true- ?- false

A. Most children who have os thma:

1) need to be dressed more warmly than children without asthma

2) have both physical and psychological problems

3) can normally take part in any activity requiring some physical exercise

4) cannot go on a holiday

B. When a child has asthma:

5) the heart sometimes doesn't work right

6) the lungs sometimes do not work right

7) the air tubes in the lungs are very sensitive to certain things

8) something is wrong with the child's blood

C. Asthma attacks can be caused by:

9) different things in different people

10) spicy foods like hot peppers and lots of salt

11 things you are allergic to like dust, pollen, animals

12) virus infections like flu

13) irritants (paint, fumes, perfume, smoke, pollution)

14) emotions (laughing too hard, getting upset)

15) drinking too much liquid

16) ternperature difference

17) bacterial infections

18) physical exercise like running

D. Preventing asthma symptoms:

19) is never possible

20) might be possible by removing those things that cause symptoms

21) might be possible by staying away from those things that cause symptoms

22) may be helped by performing warming-up exercises before physical exercise

23) is something only a child's doctor can do anything about

24) may be helped by taking preventive medication 
E. Children with asthms:

25) have less chance of getting symptoms when they are in good physical condition

26) can be cured with medication

27) have a greater chance of keeping symptoms when the severity is worse

28) will remain sensitive

F. When asthmatic children have on attack or asthms symptoms:

29) they have most trouble getting air out of the lungs

30) they have shortness of breath

31) the air tubes inside their lungs become wider

32) the muscles in the air tubes are paralyzed

33) the air passages in the lungs become filled with mucus

34) the tissue of the air tubes gets swollen

35) the muscles in the air tubes go into spasm and tighten

G. Some asthma medication:

36) can be used to prevent an attack or symptoms

37) causes addiction

38) can be used to remedy an attack or symptoms

39) requires increasing dosages

40) causes damaging side effects even in low doses

H. Childron with allorgic asthma:

41) react differently than other people do to the same substance

421 respond especially with their airways

43) only react immediately after contact with triggers

44) have a genetic tendency towards allergy

45) symptoms are triggered by the release of substances in the body after contact with a trigger

I. If chHdren with asthmo ore starting to havo on osthma attack:

46) there is no way one can tell an attack is going to happen

47) one might notice a tight feeling in their chest before the wheezing starts

48) one could give medicine before the wheezing starts

49) one should give medicine only after the wheezing starts

50) the degree to which expiration is prolonged might indicate the severity of the attack

51) they breathe very slowly taking long breaths

52) sucking in the skin of the chest might indicate the severity of the attack (retractions)

53) using a peakflow meter might indicate the severity of the attack

J. When the child has a moderate attack/ symptoms:

54) the parent can do nothing to try and stop the attack

55) the parent must rush to the hospital before doing anything else

56) the parent can give medicine to stop the wheezing

57) it is important the child rests and tries to relax

58) the parent should try not to pay any attention to the wheezing and hope that it will go away

59) the child should drink lots of liquids

60) the parent should call the doctor if breathing does not get better shortly after taking medicine

61) always wait at least a day before calling the doctor to give medicine time to work 
K. Children with hyperreactive airways:

62) react sooner and longer to a substance than other people

63) might get out of breath during and after exercise

64) can trigger an attack by yelling

65) have more symptoms in the early morning due to the low levels of hormones in the blood

L. Adapting the environment focuses on:

66) making the home as dustproof as possible

67) increasing the humidity in the home

68) improving ventilation in the home

69) removing material the child is allergic to

M. When using:

70) a dry-powder inhaler, the powder needs to be inhaled by taking a slow and deep breath

71) an aerosol, the child needs to inhale the spray the moment the spray is released

72) a peakfiow meter, the cursor needs to be moved with a single puff of breath

73) inhalers, the child needs to hold his/her breath for a few seconds after inhalation

\section{ASTHMA ATTITUDE SURVEY}

Categories: strongly agree, agree a little, uncertain, disagree a little, strongly disagree

1. My observations of my child's asthma symptoms are important in helping to get the asthma under control

2. Missing a dose of medication won't hurt

3. Asthma is a problem, oven when my child has no symptoms

4. I consider it important to do things that can prevent my child from getting asthma symptoms

5. My child is like most other kids, except that he/she has asthma

6. I don't believe it is necessary to adapt my home because of my child's asthma

7. It is important to take asthma medicines on time

8. Early detection of asthmai symptoms is important for getting them under control

9. Every asthma attack needs to be treated with medication

10. I think it is important to find out which triggers cause symptoms in my child

11. I have little control over my child's asthma symptoms

12. Eating properly and/or healthy food can help my child's, asthma

13. Taking asthma medication during longer periods of time isn't good for children

14. People with asthma can be successful

15. When taking medicine the adivantages outweigh the disadvantages

16. The more I know about asthma the better I can help my child

17. I don't believe that medicines really help to make symptoms go away 
18. Children with asthma should be disciplined pretty much like other children

19. It is important to keep my child away from triggers

20. Asthma attacks generally do not happen just like that

21. It is important to let my child get used to triggers

22. I consider it important to be able to help my child when she/he has an attack

23. It is no use asking other people to take account of the fact that my child has asthma

24. The fact that asthma symptoms can disappear during puberty is no reason not to treat asthma

\section{PARENT SELF-EFFICACY SURVEY}

Categories: very well, quite well, uncertain, rather poorly, very poorly, not applicable

1. Imagine giving your child medicines: HOW well can you determine the dosage?

2. Imagine your child having a mild or moderate attack: HOW well can you treat this attack?

3. Imagine your child having a severe attack: HOW well can you treat this attack?

4. Imagine your child having asthma symptoms: HOW well can you estimate the moment to start giving medicines?

5. Imagine your child having a mild or moderate attack: HOW well can you estimate the severity of the attack?

6. Imagine your child having a mild or moderate attack: HOW well can you estimate the severity from wheezing?

7. Imagine your child having a mild or moderate attack: HOW well can you estimate the severity from retractions?

8. Inagine your chuld having a mild or moderate attack: HOW well can you estimate the severity from your child's breathing frequency?

9. Imagine your child having a mild or moderate attack: HOW well can you estimate the severity from the relative duration of breathing in and out?

10. Imagine your child having a severe attack: HOW well can you estimate the severity of the attack?

11. Imagine your child having a severe artack: HOW well can you estimate the severity trom wheezing?

12. Imagine your child having a sovere attack:HOW well can you estimate the severity from retractions?

13. Imagine your child having a severe attack: HOW well can you estimate the severity from your child's breathing frequency?

14. Imagine your child having a severe attack: HOW well can you estimate the severity from the relative duration of breathing in and out?

15. HOW well can you avoid asthmal symptoms? 
16. Imagine the symptoms of your child become worse: HOW well can you make the right choice of medicines?

17. Imagine the symptoms of your child become less severe: HOW well can you make the right choice of medicines?

18. Imagine your child having asthma symptoms: HOW well can you judge whether the medication works?

19. HOW well can you indicate what triggers cause asthma problems in your child?

20. HOW well can you decide when to call in the help from others (primary or clinical physician)?

HOW well can you cope with triggers inside the home such as:

21. dust/housemites?

22. animal dander?

23. molds?

24. pollen from trees, grasses and weeds?

25. change in temperature?

26. dampness?

27. cigarette/cigar smoke?

28. smells of detergents, paint, spray or glue?

HOW well can you cope with triggers outside the home such as:

29. fog, rain, humidity?

30. air pollution?

31. HOW well can you influence your child's physical resistance?

32. HOW well can you relax your child during an attack (distracting attention away from attack)?

MANAGEMENT BEHAVIOR SURVEY

Categories: never, seldorn, sometimes, often, always, not applicable

To what extent do you take the following actions to avoid asthma symptoms in your child?

1. Give medication

2. Avoid allergic triggers outside the home

3. Avoid allergic triggers inside the home

4. Avoid chemicals (like smoke, perfumes)

5. Avoid foods my child is sensitive to

6. Ventilate the home correctly

7. Decrease the degree off humidity in the home 
8. Dustproof the home

9. Pay attention to composition of furniture and textiles

10. Stimulate physical activity of my child

To what extent do you take the following actions to treat asthma symptoms in your child?

11. Give medication

12. Give liquids

13. Relax the child

14. Remove triggers

15. Lot the child rest

To what extent do you take the following actions to estimate the severity of an attack?

16. Control breathing frequency

17. Control the duration of breathing in and breathing out

18. Control presence of wheezing

19. Inspect the skin around the chest

20. Use peakfiow meter

\section{STATE-ANXIETY QUESTIONNAIRE}

Categories: not at all, a little, much, very much

When my child has an asthma attack:

1. I foel calm

2. I am tense

3: I feel at ease

4. I feel certain

5. I feel nervous

6. I am indecisive

7. I am relaxed

8. I am worried 
Appendix 4

Means and standard deviations per subscale of the variables attitude, self-efficacy and self. management behavior

\begin{tabular}{|c|c|c|c|c|c|c|c|}
\hline \multirow[t]{2}{*}{ Scale } & \multicolumn{2}{|c|}{ Posttest } & \multicolumn{2}{|c|}{ Follow-up } & \multirow[b]{2}{*}{ t-value } & \multirow[b]{2}{*}{$d f$} & \multirow[b]{2}{*}{$\rho<$} \\
\hline & $M$ & $S O$ & $M$ & So & & & \\
\hline \multicolumn{8}{|l|}{ Attitude: } \\
\hline Prevention & $9.06 i$ & 2.03 & 8.85 & 1.73 & .70 & 28 & .49 \\
\hline Monitoring & 2.44 & 1.29 & 2.68 & 1.23 & -1.28 & 28 & .22 \\
\hline Treatment & 14.41 & 3.34 & 13.86 & 2.89 & 1.02 & 28 & .32 \\
\hline General & 8.72 & 1.18 & 8.71 & 2.05 & .04 & 28 & .97 \\
\hline \multicolumn{8}{|c|}{ Self-efficacy: } \\
\hline Prevention & .92 & .43 & .90 & .33 & .33 & 28 & .75 \\
\hline Monitoring & .87 & .63 & .93 & .40 & -.55 & 27 & .59 \\
\hline Treatment & 1.00 & 40 & 1.15 & .36 & -2.15 & 28 & .04 \\
\hline \multicolumn{8}{|c|}{ Self-management behavior: } \\
\hline Prevention & 3.27 & .41 & 3.15 & .66 & .88 & 28 & .39 \\
\hline Monitoring & 2.59 & .79 & 2.48 & .59 & .69 & 26 & .50 \\
\hline Treatment & 3.36 & .52 & 3.24 & .55 & 1.11 & 27 & .28 \\
\hline
\end{tabular}





\section{Appendix 5}

\section{Asthma clinical scoresheet}

Asthma under the age of 5 years is recognized on purely clinical grounds (National Heart, Lung, and Blood Institute, National Institutes of Health, 1992). The categories for asthma severity were partly derived from the Asthma Clinicai Scoresheet, which was developed in the Netherlands by Schouten (1987) to score children aged 8 . 13 years who were treated for asthma in an asthma clinic. Originally the scores ranged from $01=$ no asthma) to 9 (= very severe asthma). To obtain some uniformity in scoring, some categories were described specifically.

The Asthma Clinical Scoresheet was reduced to 5 categories, as presented below. Furthermore, the description of the clinical features in the categories were simplified to better fit our setting (primary health care) and our age group (children 0-4 years). The underlying score sheet was pretested among three GPs before actual use in the medical consumption study.

\begin{tabular}{|c|c|c|}
\hline Score & Asthma severity & Clinical foatures \\
\hline 0 & No asthma symptoms & \\
\hline 1 & Mild & $\begin{array}{l}\text { - Incidental symptoms, and only mild } \\
\text { symptoms or attacks } \\
\text { - Seldom needs bronchosspasmolytica } \\
\text { - Only difficulties with activity when having asthma } \\
\text { symptoms }\end{array}$ \\
\hline 2 & Between ! en 3 & \\
\hline 3 & Moderate & $\begin{array}{l}\text { - Regularly mild symptoms or mild attacks } \\
\text { - Regularly, but not continuously, need for bron- } \\
\text { chodilators } \\
\text { - Activities during asthma episode are very much } \\
\text { hindereci }\end{array}$ \\
\hline 4 & Between 3 en 5 & \\
\hline 5 & Severe & $\begin{array}{l}\text { - Exacerbations frequent, often severe } \\
\text { - Needs medication (bronchospasmolyticum) daily } \\
\text { - Not able to excerise at all when having symptoms }\end{array}$ \\
\hline
\end{tabular}





\section{Curriculum vitae}

The author of this thesis was born on May 6th 1964 in the Hague. After she received her VWO dipioma in 1982 at the Sint Janscollege in Hoensbroek, she studied Health Education at the University of Limburg. She graduated in September 1986. During the following years she worked as an 'assistent in opleiding' at the Department of Health Education of the University of Limburg on the project "Model development for patient education in the extramural CARA-care". Subsequently, after four years she devoted her time as a researcher for nine months to the project "Long term effects of the educational protocol for children with asthma". The research described in this thesis is related to these two projects. Since January 1992 she has worked at the same department on the project "Dissemination and evaluation of the educational protocol for children with asthma". 
(2)

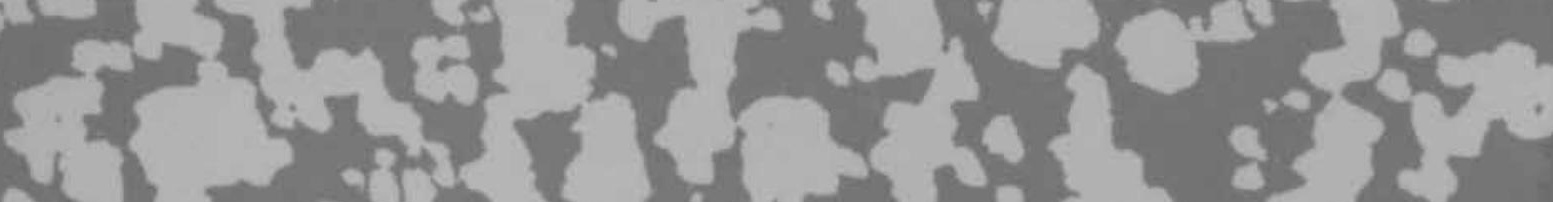

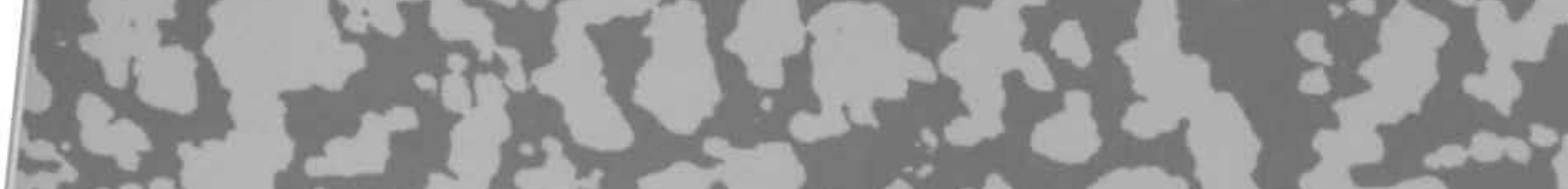

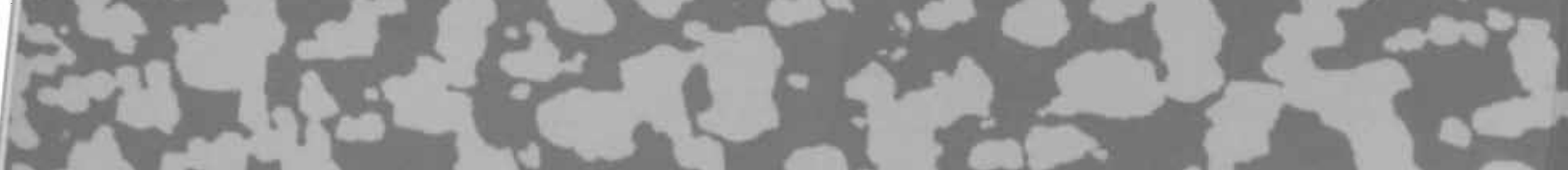
$4 x^{-2}-6$ $\operatorname{loc}^{2} \cot ^{\circ}$

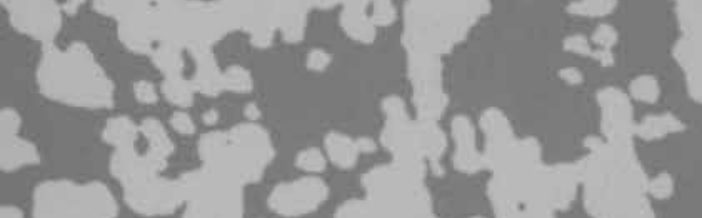
SE

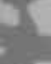
(1) If 